KMU FORSCHUNG AUSTRIA

Austrian Institute for SME Research

TECHNOPOLIS

\title{
tns infratest
}

\section{Begleitende Evaluierung der Kampagne innovatives-oesterreich.at}

Endbericht 
Diese Studie wurde im Auftrag der Plattform bestehend aus BMBWK, BMVIT, BMWA und Rat für Forschung und Technologieentwicklung durchgeführt.

KMU FORSCHUNG AUSTRIA

Austrian Institute for SME Research

(Präsident: em. o. Univ.-Prof. Dr.Dr.h.c. J. Hanns Pichler, M.Sc.)

Für den Inhalt verantwortlich: Walter Bornett

Verfasser/innen des Berichts:

Für die KMU FORSCHUNG AUSTRIA:

Roald Steiner (Projektleitung)

Iris Fischl

Für Technopolis Austria:

Katharina Warta

Leonhard Jörg

Für TNS Infratest Hamburg: Joachim Bacher

Layout:

Martina Gugerell

Die vorliegende Studie wurde nach allen Maßstäben der Sorgfalt erstellt. Die KMU FORSCHUNG AUSTRIA übernimmt jedoch keine Haftung für Schäden oder Folgeschäden die auf diese Studie oder auf mögliche fehlerhafte Angaben zurückgehen.

Dieses Werk ist urheberrechtlich geschützt. Jede Art von Nachdruck, Vervielfältigung, Verbreitung, Wiedergabe, Übersetzung oder Einspeicherung und Verwendung in Datenverarbeitungssystemen, und sei es auch nur auszugsweise, ist nur mit ausdrücklicher Zustimmung der KMU FORSCHUNG AUSTRIA gestattet. 


\section{Inhaltsverzeichnis}

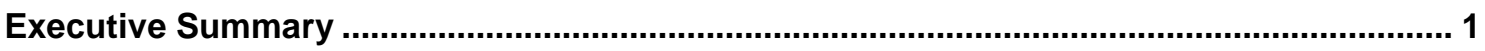

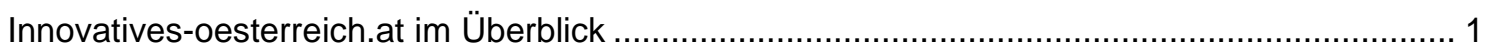

Bestandsaufnahme I: Ergebnisse der Design- und Prozessevaluierung..................................... 1

Bestandsaufnahme II: Ergebnisse der Wirkungsanalyse .................................................... 3

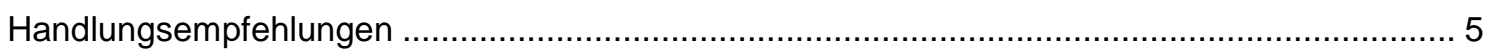

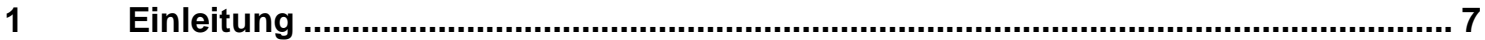

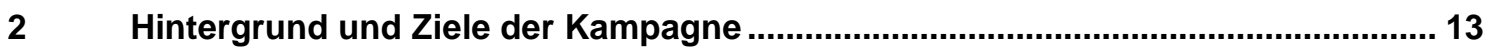

2.1 Awareness-Kampagnen im Bereich Wissenschaft, Forschung und Technologie.......... 13

2.2 Die Kampagne innovatives-oesterreich.at und ihre Mission: Von der

Ratsempfehlung zur Neuorientierung in der 2. Kampagnenphase .............................. 17

3 Strukturen der Kampagne innovatives-oesterreich.at ........................................... 21

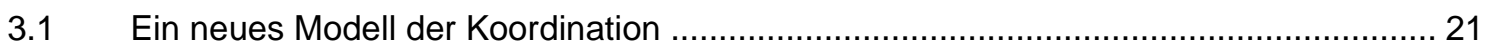

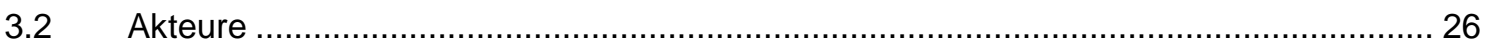

3.3 Projektaktivitäten im Rahmen der zweiten Kampagnenphase von innovatives-

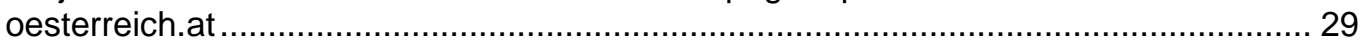

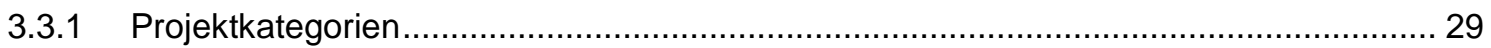

3.3.2 Die einzelnen Projekte - Projekttypen, Zielgruppen und Mobilisierungswege .............. 29

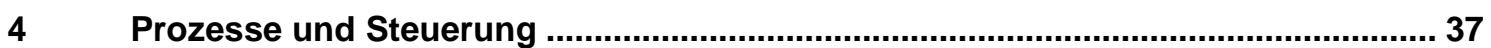

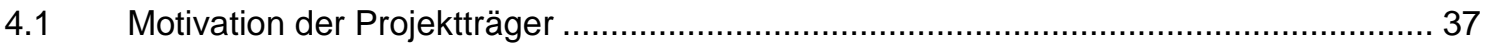

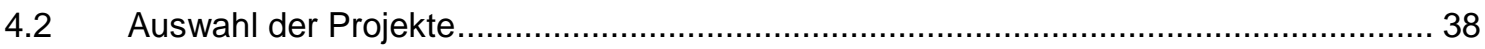

4.3 Programmmanagement - die Sicht der Projektträger .................................................. 39

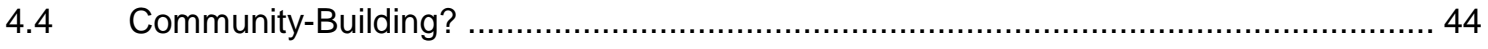

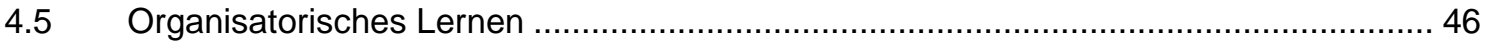

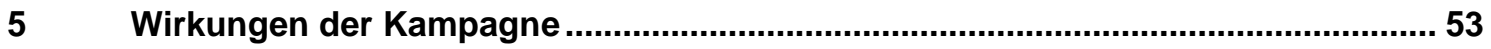

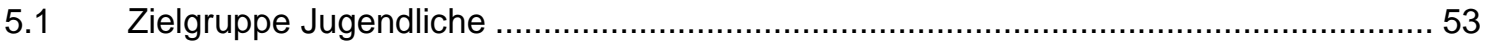

Exkurs: Werbewirkung der Kampagne innovatives-oesterrreich.at im internationalen

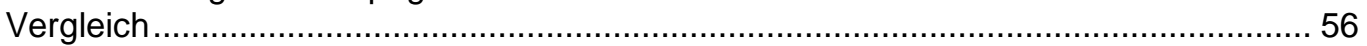

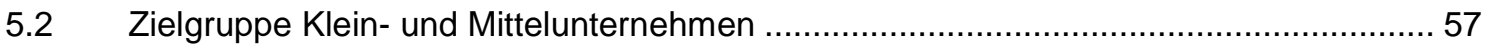

5.3 Das „Vorzeigeprojekt“ Lange Nacht der Forschung 2005 ............................................. 60

5.3.1 Ergebnisse der Besucher/innenbefragung ........................................................... 60

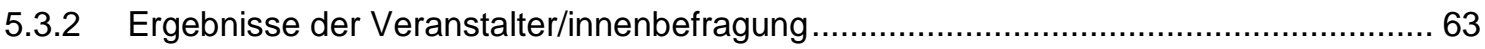

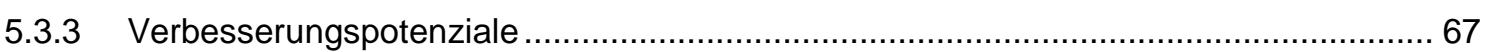

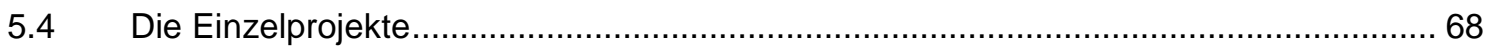


Anhang II: AdEval: Zu Vorgehensweise und Ergebnissen

\section{Tabellenverzeichnis}

Tabelle 1 Lange Nacht der Forschung 2005: Veranstalter/innenbefragung, Rücklauf 10

Tabelle 2 Moderierende Prozessbegleitung im Rahmen der Evaluierung von innovatives-oesterreich.at

Tabelle 3 Projekte im Rahmen der 2. Kampagne innovatives-oesterreich.at ........................ 30

Tabelle 4 Die Entwicklung des organisatorischen Settings und Lerneffekte.

\section{Grafikverzeichnis}

Grafik 1 EU-Umfrage: Auswirkungen von Wissenschaft und Technologie... 14

Grafik 2 Wer erscheint am besten qualifiziert, um die Auswirkungen von Wissenschaft und Technologie zu erklären?

Grafik 3 Struktur der Förderungen im Rahmen von innovatives-oesterreich.at.

Grafik 4 Zuordnung der Kampagnenfunktion „Koordination, Programmmanagement“ zu Kampagnenakteuren.

Grafik 5 Organisation der Kampagne: Mismatch zwischen Beauftragungs- und Koordinationsfunktion

Grafik 6 Zuordnung von Kampagnenfunktionen zu Kampagnenakteuren und Bedeutung der Kampagnenfunktionen - die Sicht der Projektträger/innen

Grafik 7 Projekte im Rahmen der Kampagne innovatives-oesterreich.at nach Projektkategorien ...

Grafik 8

Zielgruppen der Projektaktivitäten

Grafik 9 Publikum der Projektaktivitäten im Rahmen der Kampagne 34

Grafik 10 Reale und potenzielle Mobilisierungswege für Projektaktivitäten im Rahmen der Kampagne....

Grafik 11 Motive für die Teilnahme an der Kampagne.................................................. 37

Grafik 12 Folgeeinreichung von Projekten im Rahmen der Kampagne ............................... 40

Grafik 13 Bewertung der Steuerung und Umsetzung der Kampagne innovativesoesterreich.at

Grafik 14 Beurteilung der PR-Arbeit der Kampagne.

Grafik 15 Bewertung der Bedeutung von Kampagnenaspekten für den unmittelbaren Projekterfolg bzw. für den Kampagnenerfolg

Grafik 16 Wichtige Ansprechpartner für die Durchführung des Projekts/der Projekte ........... 43

Grafik 17 Kommunikationswege mit Programmträgern und -organisatoren der Kampagne . 45

Grafik 18 Gründe der Kenntnis anderer Projekte bzw. Projektträger/innen der Kampagne .. 46 
Grafik 19 Jugendliche: Gestützte Bekanntheit ${ }^{1}$ der Kampagne

innovatives-oesterreich.at (1. Quartal 2006 / 4. Quartal 2006.

Grafik 20 Jugendliche: Gestützte Bekanntheit ${ }^{1}$ des Kampagnen-Logos (Ticket);

1. Quartal 2006 / 4. Quartal 2006

Grafik 21 Jugendliche: Kampagnenbeurteilung global; 1. Quartal 2006 / 4. Quartal 2006 ... 55

Grafik 22 Jugendliche: Kampagnenbeurteilung im Detail

(1. Quartal 2006 / 4. Quartal 2006)....

Grafik 23 Motivationsleistung (Markenimage) und Zielgruppenansprache

(Involvement) der Kampagne innovatives-oesterreich.at, Jugendlichen-Befragung: Jugendliche gesamt.

Grafik 24 Motivationsleistung und Zielgruppenansprache (Involvement) der Kampagne innovatives-oesterreich.at, Jugendlichen-Befragung: Jugendliche nach Geschlecht, Jugendliche nach Bildungsstatus

Grafik 25 KMU: Gestützte Bekanntheit ${ }^{1}$ des Kampagnen-Logos (Ticket) und der Kampagne „innovatives-oesterreich.at“ (1. Quartal 2006 / 4. Quartal 2006).....

Grafik 26 KMU: Gestützte Bekanntheit ${ }^{1}$ des Kampagnen-Logos (Ticket), der Kampagne „innovatives-oesterreich.at“ und der „Langen Nacht der Forschung" (1. Quartal 2006)

Grafik 27 KMU: Kampagnenbeurteilung global (1. Quartal 2006 / 4. Quartal 2006)............. 59

Grafik 28 KMU: Kampagnenbeurteilung im Detail (1. Quartal 2006 / 4. Quartal 2006) ......... 60

Grafik 29 Lange Nacht der Forschung 2005: Motivation der Besucher/innen für den Besuch......

Grafik 30 Lange Nacht der Forschung 2005: Besonderes Interesse der Besucher/innen an wissenschaftlichen Bereichen.

Grafik 31 Lange Nacht der Forschung 2005: Zufriedenheit der Besucher/innen, Global und Detailaspekte.

Grafik 32 Lange Nacht der Forschung 2005: Motive der Veranstalter/innen für die Teilnahme

Grafik 33 Lange Nacht der Forschung 2005: Bewertung der Veranstalter/innen hinsichtlich der Vorbereitung durch die Organisatoren.

Grafik 34 Lange Nacht der Forschung 2005: Erwarteter Nutzen/Effekte der

Teilnahme für die Institutionen aus Sicht der Veranstalter/innen

Grafik 35 Lange Nacht der Forschung 2005: Globalbewertungen durch

Besucher/innen und Veranstalter/innen“.....

Grafik 36 Erwartungen der Besucher bzw. Teilnehmer von Projektaktivitäten -

die Sicht der Projektträger/innen .....

Grafik 37 Erwartete Effekte durch Projektaktivitäten im Rahmen der Kampagne die Sicht der Projektträger/innen 



\section{Executive Summary}

Im Folgenden werden wesentliche Ergebnisse sowie daraus abgeleitete Handlungsempfehlungen der begleitenden Evaluierung der zweiten Phase der Kampagne innovatives-oesterreich.at vorgestellt. Diese wurde im Zeitraum 2005 bis 2006 von der KMU FORSCHUNG AUSTRIA, Technopolis Austria und TNS Infratest Communication Research Centre (Hamburg) durchgeführt. Auftraggeber dieser begleitenden Evaluierung war die Plattform bestehend aus BMBWK, BMVIT, BMWA und Rat für Forschungs- und Technologieentwicklung.

\section{Innovatives-oesterreich.at im Überblick}

(1) Innovatives-oesterreich.at ist eine awareness-Kampagne zur Sensibilisierung einer breiteren Öffentlichkeit für das Themenfeld Wissenschaft, Forschung und Technologie; sie soll zur Verbesserung des wissenschaftspolitischen Klimas beitragen. Derartige Kampagnen sind seit einiger Zeit in zahlreichen OECD-Ländern en vogue. Sie reflektieren einerseits strategische Ziele der Politik und des Wissenschaftssystems, insbesondere im Bereich der Nachwuchs- und Legitimationssicherung, andererseits ein hohes Interesse einer breiteren Öffentlichkeit an Themen aus dem Bereich Wissenschaft, Forschung und Technologie.

(2) Im Fokus der Kampagne innovatives-oesterreich.at stehen dabei zwei Zielgruppen: Zum einen Jugendliche, da beobachtet wird, dass sich diese in Österreich viel zu wenig für den Beruf des/der Forscher/in interessieren, sowie Klein- und Mittelunternehmen, da diese Gruppe mehr als $95 \%$ der österreichischen Unternehmen ausmacht und hier zu aktivierende Innovationspotenziale vermutet werden können.

(3) Im Rahmen der mit 12 Mio. € ausgestatteten zweiten Phase der Kampagne innovativesoesterreich.at (2004-2006) wurden über 50 Projekte durchgeführt. Thematisch reicht das Spektrum der Aktivitäten von der Kinderuniversität über den Erfinderpreis „Jugend Innovativ“, einen Fotowettbewerb, Ausstellungen und Diskussionen bis hin zu dem Großereignis „Lange Nacht der Forschung". Die Spannbreite der Projektbudgets reicht von $18.000 €$ bis hin zu 1,26 Mio $€$. Insgesamt konnten mit den diversen Projektaktivitäten mindestens 267.500 Besucher/innen bzw. Teilnehmer/innen erreicht werden

\section{Bestandsaufnahme I: Ergebnisse der Design- und Prozessevaluierung}

(4) Die Organisation der Plattform innovatives-oesterreich.at stellt in der österreichischen forschungspolitischen Landschaft insofern ein Novum dar, als hier die interministerielle Koordination über einen reinen Informationsaustausch hinausgeht und der installierte Lenkungsausschuss bei zentralen Fragestellungen wie Projektbewilligungen unmittelbare Erntscheidungsbefugnis hat.

(5) Im Vergleich zur ersten Phase der Kampagne innovatives-oesterreich.at (2001-2003) kann für die zweite Phase eine Ausdifferenzierung und Professionalisierung im Programmmanagement beobachtet werden. Hierzu trugen insbesondere die wettbewerbliche Ausschreibung der Kampagne in Modulen sowie die Ausgliederung der Koordinationsfunktionen an spezialisierte Agenturen bei.

(6) Zu Beginn der Evaluierung zeigte sich jedoch rasch, dass das organisationale Setting gravierende "Webfehler" aufwies. Diese betrafen insbesondere die zu schwache bzw. unvollständige Koordination einer derart komplexen Kampagne. Festzustellen waren vor allem eine unzureichende Definition von Schnittstellen unter den beteiligten Akteuren, unklare Aufgabendefinitionen schon bei der Ausschreibung sowie unzureichende vertragliche Fixierungen von Zuständigkeiten. Auf einer grundsätzlichen Ebene herrschte insbesondere Unklarheit, wer auf der einen Seite die Ziele der Kampagne definiert und das Pflichtenheft auflegt, und wer auf der 
anderen Seite die Zielentsprechung garantiert, also Funktionen des Programmmanagements wahrnimmt.

(7) Auf der operativen Ebene machten sich diese strukturellen Defizite als fehlende Steuerungsmöglichkeiten geltend. Es fehlten Pflichtenhefte, verbindliche Milestones und Zeitpläne sowie ein verpflichtendes Berichtswesen. Zudem hat das Nebeneinander von Ressortprojekten und gemeinsamen Projekten zu einem „Vertragschaos“ mit vielen Brüchen geführt, beispielsweise bei der Verwaltung des Finanzausgleichs und bei der Umwidmung von Ressortgeldern für gemeinsame Projekte.

(8) Die Folge war zum einen ein „verronnenes“ Rollenbild der einzelnen Akteure mit entsprechenden Lücken und Doppelgleisigkeiten in den Kommunikations- und Koordinationsprozessen. Zum anderen kam es in diesem Kontext zu Kürzungen von Projekten, unklaren Zielbestimmungen (moving targets), zeitlichen Verzögerungen und insgesamt zu einer reduzierten Kooperationsbereitschaft.

(9) Kommunikations- und Kooperationsschwierigkeiten konnten auf verschiedenen Ebenen beobachtet werden:

- Innerhalb des Lenkungsausschusses gab es unterschiedliche Auffassungen über die konzeptionelle Ausrichtung der Kampagne, die zwischen „klassischen“ Werbemaßnahmen auf der einen und Zielgruppen- und Dialogorientierung auf der anderen Seite schwankten. Zudem erwiesen sich die Entscheidungsfindungsprozesse des Lenkungsausschusses als zu wenig formalisiert und entsprechend nicht hinreichend verbindlich.

- Es gab Unklarheiten bei Projektträger/innen hinsichtlich der Zuständigkeit für die Koordinations- und Öffentlichkeitsarbeit sowie für den „kampagneninternen“ Informationsaustausch und das Community-Building.

- Zwischen den Lead-Agenturen machten sich Reibungen aufgrund ihres wettbewerblichen Verhältnisses zueinander und unterschiedlicher Unternehmenskulturen bemerkbar. Dies hatte Auswirkungen auf die Abgrenzung und Andockung von wahrzunehmenden Aufgabenfeldern: Die Lead-Agenturen mussten mit einem Konkurrenten kooperieren, mit gekürztem Budget arbeiten, und sollten zusätzliche Koordinationsaufgaben wahrnehmen, die so nicht von ihnen geplant waren. Der auch aus diesem Grunde verzögerte Start koordinierter Plattform-Arbeit trug zu einer geringeren Sichtbarkeit der Kampagne bei.

- Zwischen Lead-Agenturen und anderen Projektträger/innen blieb unklar, wer wen unterstützt und inwieweit hier Anforderungen einer wechselseitigen Transparenz bestanden.

- Zwischen Lenkungsausschuss und Lead-Agenturen war nicht hinreichend klar, wer welche Aufgaben definiert und wer für das Programmmanagement zuständig ist.

(10) Eine Folge dieses defizitären organisatorischen Settings war ein erheblicher Mehraufwand durch ungeplante Koordination, der nicht zuletzt zu Lasten der Geschäftsstelle des Rates für FTE ging. Diese war hierfür nicht nur nicht vorgesehen, sondern von den personellen Kapazitäten und Ressourcen her auch nicht ausgestattet. Allerdings war die faktische Übernahme der Koordination durch die Geschäftsstelle des Rates für FTE und das dort entfaltete Aktivitätsniveau ein wesentlicher Faktor der - gemessen an Resonanz und Wirkung - erfolgreichen Weiterführung der Kampagne.

(11) Jenseits der Defizite im organisationalen Setting war zu beobachten, dass die unter dem Dach innovatives-oesterreich.at firmierenden Einzelprojekte offenkundig nicht nur ohne zeitliche Verzögerungen und ohne administrative „Überlastung" durchgeführt werden konnten, sondern dass bei den Projektträger/innen eine "Grundzufriedenheit" hinsichtlich ihrer Teilnahme an der Kampagne vorherrschte; die ganz überwiegende Mehrheit unter ihnen sah ihre Erwartungen 
erfüllt oder gar übertroffen. Dementsprechend hoch ist unter den Projektträger/innen auch das Interesse an Folgeeinreichungen bei einer eventuell anstehenden weiteren Phase der Kampagne innovatives-oesterreich.at

(12) Kritisch sahen die Projektträger/innen das Programmmanagement insofern, als sie eine mitunter als unzureichend empfundene Unterstützung bei Vorbereitung und Durchführung der Projekte monierten. Vermisst wurde insbesondere eine Unterstützung bei der Mobilisierung zu Veranstaltungen; als unzureichend empfunden wurde vielfach auch die kampagnenbezogene PR- und Medienarbeit. Gerade hier wird ein erheblicher Verbesserungsbedarf artikuliert. In diesem Kontext steht auch eine eher kritische Sicht der Projektträger/innen auf die Arbeit der Lead-Agenturen - eine Einschätzung, die allerdings auch im Zusammenhang mit der unklaren Definition der Rolle der Lead-Agenturen und entsprechenden Unklarheiten bei den Projektträger/innen zu sehen sein dürfte. Hierfür spricht, dass die Lead-Agenturen von Seiten der Projektträger/innen auch kaum als relevante Ansprechpartner wahrgenommen wurden.

(13) Der unternommene Versuch des Community-Buildings zielte darauf, die Projektträger/innen mit ihrer "heterogenen Herkunft“ besser unter das Dach der Kampagne zu integrieren, den internen Informationsaustausch zu intensivieren und über eine so angestrebte höhere Kohärenz der Kampagne eine Qualitätssteigerung zu erreichen. Dieses Unterfangen musste allerdings schon deshalb an Grenzen stoßen, da die Projektträger/innen zwar der Kampagne „angehörten“, aber mit Blick auf mögliche Folgeausschreibungen eben auch Wettbewerber mit einem entsprechenden Positionierungsbedarf sind. Dementsprechend kann eigentlich nicht überraschen, dass die Reichweite der eigens zu diesem Zweck organisierten Meet-ups offenkundig begrenzt war und dass auch das als Informationsaustauschplattform installierte technology-log - auch aus anderen Gründen - kaum angenommen wurde.

(14) Die skizzierten organisatorischen Defizite manifestierten sich um den Jahreswechsel 2005/2006 in einer „Steuerungskrise“. Diese Krise wurde zum Anlass für ein deutlich zu beobachtendes „organisatorisches Lernen“, das die Grundlage für eine Stabilisierung wichtiger Kernelemente der Kampagne bildete. Hierzu trug insbesondere die Installation eines Koordinators für begleitende Maßnahmen bei, der weitere Schritte der Öffentlichkeitsarbeit konzipierte und insbesondere dafür Sorge zu tragen hatte, dass die zahlreichen kleineren Projekte über die Sichtbarkeitsschwelle gehoben wurden. Für diese Aktivitäten wurde ein Teil des von den LeadAgenturen verwalteten Budgets zweckgewidmet. Darüber hinaus fand eine Zentralisierung und Straffung der Steuerung statt, indem Lenkungsausschuss und Steuerungsgruppe zusammengezogen wurden und eine zeitliche Abstimmung mit dem Programmkomitee erfolgte.

\section{Bestandsaufnahme II: Ergebnisse der Wirkungsanalyse}

(15) Im Sinne einer dauerhaften Sichtbarkeit entfaltete die Kampagne innovatives-oesterreich.at in ihrer zweiten Phase einen geringen Werbedruck. Angesichts bestehender budgetärer Restriktionen und ihrer auf Zielgruppen und Dialog orientierten Konzeption kann dies nicht überraschen.

(16) Die Kampagne innovatives-oesterreich.at hat die Zielgruppen erreicht: Jedem vierten (Anfang 2006) bzw. fast jedem dritten (Ende 2006) österreichischen Jugendlichen ist die Kampagne innovatives-oesterreich.at bekannt; nahezu ebenso hohe Bekanntheitswerte erreichte auch das Logo. Hinsichtlich der Zielgruppe Klein- und Mittelunternehmen zeigt sich, dass Kampagne und Logo etwa jedem fünften KMU bekannt sind. Die Werbewirkung ist erwartungsgemäß bei höher gebildeten Jugendlichen und „innovationsaffinen“ KMU überdurchschnittlich hoch.

(17) Kampagne und Einzelprojekte adressieren relevante Probleme: Zwischen 50 und $60 \%$ der repräsentativ befragten Jugendlichen sind der Meinung, innovatives-oesterreich.at würde zum Nachdenken anregen, sei ein wichtiger Schritt für die Zukunft Österreichs, motiviere weitere Fragen zu stellen, würde junge Menschen ansprechen und wecke Neugier auf weitere Informationen zu diesem Thema. Jeweils etwa $80 \%$ der befragten KMU geben an, dass aus ihrer Sicht 
die Kampagne innovatives-oesterreich.at zum Nachdenken über Innovationen anregt, junge Menschen anspricht und insgesamt einen wichtigen Schritt für die Zukunft Österreichs darstellt.

(18) Etwa die Hälfte der Jugendlichen und der befragten KMU bewertet die Kampagnenidee mit gut bzw. sehr gut.

(19) Gemessen an vergleichbaren Sensibilisierungskampagnen öffentlicher Auftraggeber, wirkt innovatives-oesterreich.at überdurchschnittlich gut. Wird die von TNS Infratest als Benchmark verwendete Deutschland-Norm herangezogen, so zeigt sich, dass innovatives-oestereich.at eine überdurchschnittlich hohe Zielgruppenansprache (Involvement) aufweist; diese liegt im ersten Quartal 2006 um $+16 \%$ und im vierten Quartal 2006 um $+11 \%$ höher als beim Durchschnitt vergleichbarer Kampagnen. Auch die Motivationsleistung von innovatives-oesterreich.at ist überdurchschnittlich hoch; sie liegt im ersten Quartal 2006 um $+12 \%$ und im vierten Quartal 2006 um $+6 \%$ über dem Durchschnitt vergleichbarer Kampagnen.

(20) Die Lange Nacht der Forschung 2005 kann nicht nur hinsichtlich der budgetären Ausstattung, sondern auch mit Blick auf die Resonanz bei den Besucher/innen und hinsichtlich der Zufriedenheit der Veranstalter/innen als ein „Vorzeigeprojekt“ der Kampagne innovativesoesterreich.at gewertet werden. Unter den Besucher/innen können sich $55 \%$ der Befragten „auf jeden Fall“ einen erneuten Besuch einer Langen Nacht der Forschung vorstellen; nahezu Dreiviertel der befragten Besucher/innen wollen die Lange Nacht „auf jeden Fall“ weiterempfehlen. $32 \%$ der Befragten sehen im Nachhinein ihre Erwartungen an die Lange Nacht der Forschung deutlich übertroffen, mehr als die Hälfte der Veranstalter/innen sieht die Erwartungen als erfüllt an. $56 \%$ der Veranstalter/innen würden im nächsten Jahr „,auf jeden Fall“ nochmals eine Station im Rahmen einer neu aufgelegten Langen der Forschung betreuen. Weitere $28 \%$ der Veranstalter/innen bejahen dies ebenfalls, fügen aber hinzu, dass dies nur unter anderen Bedingungen vorstellbar sei. Hinter diesem letztgenannten Wert verbergen sich noch erhebliche organisatorische Verbesserungspotenziale, insbesondere bei der Vorbereitung, Betreuung und Durchführung einer solchen Veranstaltung.

(21) Die Befragung der Projektträger/innen vermittelt den Eindruck, dass die unter dem Dach von innovatives-oesterreich.at durchgeführten Einzelprojekte die Zielgruppen offenkundig insgesamt gut erreichen; relevante Probleme wurden diesbezüglich auch im Rahmen der qualitativen Erhebungen nicht genannt. Als primäre Effekte durch Projektaktivitäten erwarten sich die Projektträger/innen einen Abbau von Hemmschwellen gegenüber Wissenschaft, Forschung und Technik und die Vermittlung des Nutzens der Forschung in eine breitere Öffentlichkeit.

(22) Zusammenfassend: Gemessen an der auch im internationalen Vergleich überdurchschnittlich hohen Wirkung, hat sich die auf Zielgruppen und Dialog orientierte Konzeption der zweiten Kampagnenphase von innovatives-oesterreich.at bewährt. So ergibt sich insgesamt der Eindruck, dass Thema und Aufbereitung der Kampagne innovatives-oesterreich.at den „Nerv der Zeit" treffen. Die Kampagne und die unter ihrem Dach laufenden Einzelprojekte sind offenkundig weitgehend zielgruppengerecht zugeschnitten. Der Erfolg bzw. die Wirksamkeit der Kampagne innovatives-oesterreich.at ist zudem auch dadurch zu erklären, dass die einzelnen Aktivitäten - ungeachtet aller Schwierigkeiten beispielsweise bei der Verwendung der Dachmarke (Logo) - erkennbar Bestandteil eines Gesamtkonzeptes sind.

(23) Nicht von der Hand zu weisen ist der aus vergleichbaren Kampagnen vertraute Befund, dass auch innovatives-oesterreich.at primär eine ohnehin am Thema Wissenschaft, Forschung und Technologie interessierte und entsprechend sensibilisierte Öffentlichkeit erreicht. Hierfür sprechen sowohl die vorliegenden Informationen zu Bildungsstand und Alter der Besucher/innen von Veranstaltungen, beispielsweise der Langen Nacht der Forschung 2005, sowie die etwas höhere Resonanz bei höher gebildeten Jugendlichen und innovationsaffinen Klein- und Mittelunternehmen. 


\section{Handlungsempfehlungen}

(24) Vor dem Hintergrund der auch im internationalen Vergleich überdurchschnittlich hohen Wirkung der zweiten Kampagne innovatives-oesterreich.at wird empfohlen, das auf Zielgruppen und Dialog orientierte Konzept weiterzuführen.

(25) Die positiven Erfahrungen mit dem Projekt „Lehre und Forschung“, das sich gezielt der Vermittlung von Themen aus dem Bereich Wissenschaft und Forschung an Lehrlinge widmet, sollten zum Anlass genommen werden, die Bemühungen um eher "innovationsindifferente" Adressaten zu intensivieren. Hierfür spricht nicht zuletzt, dass sich auf dem allseits beschworenen Weg in die Wissensgesellschaft der Kreis der in Erwerbs- und Arbeitsleben, aber auch als Staatsbürger/in, in verschiedener Form mit Wissenschaft, Forschung und Technologie „befassten“ Bevölkerungsgruppen kontinuierlich ausweiten sollte.

(26) Die beobachteten Lerneffekte bieten für eine kommende nächste Phase der Kampagne innovatives-oesterreich.at gute Voraussetzungen, um effizientere Strukturen zu finden. Aus Sicht der Evaluation wären dabei folgende Punkte zu berücksichtigen:

- Beibehaltung einer ressortübergreifenden Plattform innovatives-oesterreich.at.

- Klar definierte Aufgaben für eine Lead-Agentur, jedoch getrennte Ausschreibung dieser Funktion. Definition der Funktion als Dienstleister mit vertraglich klar fixierten Aufgaben (Pflichtenheft).

- Durchführung der Ausschreibung der Kampagne sowie der operativen Projektbetreuung durch die FFG.

- Begleitung der Kampagne durch ein gemischt besetztes Komitee, jedoch funktionale Trennung von Konzeption und Programmdesign einerseits und Programmbegleitung andererseits.

- Explizite Aufzählung der Anforderungsprofile und Anforderungen in den Ausschreibungsunterlagen:

- Klare Positionierung hinsichtlich der Mindestanforderungen an die Präsenz im öffentlichen Raum: Projekte und/oder Dachmarke, Hierarchie dieser Aspekte, Zuordnung der Budgets (Dachbudget oder spezifisches Budget für jedes Projekt, aber von der Lead-Agentur verwaltet?).

- Anforderungen an Dachfunktionen: Webpage, Programmmanagement, Community-Building, Pressearbeit, sonstige PR, Coverage der Projekte (nur gemeinsam finanzierte Projekte oder auch Ressortprojekte oder auch Informationsleistung über sonstig finanzierte Projekte in Österreich).

- Anforderungen an die Projektleiter/innen bezüglich Informationsaufbereitung und Berichtlegung.

- Zeitmanagement: Durch allgemein gültige Vorlaufzeiten kommt es zu Projekthäufungen etwa ein Jahr nach der Projektvergabe. Geeignete Maßnahmen, etwa gegenseitige Abstimmung oder entsprechend "gestaffelte" zeitliche Platzierungen (im Rahmen des technisch Möglichen) durch ein Programmmanagement könnten zu einem ausgeglichenen Zeitplan führen.

- Definition der Zielgruppe für PR-Dienstleistungen: Sollen nur kleine Projekte von einer Lead-Agentur in ihrer PR Arbeit unterstützt werden, oder soll es eine 
zentrale PR-Koordination geben, die auch Großprojekte einbindet? Umgekehrt sollten schon in den Ausschreibungsunterlagen die Mindestanforderungen an die Integration der Projekte in eine gemeinsame Kampagne definiert werden, wie beispielsweise die durchgehende Verwendung des gemeinsamen Logos oder die Bereitstellung von Projektunterlagen an die Lead-Agentur für eine koordinierte Pressearbeit. Es war im Zuge der zweiten Kampagne zu beobachten, dass in der Öffentlichkeit die Lange Nacht der Forschung nicht der Kampagne innovatives-oesterreich.at zugeordnet wurde - und das, obwohl die Kampagne unter den Besucher/innen durchaus bekannt war.

(27) Es wird angeraten, im Rahmen der Tätigkeit des Lenkungsausschusses formale Anforderungen (Feststellung der Beschlussfähigkeit, zeitnahe Legung von Protokollen) einzuhalten, damit Entscheidungen des Lenkungsausschusses ein höheres $\mathrm{Maß}$ an Verbindlichkeit zukommt.

(28) Empfohlen wird eine ergebnisorientierte Staffelung der Ausschreibungen und Projektvergabe: Beginnend mit der Einberufung des Programmkomitees und der Definition der Zielsetzungen und Aspekten der neuen Kampagne, anschließend Beauftragung der FFG mit der Ausschreibung dieser Kampagne, dann Ausschreibung der Lead-Agentur, im Weiteren folgend Ausschreibung der gesamten Kampagne, inklusive X.PERIMENTA.

(29) Zusammengefasst: Es gibt Raum für Verbesserungen. Dieser betrifft die Abgrenzung und Definition der Aufgaben Programmdesign, Programmmanagement und Öffentlichkeitsarbeit. Um es zugespitzt zu formulieren: Es wird empfohlen, dabei auf „Innovationen“ zu verzichten und inhaltliche Arbeit ganz traditionell von Management- und Supportleistungen zu trennen. 


\section{$1 \quad$ Einleitung}

Mit dem vorliegenden Bericht legen KMU FORSCHUNG AUSTRIA, Technopolis Austria und TNS Infratest Communication Research Centre (Hamburg) die Ergebnisse der begleitenden Evaluierung der zweiten Phase der Kampagne innovatives-oesterreich.at vor, die im Zeitraum 2005-2006 durchgeführt wurde. Auftraggeber dieser begleitenden Evaluierung war die Plattform bestehend aus BMBWK, BMVIT, BMWA und Rat für Forschungs- und Technologieentwicklung.

\section{Kontext der Evaluierung}

Die Kampagne innovatives-oesterreich.at ist als breitgefächertes Kommunikationsprogramm angelegt und zielt darauf, das Verständnis für den Nutzen von Wissenschaft, Forschung und Technologie zu erhöhen und Aktivitäten in diesem Bereich anzuregen. Sie bündelt eine Reihe von Einzelmaßnahmen und Projekten, die auf den Dialog zwischen Wissenschaft und einer breiteren Öffentlichkeit setzen. Im Fokus der Kampagne stehen dabei zwei Zielgruppen: erstens Jugendliche, da beobachtet wird, dass sich diese in Österreich viel zu wenig für den Beruf des/der Forscher/in interessieren, zweitens Klein- und Mittelunternehmen, da diese Gruppe mehr als $95 \%$ der österreichischen Unternehmen ausmacht und hier zu aktivierende Innovationspotenziale vermutet werden können.

Wie entsprechende Awareness-Kampagnen im Bereich Wissenschaft, Forschung und Technologie in anderen OECD-Ländern zielt auch die Kampagne innovatives-oesterreich.at ${ }^{1}$ auf die Verbesserung des wissenschaftspolitischen Klimas. Sie reflektiert damit zum einen die Einschätzung, die gesellschaftliche Position des Wissenschaftssystems sei angesichts technikkritischer Diskurse gefährdet, zum anderen das Argument, Forschung und technologische Entwicklung seien für Wohlstand und Beschäftigung in wissensbasierten Gesellschaften von existentieller Bedeutung.

\section{Ziele der Evaluierung}

Die begleitende Evaluierung zielt auf die Bereitstellung einer Informationsbasis für die mit der Steuerung der Kampagne befassten Akteure; sie soll Ergebnisse und empirisch informierte handlungsrelevante Hinweise für den Dialog zwischen den Stakeholdern liefern und - im Sinne einer transparenten Bilanz über Erfolge und Defizite - eine Grundlage für allfällige Konsequenzen zur Steuerung und künftigen Gestaltung der Kampagne bereitstellen. Es galt, Reichweite und Resonanz der Kampagne zu erfassen und notwendige Korrekturen herauszuarbeiten, die für eine Steigerung der Qualität bei einer Weiterführung der Kampagne angewendet werden können.

Insofern hat die begleitende Evaluierung vorrangig folgende Funktionen:

1. eine informationsorientierte Lernfunktion für die laufende Arbeit der mit der Steuerung befassten Akteure, aber auch für die Projektträger/innen,

2. eine Lenkungsfunktion für die zukünftige Gestaltung der Öffentlichkeitsarbeit im Bereich der Forschungs- und Technologiepolitik.

Wesentlich ist dabei die Identifizierung von Optimierungshinweisen und von Handlungsoptionen, aber auch von Handlungsnotwendigkeiten während der Kampagnenlaufzeit. Der begleitende Charakter der Evaluierung kommt dabei dadurch zum Ausdruck, dass sie als

Es ist nicht uninteressant zu erwähnen, dass die Wortwahl für das, was unter dem Titel (bzw. der Marke) innovatives-oesterreich.at läuft, nicht einheitlich ist: es wird insbesondere sowohl von einem Dialogprogramm als auch von einer Kampagne gesprochen. Der Ausschreibungstext spricht auch von der Initiative innovativesoesterreich.at. 
interaktiver Prozess zwischen dem Evaluierungsteam, den für die Steuerung verantwortlichen Akteuren und den Durchführenden bzw. Projektträger/innen anzulegen war.

Über die auf Prozessbegleitung zielende Ausrichtung hinaus sollen die Ergebnisse der begleitenden Evaluierung bis zu einem gewissen Grad aber auch stand-alone Gültigkeit haben: Sie soll insofern auch Aussagen ermöglichen, die in der Regel eher einer ex-post Evaluation zuzuordnen sind.

Im Einzelnen war folgenden Fragestellungen nachzugehen:

- Ist das organisatorische Setting zweckmäßig?

- Inwieweit hat sich die Arbeits- und Rollenverteilung (Steuerung, Durchführung) bewährt?

- Wie funktioniert die Zusammenarbeit der involvierten Partner?

- Welche Anreize haben die involvierten Partner sich an der Kampagne zu beteiligen bzw. ihre Ressourcen in den Dienst einer gemeinsamen Zielsetzung einzusetzen?

- Sind die Strukturen und die Prozesse der Kampagne sowie der modulare Aufbau adäquat?

- Ist es bisher gelungen die Balance zwischen Projektvielfalt und nötiger Profilierung zu finden?

- Wie und nach welchen Kriterien werden die Mittel verteilt?

- Haben sich die gewählten Instrumente zur Projektakquisition (Ausschreibung) und selektion bewährt?

- Inwieweit haben die Vorstrukturierung in Module und die Auswahlkriterien die Gesamtkonzeption unterstützt?

- Welche Schwierigkeiten sind im Verlaufe der Kampagne bei den Stakeholdern aufgetreten?

- Existiert ein projektübergreifender Informationsaustausch? Ist/wäre ein solcher sinnvoll?

- Werden die Zielgruppen erreicht, und wenn ja, in welchem Umfang?

- Werden relevante Probleme adressiert?

- Wird/werden die Kampagne und die verschiedenen Instrumente der Kampagne akzeptiert?

- Differiert die Wirkung nach Kampagnentyp und/oder Zielgruppe?

- Welche indirekten Wirkungen gibt es? Sind negative Effekte zu identifizieren?

\section{Vorgehensweise}

Entsprechend des begleitenden Charakters war die Evaluation so anzulegen, dass verschiedene Akteursperspektiven berücksichtigt werden konnten. Insbesondere galt es, in der Diskussion mit den Organisatoren der Kampagne Optionen für Verbesserungen in Ausrichtung und Steuerung der Kampagne auszuloten. Zudem war über eine geeignete Kombination qualitativer und quantitativer Methoden der Evaluationsforschung sicherzustellen, dass im Zuge 
der Evaluation fundierte Ergebnisse und Hinweise zur Steuerung der laufenden Kampagne gegeben werden können.

Um dieser Aufgabenstellung gerecht zu werden, kam ein Methodenmix aus analytisch-bewertenden und moderierend-begleitenden Elementen zum Einsatz. Im Einzelnen:

(1) Aufbauend auf eine umfassende Dokumentenanalyse wurden insgesamt 19 persönliche Interviews mit ehemaligen ${ }^{2}$ und aktuellen Kampagnenakteuren (Stakeholdern) durchgeführt.

(2) Zur Analyse der Wirkungen der Kampagne bei den zwei ausgewählten Zielgruppen Jugendliche und KMU (Kleine- und Mittlere Unternehmen) wurden folgende weitgehend standardisierte Befragungen durchgeführt:

- Methodische Anlage der Jugendlichen-Befragung: Die repräsentative Stichprobe setzt sich aus 400 (1. Befragungswelle) bzw. 403 (2. Befragungswelle) Jugendlichen in Österreich zwischen 14 und 25 Jahren zusammen. ${ }^{3}$ Hierbei wurde auf die Methode der computergestützten Internet-Interviews (CAWI) im Rahmen eines Online-Access-Panels zurückgegriffen.

- Methodische Anlage der KMU-Befragung: Die Stichprobe setzt sich aus 3.913 (1. Befragungswelle) bzw. 4.715 (2. Befragungswelle) österreichischen Gewerbe- und Handwerksbetriebe ${ }^{4}$ zusammen, die im Rahmen der vierteljährlichen Konjunkturbeobachtung der KMU FORSCHUNG AUSTRIA mittels eines standardisierten schriftlichen Fragebogens befragt wurden.

(3) Um auch die Sichtweise und Erfahrungen der Projektträger/innen zu integrieren, wurden neben Interviews mit einzelnen Projektträger/innen - die Projektträger/innen im Zeitraum Ende April bis Anfang Juni 2006 mithilfe eines standardisierten Online-Fragebogens befragt. Von den angeschriebenen 30 Projektträger/innen ${ }^{5}$, haben 28 an der Befragung teilgenommen. Insgesamt deckt die Projektträger-Befragung 37 Projekte im Rahmen von innovatives-oesterreich.at ab. ${ }^{6}$

(4) Im Rahmen der begleitenden Evaluierung wurde auch eine Wirkungsanalyse der ersten Langen Nacht der Forschung in Österreich durchgeführt; dieses „Großereignis“ im Rahmen der zweiten Kampagnenphase fand am 1. Oktober 2005 an den drei Standorten Wien, Linz und Innsbruck statt. Die Wirkungsanalyse basiert auf der Befragung von Besucher/innen und Veranstalter/innen sowie auf den Ergebnissen einer teilnehmenden Beobachtung.

Die Besucher/innenbefragung wurde mittels eines standardisierten zweiseitigen Fragebogens durchgeführt. Dieser Fragebogen wurde im Verlaufe der Langen Nacht der Forschung an allen drei Standorten zum einen von den Veranstalter/innen bzw. den Betreuer/innen der einzelnen Stationen an zentralen Plätzen aufgelegt bzw. verteilt; zum anderen wurde der Fragebogen von fünf Mitarbeiter/innen der KMU FORSCHUNG AUSTRIA entlang ausgewählter „x.peditionen“ verteilt. Auf diese Weise konnte ein Rücklauf an auswertbaren Fragebögen in Höhe von 917 realisiert werden.

2 Akteure der ersten Kampagnenphase von innovatives-oesterreich.at.

3 Die erste Befragungswelle fiel zeitlich in das erste Quartal 2006, die zweite Befragungswelle in das vierte Quartal 2006.

4 Zwar wird dadurch nicht die gesamte Zielgruppe abgebildet, da jedoch knapp $30 \%$ aller österreichischen KMU der Sparte Gewerbe und Handwerk zuzuordnen sind, ist es möglich mit den im Rahmen der Befragung gestellten Zusatzfragen einen guten Hinweis auf die Bekanntheit der Kampagne bei KMU zu geben.

5 Sieben weitere Projektträger/innen wurden nicht befragt, da diese zwar formell der zweiten Kampagnenphase zuzuordnen waren, jedoch ihre Projektaktivitäten bereits vor der Neukonzeption (Ausschreibung des Dialogprogramms) durchgeführt hatten.

$6 \quad$ Mehrere Projekte sind durch ein und denselben Projektträger abgedeckt. 
Die standardisierte Befragung der Veranstalter/innen bzw. von Betreuer/innen von Stationen der Langen Nacht der Forschung erfolgte mithilfe eines Online-Fragebogens. Insgesamt wurden Veranstalter/innen bzw. Betreuer/innen von 151 Stationen angeschrieben. An der Befragung haben 111 Stationen teilgenommen; die Rücklaufquote liegt bei knapp 74 \% (Tabelle 1). Ergänzt wurden die quantitativen Erhebungen durch die teilnehmende Beobachtung, die von fünf Mitarbeiter/innen der KMU FORSCHUNG AUSTRIA an allen drei Standorten durchgeführt wurde.

Tabelle 1 Lange Nacht der Forschung 2005: Veranstalter/innenbefragung, Rücklauf

\begin{tabular}{l|c|c}
\hline Standorte & $\begin{array}{c}\text { Anzahl der angeschriebenen } \\
\text { Stationen }\end{array}$ & Rücklauf (Stationen) \\
\hline Innsbruck & 38 & 24 \\
Linz & 28 & 26 \\
Wien & 85 & 61 \\
\hline \hline Gesamt & $\mathbf{1 5 1}$ & $\mathbf{1 1 1}$ \\
\hline \multicolumn{2}{l}{} \\
\hline
\end{tabular}

(5) Im Rahmen der moderierenden Prozessbegleitung wurden vom Evaluierungsteam während des Untersuchungszeitraums in einer Reihe von Workshops, Diskussionsrunden und Veranstaltungen Teilergebnisse der Evaluierung präsentiert und zur Diskussion gestellt (Tabelle 2). Im Zuge der vom Evaluierungsteam moderierten Gesprächsrunden konnten zusammen mit den beteiligten Akteuren Problembereiche identifiziert und Lösungsmöglichkeiten ausgelotet werden. 
Tabelle 2 Moderierende Prozessbegleitung im Rahmen der Evaluierung von innovativesoesterreich.at

\begin{tabular}{|c|c|c|c|}
\hline Aktivität & Inhalte & Datum & Teilnehmerkreis \\
\hline 1. Workshop & $\begin{array}{l}\text { Präsentation, Reflexion } \\
\text { und Diskussion erster } \\
\text { Ergebnisse der Konzept- } \\
\text { und Prozessbewertung }\end{array}$ & 12. April 2006 & $\begin{array}{ll}\text { - } & \text { Evaluator/innen } \\
\text { - } & \text { Mitglieder des } \\
& \text { Lenkungs- } \\
& \text { Ausschusses }\end{array}$ \\
\hline 2. Workshop & $\begin{array}{l}\text { Präsentation, Reflexion } \\
\text { und Diskussion erster } \\
\text { Ergebnisse der } \\
\text { Wirkungsanalyse }\end{array}$ & 30. Mai 2006 & $\begin{array}{ll}\text { - } & \text { Evaluator/innen } \\
\text { - } & \text { Mitglieder des } \\
& \text { Lenkungs- } \\
& \text { Ausschusses }\end{array}$ \\
\hline 3. Workshop & $\begin{array}{l}\text { Externer Workshop zum } \\
\text { Thema: } \\
\text { „Wirkungen und } \\
\text { Auswirkungen von } \\
\text { Wissenschafts- } \\
\text { kommunikation“ }\end{array}$ & 20.Oktober 2006 & $\begin{array}{ll}\text { - } & \text { Evaluator/innen } \\
\text { - } & \text { Mitglieder des } \\
& \text { Lenkungs- } \\
& \text { Ausschusses } \\
\text { - } & \text { externe Experten } \\
& (\mathrm{D}, \mathrm{CH})^{7} \\
\text { - } & \text { Projektträger }\end{array}$ \\
\hline $\begin{array}{l}\text { Diskussionsrunde mit } \\
\text { Projektträgern }\end{array}$ & $\begin{array}{l}\text { Vertiefende Diskussion } \\
\text { über Erfahrungen und } \\
\text { Einschätzungen mit den } \\
\text { Projektträgern }\end{array}$ & 30. November 2006 & $\begin{array}{l}\text { - Evaluator/innen } \\
\text { - Lead-Agenturen } \\
\text { - } \text { Medienkoordinator } \\
\text { - einzelne } \\
\text { Projektträger }\end{array}$ \\
\hline $\begin{array}{l}\text { Abschluss- } \\
\text { Veranstaltung der } \\
\text { Kampagne II }\end{array}$ & $\begin{array}{l}\text { Rück- und Ausblick für } \\
\text { die Kampagne II } \\
\text { - Präsentation } \\
\text { ausgewählter } \\
\text { Projekte } \\
\text { - Präsentation } \\
\text { Evaluierungs- } \\
\text { ergebnisse } \\
\text { - Diskussion }\end{array}$ & 4. Dezember 2006 & $\begin{array}{l}\text { - } \quad \text { Evaluator/innen } \\
\text { - } \text { Mitglieder des } \\
\text { Lenkungs- } \\
\text { Ausschusses } \\
\text { - } \text { Projektträger } \\
\text { - } \quad \text { Medien- } \\
\text { vertreter/innen }\end{array}$ \\
\hline $\begin{array}{l}\text { Sitzung des } \\
\text { Programmkomitees }\end{array}$ & $\begin{array}{l}\text { Präsentation: } \\
\text { „Wahrnehmung und } \\
\text { Wirkung der Kampagne“; } \\
\text { „Strukturen und } \\
\text { Organisation - Lehren für } \\
\text { die nächste Runde“ } \\
\text { Diskussion }\end{array}$ & 22. Jänner 2007 & $\begin{array}{l}\text { - Evaluator/innen } \\
\text { - } \quad \text { Programmkomitee }\end{array}$ \\
\hline $\begin{array}{l}\text { Sitzung des Rates für } \\
\text { FTE }\end{array}$ & $\begin{array}{l}\text { Präsentation der } \\
\text { Ergebnisse der } \\
\text { Evaluierung vor dem Rat } \\
\text { für FTE }\end{array}$ & $\begin{array}{l}\text { geplanter Termin: } \\
\text { 14. März } 2007\end{array}$ & $\begin{array}{l}\text { - } \\
\text { - } \\
\text { Mitglieder des } \\
\text { Rates für FTE }\end{array}$ \\
\hline
\end{tabular}

Vorträge von Prof. Kurt Imhof (Universität Zürich) und Dirk Strubberg (Pleon Financial Communications). 


\section{Berichtsaufbau}

Entsprechend der skizzierten Aufgabenstellung gliedert sich der vorliegende Bericht wie folgt:

Zunächst werden Hintergrund, „Mission“ und Zielsetzung der Kampagne innovativesoesterreich.at dargestellt (Kapitel 2). Unter Rückgriff auf die einschlägige Diskussion um Wissenschaftskommunikation und die Kampagnenforschung werden wesentliche Aspekte skizziert, die bei der Bewertung einer solchen Kampagne zu berücksichtigen sind (2.1). Hier anschließend wird zum besseren Verständnis der Besonderheiten der zweiten Phase der Kampagne innovatives-oesterreich.at die Vorgeschichte skizziert (2.2).

Gegenstand des 3. Kapitels sind die Strukturen und das organisatorische Setting der Kampagne. Von Bedeutung ist hier zunächst das neue Modell der Koordination (3.1), das auch das Handeln der beteiligten Akteure wesentlich beeinflusst (3.2) und die Strukturen der im Rahmen der Kampagne durchgeführten Projekte geprägt hat (3.3).

Im Weiteren werden die Ergebnisse der Prozessevaluierung vorgestellt (Kapitel 4). Im Vordergrund steht zunächst die Motivation der Projektträger/innen zur Teilnahme an der Kampagne, da diese für den Erfolg der Kampagne sowie für die im Rahmen von innovatives-oesterreich.at ablaufenden Prozesse von Bedeutung ist (4.1). Die für die Kampagne innovatives-oesterreich.at relevanten Prozesse basieren zudem auf der Auswahl der Projekte (4.2), dem Programmmanagement (4.3) und Community-Building (4.4). Von Bedeutung ist, im Sinne der Realisierung von Lerneffekten für die Steuerung derartiger Kampagnen, das beobachtete organisationale Lernen, das heißt die im Verlaufe der zweiten Kampagnenphase notwendigen und vorgenommenen Zielanpassungen sowie die Bestellung eines Projektmanagers bzw. Medienkoordinators (4.5).

Die Ergebnisse der Wirkungsanalyse stehen im Zentrum des 5. Kapitels. Dabei ist zunächst von besonderem Interesse, inwieweit die Kampagne die beiden Zielgruppen „Jugendliche“ (5.1) sowie "Klein- und Mittelunternehmen“ (5.2) erreicht hat. In Form eines Exkurses wird die Wirkung der Kampagne innovatives-oesterreich.at im Vergleich zu ähnlichen Sensibilisierungskampagnen thematisiert. Vorgestellt werden im Weiteren die Ergebnisse der Wirkungsanalyse der in mehrfacher, nicht zuletzt in budgetärer Hinsicht als "Vorzeigeprojekt“ zu bezeichnenden Langen Nacht der Forschung 2005 (5.3). Abschließend werden Hinweise zur Wirkung der unter dem Dach von innovatives-oesterreich.at durchgeführten Einzelprojekte gegeben (5.4).

Eine Executive Summary mit den zentralen Befunden der begleitenden Evaluierung und den sich daraus ableitenden Handlungsempfehlungen ist dem Bericht vorangestellt. 


\section{Hintergrund und Ziele der Kampagne}

Die Kampagne innovatives-oesterreich.at ist eine der zahlreichen Awareness-Kampagnen im Bereich Wissenschaft, Forschung und Technologie, die angesichts technikkritischer Diskurse und einer als gefährdet angesehenen gesellschaftlichen Position des Wissenschaftssystem einerseits, der Karriere des Arguments „Forschung als Schlüsselfaktor im Standortwettbewerb“ andererseits, in vielen OECD-Ländern zur Verbesserung des wissenschaftspolitischen „Klimas“ lanciert werden. Auf diesen Hintergrund der Kampagne innovatives-oesterreich.at wird im Folgenden zunächst etwas ausführlicher eingegangen (2.1).

Im Weiteren wird zum besseren Verständnis der Besonderheiten der zweiten Phase der Kampagne innovatives-oesterreich.at, die Gegenstand dieser Evaluierung ist, die Genese und die Mission von innovatives-oesterreich.at dargestellt (2.2).

\subsection{Awareness-Kampagnen im Bereich Wissenschaft, Forschung und Technologie}

„Public Understanding of Science“, „Wissenschaft im Dialog“, „Engagement with Science and "Technology“, „Tage der Forschung“, „innovatives-oesterreich.at" - Awareness-Kampagnen zur Sensibilisierung einer breiteren Öffentlichkeit für das Themenfeld Wissenschaft, Forschung und Technologie sind seit einiger Zeit en vogue. ${ }^{8}$ Auch im Bereich der Wissenschaftspolitik manifestiert sich damit der allgemein zu beobachtende Trend, Implementation und Verwirklichung politischer Programme als Kampagne zu inszenieren oder zumindest auf die Unterstützung politischen Handelns durch Kampagnen zu setzen (Leggewie 2006, 110).

Awareness-Kampagnen im Bereich Wissenschaft, Forschung und Technologie reflektieren zunächst strategische Ziele der Politik und des Wissenschaftssystems, die zum einen unter dem Blickwinkel der Standortsicherung und der Position der Wissenschaft in der Gesellschaft an Legitimation, Finanzierung und Nachwuchssicherung interessiert sind, zum anderen aus demokratiepolitischen Überlegungen heraus aber auch die Notwendigkeit einer gesellschaftlichen Überprüfung und eines Dialogs über die Wissenschafts-Governance betonen. Darüber hinaus zeigen entsprechende Umfragen (vgl. Box 1) regelmäßig, dass es ein großes öffentliches Interesse gibt, mehr Informationen über Themen aus dem Bereich Wissenschaft, Forschung und Technologieentwicklung zu erhalten. Gleichzeitig wird aber auch deutlich, dass (nach wie vor) erhebliche Barrieren existieren, die den Zugang zu Wissenschaftsthemen für eine interessierte Öffentlichkeit erschweren. Aufgrund der vielen Spezialsprachen, die als Kommunikationsbarrieren zwischen Wissenschaft und Öffentlichkeit wirken, verschließen sich Entscheidungsprozesse der Wissenschaft sowie Qualität und mögliche Folgen der Forschung einer breiteren Öffentlichkeit. Angesichts eines folglich relativ hohen Maßes an Unzufriedenheit über den Informationsfluss aus der Wissenschaft scheint also das Bild des „Elfenbeinturms“ nach wie vor aktuell zu sein (Miller 2001). Zugespitzt formuliert, ist „die Distanz zwischen Wissenschaft und Öffentlichkeit heute größer denn je" - und dies, obschon das Niveau der formalen Bildungsabschlüsse gestiegen ist und der Zugang zu Informationen einfacher geworden ist und die medialen Möglichkeiten der Informationsvermittlung deutlich zugenommen haben (Felt et al. 2001, 4).

B So wies die letzte Finanzierungsrunde des Sechsten FuE-Rahmenprogramms der Europäischen Union unter dem Thema „Wissenschaft und Gesellschaft“ der Förderung des internationalen Austauschs unter den Wissenschaftskommunikator/innen und erstmals der Produktion audiovisueller Programme zur Unterstützung des Dialogs zwischen den Bürgern und der Wissenschaftsgemeinschaft in Europa 1,6 Millionen Euro zu. Zu entsprechenden Initiativen und Dialogprogrammen in Deutschland vgl. Zetsche 2004; für einen Überblick zu den Paradigmenwechseln in der Wissenschaftskommunikation vgl. Felt 2001 und Bauer et al. 2007. 


\section{Box 1: Eurobarometer „Bürger, Wissenschaft und Technologie“}

Im Jahr 2005 veröffentlichte die EU-Kommission die Studien „Die Europäer, die Wissenschaft und Technologie“ und „Die sozialen Werte, die Wissenschaft und die Technologie“. Die Ergebnisse beider Studien basieren auf Interviews, die im Rahmen der Eurobarometer-Erhebungen in jedem EU-Mitgliedsland mit einer repräsentativen Stichprobe von etwa 1.000 Personen durchgeführt wurden.

Die Ergebnisse zeigen, dass es einerseits einen verbreiteten Optimismus hinsichtlich der Beiträge der Technologieentwicklung, insbesondere der luK-Technologien und Erneuerbarer Energien, zur Verbesserung der Lebensqualität gibt, auf der anderen Seite aber auch eine erhebliche Skepsis hinsichtlich negativer Auswirkungen beispielsweise der Gentechnik.

Grafik 1 EU-Umfrage: Auswirkungen von Wissenschaft und Technologie

Wissenschaft und Techniologie werden unser Leben gesünder, einfacher und bequemer machen

Die Anwendungen der Wissenschaft und neuen Technologien werden die Arbeit interessanter machen

nur mithilfe fortgeschrittenster Technologie kann unsere Wirtschaft wettbewerbsfähiger werden

Viele Hightechprodukte sind bloß Spielereien

Die neuen Erfindungen werden immer durch die negativen Effekte der wissenschaftlichen und technologischen Entwicklung aufgewogen werden

Wissenschaft und Technologie spielen keine wichtige Rolle in der industriellen Entwicklung

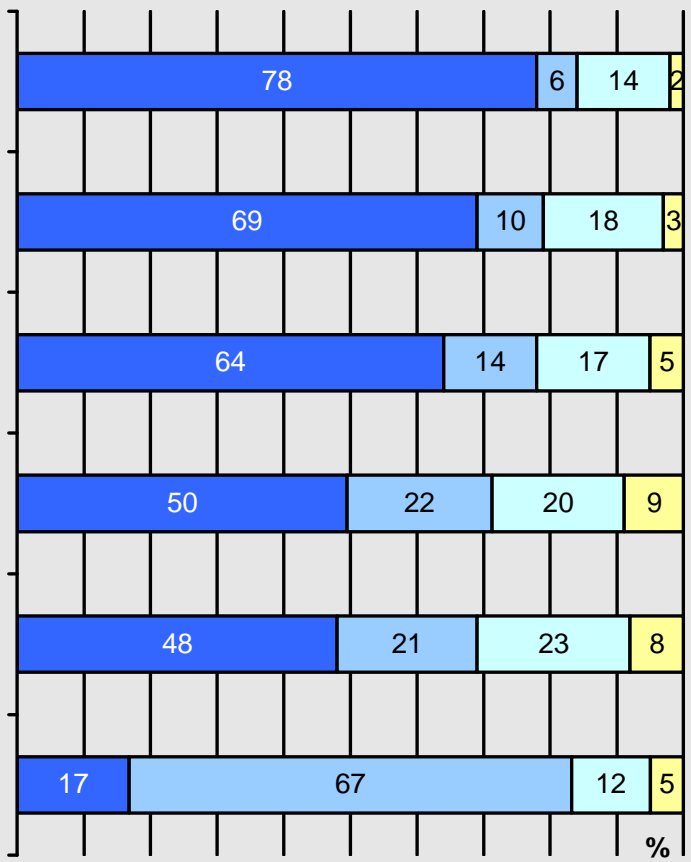

$\square$ Zustimmung $\square$ Ablehnung $\square$ Weder Noch $\square$ Keine Meinung

Ein gutes Drittel der Befragten gibt an, sie seien „sehr“ an Wissenschaft und Technologie interessiert. Gleichzeitig ist der Anteil derjenigen, die sich als "nicht interessiert“ bezeichnen, im Steigen begriffen. Dabei spielt eine Rolle, dass sich ein Großteil jener Befragten nicht in der Lage sieht, wissenschaftliche und technologische Fragen zu verstehen. Zudem gibt ein Drittel der Befragten, die Indifferenz äußern, an, das Thema bekümmere sie nicht; bemerkenswerterweise finden sich in dieser Gruppe fast 40 \% junge Menschen im Alter von 15 bis 24 Jahren.

Nur etwa jeder Zehnte der Befragten fühlt sich über neue technologische und wissenschaftliche Entdeckungen sehr gut informiert, deutlich mehr als ein Drittel aber schlecht informiert. Gleichzeitig stimmen fast $60 \%$ der Befragten der Behauptung zu, wonach „die Wissenschaftler sich zu wenig bemühen, die Öffentlichkeit über ihre Arbeit zu informieren." Wie untenstehender Grafik zu entnehmen ist, sind für mehr als die Hälfte der Befragten an den Universitäten oder in öffentlichen Labors tätige Wissenschaftler am besten qualifiziert, um die Auswirkungen von Wissenschaft und Technologien zu erklären. 


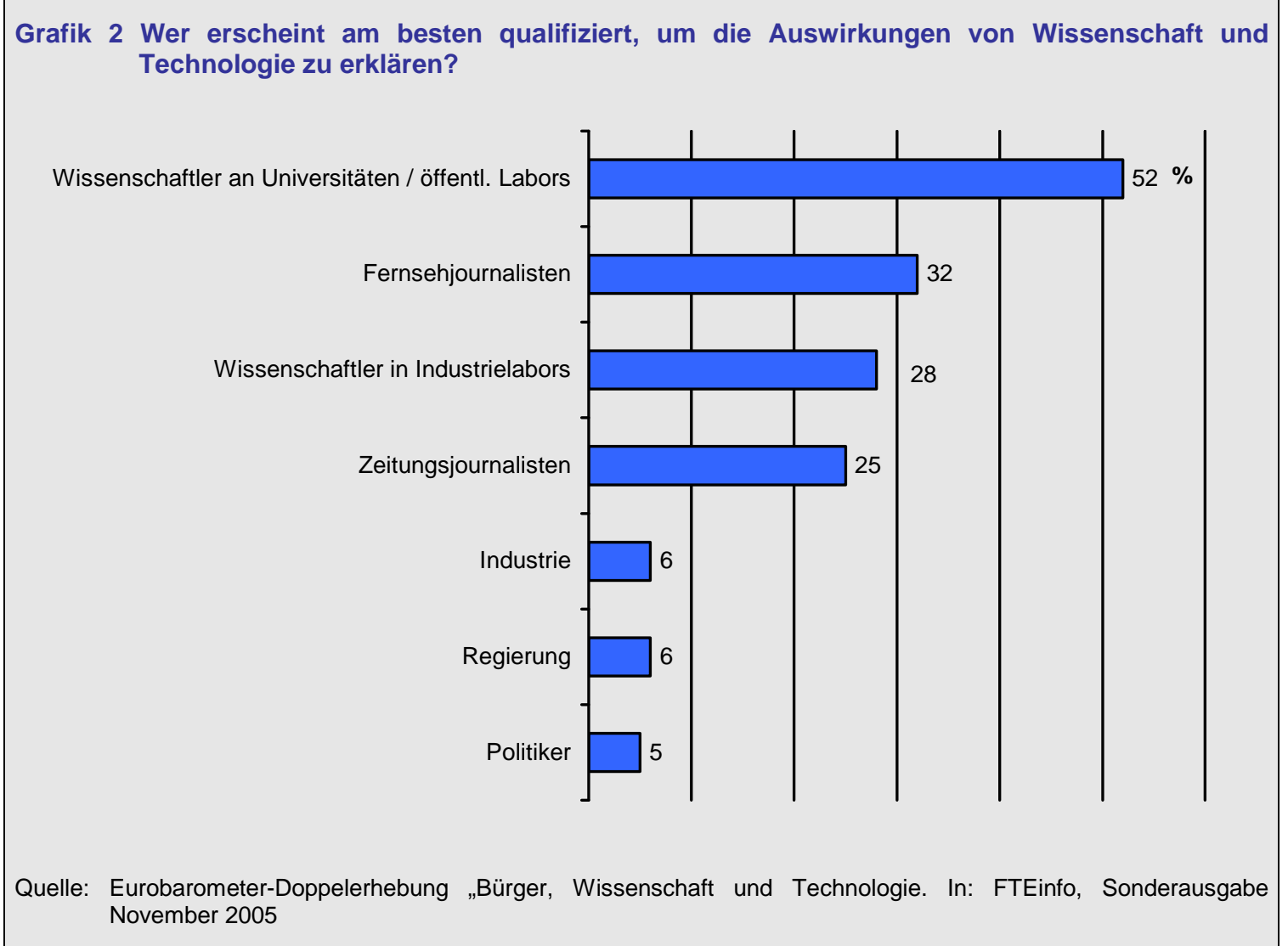

Kampagnen wie innovatives-oesterreich.at setzen hier an. Sie sollen den Stellenwert von Wissenschaft und Forschung als Schlüsselfaktor für die Sicherung von Wohlstand und $\mathrm{Be}$ schäftigung im internationalen Technologiewettbewerb verdeutlichen, über die Auseinandersetzung mit den Potenzialen, Herausforderungen, Wirkungen und Grenzen technologischer Entwicklungen den Stellenwert von Wissenschaft und Forschung und den Arbeitszusammenhang von Wissenschaft sichtbar machen und legitimieren. Im Einzelnen werden folgende Begründungszusammenhänge gesehen:

- Hervorgehoben wird, dass aufgrund zahlreicher "Schadensereignisse“ und des hohen Tempos in der Wissenschaftsproduktion zunehmend die gesellschaftliche Funktion wissenschaftlicher Forschung zur Disposition steht. Angesichts eines Vertrauensverlustes werden Nützlichkeit und Förderungswürdigkeit von Wissenschaft und Forschung in Frage gestellt Gerade im Bereich neuer Technologien wie der Genom-, Atom- oder Raumforschung stehen Befürchtungen im Raum, dass aufgrund hoher Risiken bzw. nicht absehbarer positiver „Nutzeffekte“ fehlende gesellschaftliche Akzeptanz einer breiten Nutzung und Förderung im Wege steht.

- In der Wahrnehmung wissenschaftlicher Institutionen können Misstrauen und „NichtWissen" über Bedingungen und Folgen wissenschaftlicher Arbeit zu Positions- und Reputationsverlusten in der Gesellschaft führen, so dass die Gefahr einer Beschneidung von Forschungsfreiheiten und Finanzierungsmöglichkeiten gesehen wird. Die Fähigkeit, die eigene Forschung zu vermitteln, wird daher für Forscher/innen eine zunehmend wichtige Herausforderung, von der sowohl die Attraktivität ihrer Forschungsinstitution als Ausbildungsstätte als auch die Forschungsfinanzierung abhängt.

- Aus Sicht politischer Entscheidungsträger ist die gesellschaftliche Akzeptanz von Wissenschaft und Forschung insofern von Bedeutung, da ihr einerseits für die 
Sicherung von Wohlstand und Beschäftigung im internationalen Technologiewettbewerb eine Schlüsselfunktion zukommt. Andererseits stehen Ausgaben für Wissenschaft und Forschung angesichts knapper öffentlicher Budgets unter deutlichem Legitimationsdruck; gesellschaftliche Akzeptanz ist hier Voraussetzung für eine dauerhafte Finanzierung. Zudem wird die Sorge um die gesellschaftliche Position der Wissenschaft auch dadurch gespeist, dass politische Entscheidungen über wissenschaftliche Expertise legitimiert werden.

- Aus Sicht von Unternehmen steht im Vordergrund, dass es aufgrund verbreitet "technikaverser" Berufswahlentscheidungen von Schulabsolventen gerade im naturund ingenieurwissenschaftlichen Bereich an Nachwuchs mangelt. Argumentiert wird, dass dieser Nachwuchsmangel durch die Abwanderung aussichtsreicher jüngerer Forscher und Wissenschaftler noch akzentuiert wird. Folglich steht die Befürchtung im Raum, dass es in den Unternehmen zu einer Einschränkung der Umsetzung wissenschaftlicher Erkenntnisse in neue Produkte kommt, wodurch auf mittlere Sicht die Innovationsfähigkeit und damit die Wettbewerbsposition der Unternehmen erodieren könnte.

Awareness-Kampagnen, so die Erwartung, sind geeignet, über ein gesteigertes Interesse und intensivierte Auseinandersetzung einer breiteren Öffentlichkeit mit Wissenschaftsthemen vertrauensbildend zu wirken und so zu einem günstigeren wissenschaftspolitischem Klima beizutragen. Erfolg bzw. Wirksamkeit solcher Kampagnen hängen davon ab, dass die Einzelmaßnahmen Bestandteil eines Gesamtkonzeptes sind, sie über einen längeren Zeitraum wirken, die Botschaften zielgruppengerecht zugeschnitten und positiv besetzt sind und solche Medien eingesetzt werden, die die Zielgruppen auch erreichen.

\section{Box 2: PR-Kampagnen und Wissenschaftskommunikation}

„Kampagnen sind dramaturgisch angelegte, thematisch begrenzte, zeitlich befristete kommunikative Strategien zur Erzeugung öffentlicher Aufmerksamkeit, die auf ein Set unterschiedlicher kommunikativer Instrumente und Techniken - werbliche Mittel, marketing-spezifische Instrumente und klassische PR-Maßnahmen - zurückgreifen. Ziele von Kampagnen sind: Aufmerksamkeit erzeugen, Vertrauen in die eigene Glaubwürdigkeit schaffen und Zustimmung zu den eigenen Intentionen und/oder Anschlusshandeln erzeugen." (Ulrike Röttger, Kampagnen. In: O. Jarren, U. Sarcinelli, U. Saxer (Hg.): Politische Kommunikation in der demokratischen Gesellschaft. Ein Handbuch mit Lexikonteil. Opladen, Wiesbaden 1998, S.667)

"Science communication is defined as the use of appropriate skills, media, activities, and dialogue to produce one or more of the following personal responses to science: Awareness, Enjoyment, Interest, Opinion-forming, and Understanding." (T.W. Burns, D.J. O'Connor, S.M. Stocklmayer, Science Communication: a contemporary definition. In: Public Understanding of Science, Vol.12, 2003, 183-202)

Die Konzeption solcher Kampagnen kann auf Befunde der Fachdiskussion um Wissenschaftskommunikation rekurrieren. Betont wird hier insbesondere, dass

„das Bild, das sich die Wissenschaft in ihren vielfältigen Bemühungen um öffentliche Aufmerksamkeit von ihrer Öffentlichkeit macht, offenbar ... noch weitgehend der spezifisch akademischen Sicht [entspringt, d.V.], die in den 1930er bis 1960er Jahren geprägt worden ist. Die groß angelegten, auf die massenmediale Aufmerksamkeit für besondere Events ausgerichteten Werbekampagnen gehen noch immer von einer unspezifischen und unstrukturierten Öffentlichkeit aus, die doch in Wirklichkeit weder homogen noch passiv ist. Erst ganz allmählich wird diese Öffentlichkeit zumindest rhetorisch "engagiert", in einen "Dialog" eingebunden." (Weingart 2004, 19) 
Jenseits der Bedeutung der Heterogenität der Adressaten solcher Kampagnen wird argumentiert, dass die Vermittlung von wissenschaftlichem Wissen in die Öffentlichkeit kein einseitiger Prozess ist, in dem im Sinne einer „Aufklärung von oben“ vorrangig das „Übersetzungsproblem" sowie das Interesse und die Aufnahmefähigkeit „der“ Öffentlichkeit zu thematisieren sind (Felt 2000). Vielmehr kann argumentiert werden, dass Wissenschaft als von Interessen durchdrungenes System einerseits Auswirkungen auf nicht der Wissenschaft angehörende Akteure, deren Werte, Identitäten und Interessen hat, andererseits aber auch selbst von gesellschaftlichen Veränderungen beeinflusst wird:

\begin{abstract}
„People experience these in the form of material social relationships, interactions and interests, and thus they logically define and judge the risk, the risk information, or the scientific knowledge as part and parcel of that social package." (Wynne 1992, 282).
\end{abstract}

Thematisiert wird damit die Einbeziehung der Wissenschaft in öffentliche Diskurse, in denen etwa wissenschaftspolitische Prioritätensetzungen und Verwertungsentscheidungen demokratisch legitimierten Aushandlungsprozeduren unterliegen.

Allerdings sehen sich auf einer grundsätzlichen Ebene auch zielgruppenorientierte, auf Interaktion und Partizipation der Adressaten setzende Awareness-Kampagnen ähnlicher Kritik ausgesetzt wie eher traditionell angelegte Werbekampagnen:

- Es scheint einiges dafür zu sprechen, dass die zugrunde gelegte Annahme, ein über die Kampagne bewirkter höherer Kenntnisstand über den Gegenstand erzeuge bei den Adressaten eine höhere Zustimmung, ein akademisches Vorurteil darstellt. Vielmehr zeigt das Beispiel Gentechnik, dass, je weniger ausgeprägt Wissensstand der Befragten ist, desto größer ist "die Wahrscheinlichkeit, dass die Befragten meinungslos sind." (Hampel, Renn 1999, 387).

- Kritisiert wird zum anderen, dass sich Awareness-Kampagnen im Bereich wissenschaftlicher Forschung primär an die ohnehin sensibilisierte, aufmerksame Öffentlichkeit (attentive public) richten, also an jene etwa $10 \%$ der Befragten mit einer generell positiven Einstellung zu Wissenschaft und Technik; zudem werde diese Gruppe nur dann in die wissenschaftspolitischen Entscheidungen einbezogen, wenn auf der Ebene der Entscheidungsträger wissenschaftspolitische Entscheidungen nicht konsensfähig sind (Weingart 2004).

- Problematisiert wird, dass solche Kampagnen der Medialisierung der Wissenschaft Vorschub leisten. Da die zeitliche Ereignisstruktur auf die Regeln und Routinen des Mediensystems ausgerichtet ist und auf eine möglichst hohe Medienresonanz zielt, gerät die Wissenschaft zunehmend in die Abhängigkeit von den Vermittlungslogiken der Medien, beispielsweise hinsichtlich der Aktualitätstempi oder der Themenkonjunkturen - mit der Gefahr, dass Neuigkeit zur hohlen Kardinaltugend wird (Weingart 2004).

\title{
2.2 Die Kampagne innovatives-oesterreich.at und ihre Mission: Von der Ratsempfehlung zur Neuorientierung in der 2. Kampagnenphase
}

Die Kampagne innovatives-oesterreich.at geht wesentlich auf die Initiative des Rates für Forschungs- und Technologieentwicklung (Rat für FTE) zurück. Seit seiner Konstituierung im Jahr 2000 zählt der Rat für FTE die Förderung eines öffentlichen Klimas, das die Entwicklung von Wissenschaft, Forschung und Technologie begünstigt, zu seinen Prioritäten:

„Wir betrachten das Humankapital als die wichtigste Voraussetzug für die Wettbe-
werbsfähigkeit der österreichischen Wirtschaft und Wissenschaft auf Basis der
Freiheit von Forschung und Lehre. Das Vorhandensein von qualifizierten und moti-
vierten Mitarbeitern ist von zentraler Bedeutung. Voraussetzung dafür ist es, bei 


\section{den Staatsbürgern das Verständnis für Wissenschaft, Forschung und Technologie zu wecken und zu fördern." 9}

In seiner Empfehlung vom 20. November $2001^{10}$ an die österreichischen Bundesregierung nennt er die Einführung eines Bewusstseinsbildungsprogramms zum Thema „Forschung - Entwicklung - Innovation“ für die Jahre 2001-2003, gekoppelt mit der Einführung eines neuen Organisationsmodells, das in einem Lenkungsausschuss alle am Programm strategisch beteiligten Institutionen (Ressorts, Rat, Interessensvertretungen, Medien) zusammenführen soll. Damit ist der Grundstein für die erste Kampagne gelegt. Eine Verlängerung der Kampagne wird 2003 vom Rat empfohlen, diese zweite Kampagne ist Gegenstand der vorliegenden Evaluierung.

\section{Box 3: Auszug aus der Empfehlung des Rats für Forschung und Technologie- entwicklung vom 25. November 2003, Zur Förderung von Public Awareness für Forschung, Technologie und Innovation}

Der Rat für FTE hat am 11. August 2003 empfohlen, $2 \%$ des Volumens des Offensivprogramms II im Strategiefeld „Dialog zwischen Wissenschaft / Forschung / Technologieentwicklung / Innovation und Gesellschaft“ zum Einsatz zu bringen.

„Der Rat für FTE empfiehlt eine Fortsetzung des Programms www.innovativesoesterreich.at. (...) Dieser Mittelpool ist aus dem mit den drei Ressorts vereinbarten Finanzierungsrahmen zu bilden und für die Bündelung von Einzelmaßnahmen einzusetzen. Die Mittelverteilung ist von vornherein auf Zielbereiche aufzugliedern (Public Relations, Werbung, Veranstaltungen, Medienkooperationen, Begleitforschung, EURelevanz, etc.). (...) Es ist ein Programmkomitee einzusetzen, das aus dem Rat für FTE sowie je einem/r Vertreter/in der drei Budenministerien besteht."

Die Ratsempfehlung hat jedoch Awareness-Aktivitäten nicht erst geschaffen, auf Bundesebene hatten die drei in Forschung und Innovation involvierten Ressorts schon vor dem Start der Kampagne unterschiedliche Projekte beziehungsweise Ausschreibungen lanciert. Diese Aktivitäten sollten fortan unter der Dachmarke innovatives-oesterreich.at als so genannte „Ressortprojekte“ geführt werden. Im Gegenzug zu den erhöhten Koordinationskosten und den aus der Koordination folgenden Verbindlichkeiten wurde längerfristig durch die $2 \%$ Klausel (vgl. Box 3) ein Budget garantiert, das in der Größenordnung die bis dahin aufgelegten Awareness-Budgets übertraf und eine längerfristige Planung ermöglichte.

Auf dem Wege der Umsetzung zeigte sich allerdings, dass die Aktivitäten eine gewisse Eigendynamik entwickelten und es insbesondere zu einer nur schwer einzufangenden Dynamik zwischen Ressortinitiativen und ressortübergreifender Kampagne kam: Neben laufenden Projekten und Programmen der Ressorts existierten Projekte aus der ersten Kampagnephase, die in die zweite Kampagnephase übernommen werden sollten, da sie längerfristig angelegt sind; zudem gab es insbesondere ein großes Projekt, das unter dem Titel „Fest der Wissenschaft und Technologie" im Mai 2003 vom BMVIT ausgeschrieben und im September 2003 vergeben wurde, um dann verzögert und unter dem neuen Titel „Lange Nacht der Forschung“ im Herbst 2005 stattzufinden.

Die erste Kampagne (2001-2003, Budget 7,5 Mio. €) firmierte unter dem Slogan „Auf die Birne kommt es an". Sie kombinierte einen Ideenwettbewerb und Ressortprojekte, förderte die Entwicklung eines Logos und finanzierte insbesondere eine Werbekampagne. Die Evaluierungsergebnisse waren teilweise ernüchternd, da die Werbung zwar gesehen, aber oft nicht verstanden wurde, und nicht ausreichend nachzuvollziehen war, dass sie tatsächlich das öffentliche Bewusstsein für Forschung- und Innovationstätigkeit geschärft, beziehungsweise

\footnotetext{
9 Rat für FTE: Vision 2005. Hervorhebung durch die Autoren.

10 Alle Empfehlungen des Rates für FTE sind auf der Homepage des Rates unter der Rubrik Dokumente zu finden.
} 
positiv gestimmt hätte.". Die mangelnde Zielerreichung konnte nicht zuletzt, da „klassische“ Werbung über Fernsehspots und Plakate kostspielig ist, auf budgetäre Restriktionen zurückgeführt werden.

Vor dem Hintergrund dieser Erfahrungen einigte man sich für die zweite Kampagnenphase (2004-2006, Budget 12 Mio. €) auf eine Neuorientierung: An Stelle großflächiger klassischer Werbemaßnahmen wurde nun verstärkt auf zielgruppenorientierte Aktionen, PR und Medienarbeit gesetzt; zudem wurde ein neues Logo - als „Dachmarke“ - eingeführt. Um die Transparenz bei der Vergabe zu erhöhen, erfolgte eine öffentliche Ausschreibung der Kampagne. Als Zielgruppen wurden Jugendliche sowie kleine und mittlere Unternehmen (KMU) definiert: Die Begründung für diese speziellen Zielgruppen bezog sich zum einen auf den Befund, dass sich junge Menschen in Österreich in viel zu geringem Maße für die Ausbildung im natur- und ingenieurwissenschaftlichen Bereich interessieren. Zum anderen konnte argumentiert werden, dass Klein- und Mittelunternehmen nicht nur $95 \%$ der österreichischen Unternehmen ausmachen, sondern dass hier auch noch ein erhebliches Innovationspotenzial "schlummert“, das es zu aktivieren gilt.

Jenseits der Orientierung auf diese beiden Zielgruppen definiert sich die Mission der zweiten Phase der Kampagne innovatives-oesterreich.at über folgenden Zielkatalog ${ }^{12}$ :

- Wissen und Innovation für die Öffentlichkeit zugänglicher machen;

- gezielt öffentliches Interesse für ein breites Spektrum von verschiedenen Wissenschaften und Technologien sowie Forschende - insbesondere Frauen - ermöglichen;

- Interesse und Neugierde für Forschung und Innovation wecken, insbesondere bei jungen Menschen;

- Hemmschwellen und Berührungsängste gegenüber Wissenschaft, Forschung und Technik abbauen;

- die Übersetzung von fachsprachlich formulierten Aussagen/Erkenntnissen in die Alltagssprache fördern;

- Methoden und Vorgangsweisen von Forschung und Innovation zu demonstrieren, und zur Diskussion stellen;

- die Kommunikationskompetenz von FTI-Institutionen hin zur Öffentlichkeit verbessern;

- qualitätsvollen Wissenschaftsjournalismus fördern;

- direkte Begegnungen und Erlebniswelten bereitstellen;

- die internationale Vernetzung verstärken.

11 In der Zusammenfassung der Ergebnisse der empirischen Studie, die von Prof. Dr. Günter Schweiger et al. im Februar 2003 fertig gestellt wurde, kann man lesen, dass die Stärken der Kampagne die folgenden waren: „Die Werbung ist aufgefallen./ Ein Viertel der Befragten erinnert sich an die Wortbildmarke./ Der TV-Spot und die Anzeige werden als humorvoll und sympathisch beurteilt". Demgegenüber sind die folgenden Schwächen genannt: „Der Spot steigert das Interesse an Forschung und Entwicklung kaum./ Der Spot lädt nur wenig zum Besuch der Homepage ein." Insbesondere mit der ersten Schwäche ist das Ziel der Kampagne im wesentlichen verfehlt, was die genannten Autoren zu der Schlussfolgerung führt, „Anzeigen und Homepage zielgruppenadäquat zu adaptieren, um das Interesse an Forschung und Entwicklung zu wecken."

12 In den Ausschreibungsunterlagen (2004) gelisteter Zielkatalog. 



\section{Strukturen der Kampagne innovatives-oesterreich.at}

Das organisatorische Setting bildet ein strukturprägendes Merkmal der Kampagne innovativesoesterreich.at. Von Bedeutung ist hier zunächst das neue Modell der Koordination (3.1), das auch das Handeln der beteiligten Akteure wesentlich beeinflusst (3.2) und die Strukturen der im Rahmen der Kampagne durchgeführten Projekte geprägt hat (3.3).

\subsection{Ein neues Modell der Koordination}

Im Februar 2004 wurde ein Programmkomitee für die Kampagne innovatives-oesterreich.at eingerichtet, bestehend aus BMBWK, BMVIT, BMWA und Rat für FTE. Das Programmkomitee wird vom Rat koordiniert und entsprechend der Geschäftsordnung geführt. Im August 2004 wurde die Mittelaufteilung für die Kampagne innovatives-oesterreich.at fixiert, diese folgt der in Grafik 3 dargestellten Struktur. Von den 12 Mio. $€$, die für die zweite Kampagnenphase vorgesehen waren, wurden 6,5 Mio $€$ als ressortübergreifendes Budget und 5,5 Mio. $€$ für Ressortprojekte veranschlagt. Aus dem ressortübergreifenden Budget wurden 3 Mio. $€$ für die Ausschreibung der Kampagne in 4 Modulen und 2,6 Mio. $€$ für das Fest der Forschung (späterhin X.PERIMENTA ${ }^{13}$ ) gebunden.

Grafik 3 Struktur der Förderungen im Rahmen von innovatives-oesterreich.at

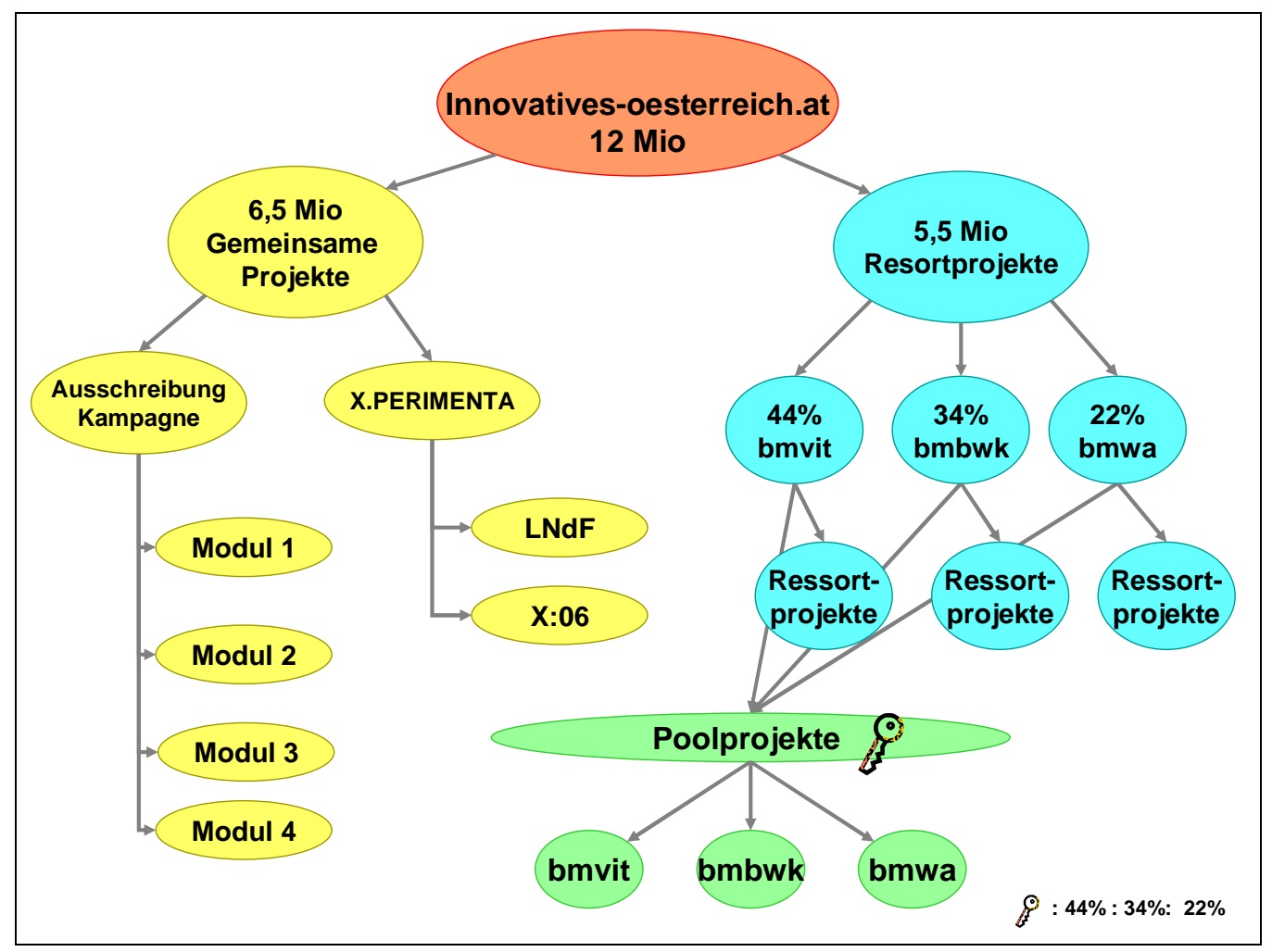

Die Organisation der Plattform innovatives-oesterreich.at ist in der österreichischen forschungspolitischen Landschaft insofern etwas Neues, als die interministerielle Koordination über einen reinen Informationsaustausch hinausgeht: Der Lenkungsausschuss hat Entscheidungsbefugnis bei Fragestellungen, bei denen gemäß traditioneller Koordination eine Bewilligung der jeweili-

13 Die Benennung dieses Ereignisses hat sich über die Zeit gewandelt. Das „Fest der Wissenschaft und Technologie“ wurde zur "Langen Nacht der Forschung“. 
gen ministeriellen Hierarchie eingeholt werden müsste. So können größere Projekte unmittelbar durch den Lenkungsausschuss beschlossen werden. ${ }^{14}$

Es stellte sich jedoch heraus, dass die Koordination zu schwach bzw. unvollständig konzipiert war. Aufgaben landeten bei der Geschäftsstelle des Rats für FTE, für die diese nicht vorgesehen war und auch nur über unzureichende personelle Ressourcen verfügte. Dementsprechend zeigten sich mehrere Akteure des Lenkungsausschusses unzufrieden mit der Koordination der Kampagne. Bemängelt wurden hier beispielsweise zu häufig stattfindende Sitzungen oder der Umstand, dass Entscheidungen der Plattform nicht ausreichend formell getroffen und insbesondere nicht immer eingehalten wurden. Die unzureichende Koordination spiegelt sich auch in den Einschätzungen der Projektträger/innen wider. Wie Grafik 4 verdeutlicht, gab es aus Sicht der Projektträger keine eindeutige Zuordnung der Koordinationsfunktion zu einem bestimmten Akteur: Die Aufgaben „Koordination und Programmmanagement“ werden quasi zu gleichen Teilen dem Rat für FTE, dem Lenkungsausschuss und der LeadAgentur Trimedia zugeordnet, es gibt also keine einheitliche Wahrnehmung der Verantwortlichkeiten.

Grafik 4 Zuordnung der Kampagnenfunktion „Koordination, Programmmanagement“ zu Kampagnenakteuren

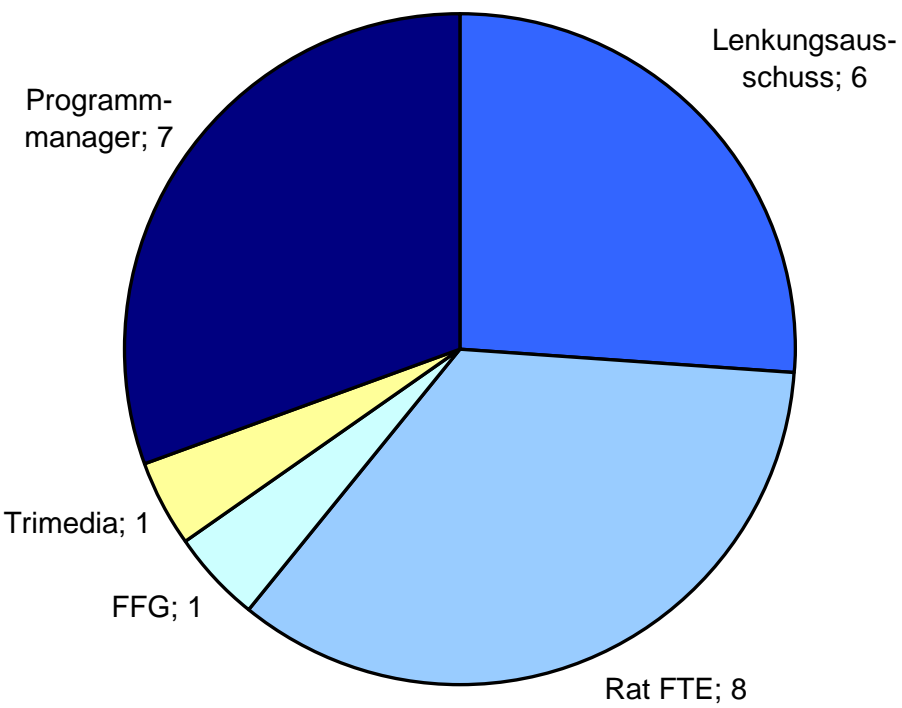

Anmerkungen:

$\mathrm{N}=23$

Anzahlen der Antworten auf die Frage: „Welcher Akteur sollte die Funktion „Koordination/Programmmanagement“ Ihrer Meinung nach ausüben?"

Quelle: KMU FORSCHUNG AUSTRIA, Online-Befragung (Projektträger); Mai/Juni 2006

Diese Wahrnehmung hängt vermutlich damit zusammen, dass die Zuständigkeiten tatsächlich auf unterschiedliche Akteure verteilt, gleichzeitig jedoch unzureichend vertraglich festgelegt waren, so dass es sowohl zu Doppelgleisigkeiten als auch zu Lücken im System kam. Beispielsweise hatten beide Lead-Agenturen Kontakte mit anderen Projektträgern, beide sammelten Informationen über Projekte ein, jedoch war es so dem Evaluationsteam auf Ebene des Programmmanagements nicht möglich, eine vollständige Zusammenstellung mit einem detaillierten Ablauf- und Zeitplan aller Projekte zu erhalten. Die Berichtlegung über die Projekte

14 Seit seiner Gründung hat der Rat für FTE an verschiedener Stelle zu mehr Koordination zwischen den für FuE zuständigen Ressorts aufgerufen. Die Befugnisse des Programmkomitees sind jedoch einmalig, da das Komitee für „die Generierung eines zielgerichteten Maßnahmenkatalogs zur Umsetzung der Empfehlung des Rates für Forschung und Technologieentwicklung (RFT) vom 25. November 2003“ zuständig ist. Die Zuständigkeit der einzelnen Ressorts wurde in dieser Sache also dem Gremium, in dem diese Ressorts vertreten sind, zugewiesen. 
war einerseits für die Kampagnenprojekte verpflichtend an die FFG, jedoch waren es die LeadAgenturen, die Projektberichte für ihre Arbeit benötigten, und zwar nach einem anderen Format als es die FFG forderte. Auch die Koordination zwischen Kampagnenprojekten und Ressortprojekten war teilweise lückenhaft, so wurde beispielsweise die AWS, die Ressortprojekte des BMWA seit vielen Jahren betreut, nur wenig in die Gesamtkampagne eingebunden.

Grafik 5 illustriert das Auseinanderfallen von Funktionen anhand der Aufgaben „Beauftragung“ und „Koordination“. Sie stellt schematisch die Organigramme für diese beiden Bereiche des Programmmanagements dar. Die Pfeile weisen auf die übergeordnete Zuständigkeit von Akteuren hin. Bezüglich des Lenkungsausschusses ist darauf zu achten, dass manche Zuständigkeiten inm selbst, andere seinen Mitgliedern zugeordnet sind. Akteure auf gleichem Niveau sind in ihren Zuständigkeiten gleichgestellt: es ist also insbesondere auffällig, dass die LeadAgenturen zwischen den beiden Darstellungen "das Niveau wechseln“, dass die AWS in der Koordination aus dem Bild fällt, während ein Medienkoordinator hinzukommt.

Auf Basis der Analyse von Auftragsunterlagen einerseits und der Auswertung der mit involvierten Akteuren geführten Interviews ergibt sich die Einschätzung, dass folgende Aspekte als problematisch anzusehen sind:

- Der Rat für FTE hat ausschließlich die Rolle des Koordinators, ist jedoch nicht Auftraggeber.

- Die FFG ist Auftraggeberin, ohne eine Koordinationsrolle innezuhaben.

- Die Lead-Agenturen sind als beauftragte Projekte den anderen Kampagnenprojekten gleichgestellt, spielen jedoch in der Koordination eine übergeordnete Rolle. Bezüglich der Ressortprojekte haben sie eine schlechtere „Zugriffsmöglichkeit“ in der Koordination als bei Kampagnenprojekten.

- Der Medienkoordinator bzw. „Koordinator für begleitende Maßnahmen“ wurde erst zu einem späteren Zeitpunkt der zweiten Kampagnenphase beauftragt.

- Die Ressorts behalten eine Koordinationsfunktion bei den Ressortprojekten. Diese werden im Rahmen des Lenkungsausschusses nur durch gegenseitige Information abgestimmt (um unerwünschte Überlappungen zu vermeiden), weiters sollen sie unter dem Label (und mit dem Logo) innovatives-oesterreich.at laufen, und können auf gemeinsame PR, Medienarbeit und Internetpräsenz zurückgreifen. Das Ausmaß des Auftrags an die LeadAgenturen, diese Projekte einzubinden, ist nicht eindeutig, es bleiben somit unterschiedliche Einschätzungen über die Rolle der Dachmarke nebeneinander bestehen.

Probleme, die zumindest teilweise auf die komplexe Struktur und fehlende Transparenz bei der Zuordnung von Verantwortlichkeiten zurückzuführen sind, ergaben sich insbesondere beim Informationsfluss, der Berichtlegung, dem Projektmanagement und dem Timing der Medienarbeit. 

tionsfunktion

\section{Beauftragung}

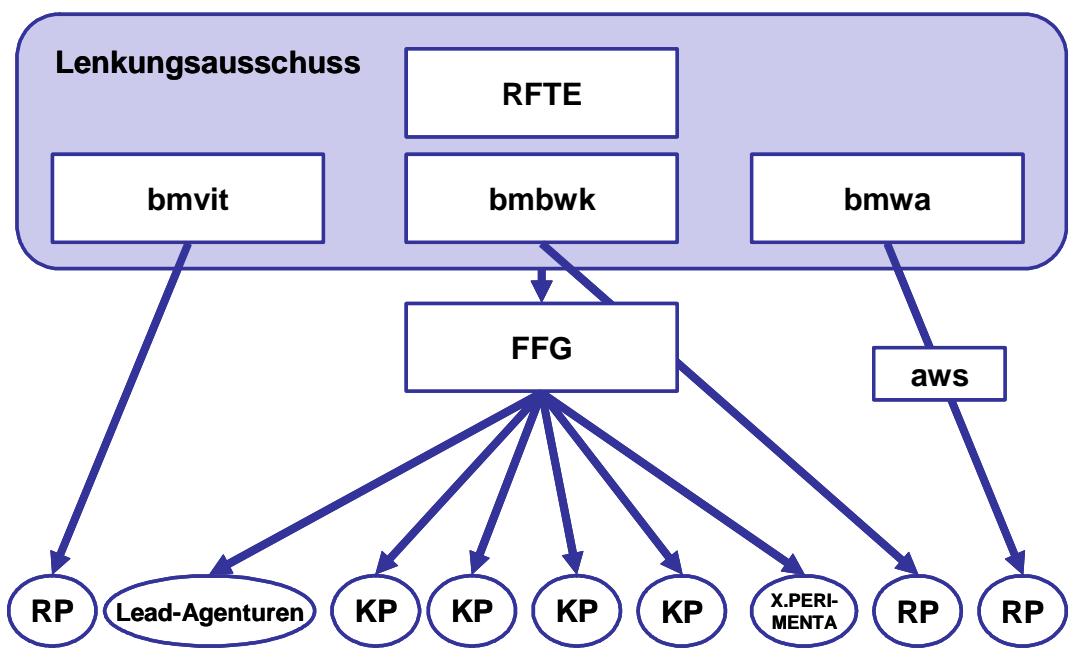

\section{Koordination}

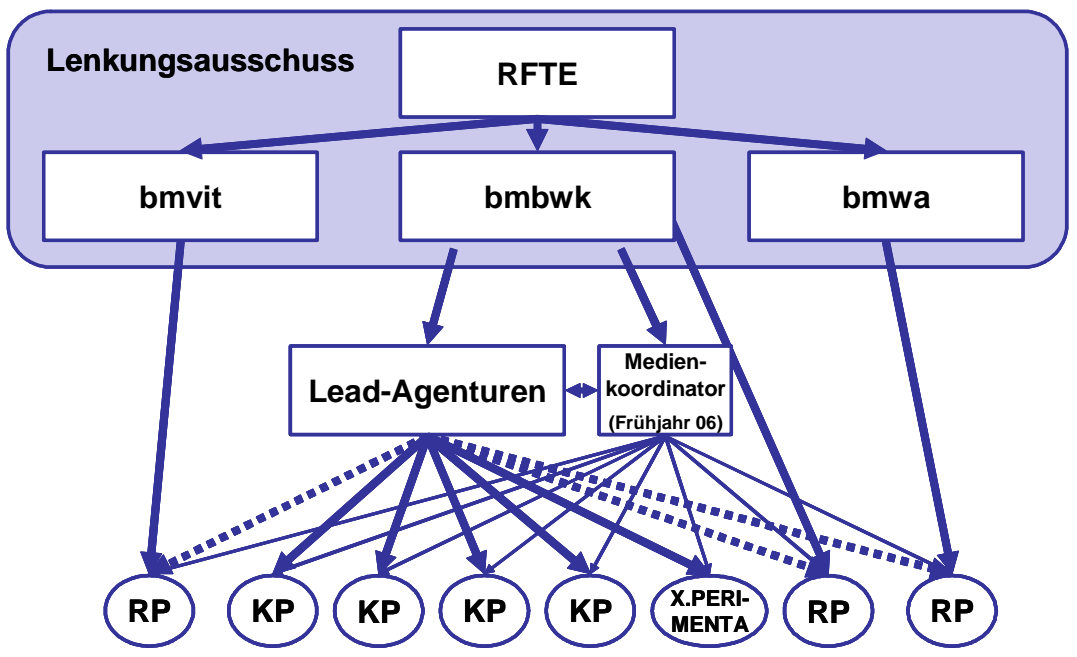

Anmerkung:

RP: Ressortprojekt; KP: Kampagnenprojekt

Es zeigt sich allerdings, dass es einen Bedarf an Programmmanagement auf folgenden Ebenen gibt:

- Im Vorlauf des Programms, bei der Definition strategischer Ziele, wird ein Mediator zum Interessensausgleich benötigt. Der Rat für FTE hat diese Funktion insbesondere bei der Vorbereitung der Ausschreibung eingenommen. Es erwies sich aber auch während der Laufzeit der Kampagne als notwendig, zu vermitteln. Brisanterweise hatte der Rat für FTE hier eine Doppelfunktion, da er einerseits durch seine Mission der strategischen Beratung der Bundesregierung eine Meinung zu der Kampagne und ihrem Verlauf ausdrückt, als auch mit einem Vertreter der Geschäftsstelle als Vorsitzendem des Lenkungsausschusses die Rolle des Mediators innehat. Im Winter 2005/2006 führte dieses Spannungsverhältnis zu einer Krise, in der der Rat es offensichtlich gerne gesehen hätte, wenn die Kampagnenmittel im Sinne seiner eigenen Einschätzung von „Sichtbarkeit“ eingesetzt würden - und dies zu einem Zeitpunkt, als die Mittel eigentlich schon auf Basis gemeinsam getragener 
Entscheidungen vergeben waren. Sowohl im Rahmen von Interviews als auch bei öffentlichen Veranstaltungen getätigten Aussagen von Vertreter/innen des Rates kann entnommen werden, dass ein Selbstverständnis existiert, nachdem der Rat für FTE „Mittel vergibt“ bzw. Projekte finanziert - eine Einschätzung, die keine zutreffende Rollendefinition widerspiegeln dürfte. Für weitere Kampagnen erscheint es daher wichtig, die Rolle des Rates als beratendes Organ im Vorlauf, und als Mediator im Zuge eines ressortübergreifenden Programms klar abzugrenzen.

- Theoretisch sollte ein Projektmanager laufend für die Erstellung und Einhaltung eines Zeitplans zuständig sein, Meilensteine definieren, Finanzströme kontrollieren und die Berichtlegung koordinieren und sicherstellen. In der Realität der zweiten Kampagnenphase waren diese Aufgaben jedoch unzureichend definiert, sodass keiner der Akteure sich a priori dafür zuständig fühlte, und mehrere Akteure, insbesondere jedoch die Geschäftsstelle des Rat für FTE, als Projektmanager in Anspruch genommen wurde. Den LeadAgenturen werden einige mit dem Projektmanagement verbundenen Aufgaben zugeschrieben, teilweise werden sie von ihnen auch wahrgenommen, jedoch sehen sich die Lead-Agenturen durch ihre mangelnden Zugriffsmöglichkeiten auf die Projekte nicht in der Lage, ein vollständiges Projektmanagement zu übernehmen, auch war das nicht Teil ihrer Anbote.

- Um die Dachmarke innovatives-oesterreich.at zu kommunizieren und so einen Multiplikatoreffekt zu erreichen, kommt der Koordination der Medienarbeit eine Schlüsselrolle zu. Hier gibt es insgesamt drei Zuständigkeiten: die Agenturen, die Pressestellen der jeweiligen Ressorts (insbesondere für Ressortprojekte), und der im Frühjahr 2006 beauftragte Koordinator.

- Der Informationsaustausch läuft typischerweise auf vielen Gleisen, insbesondere in einer Community, bei der sich der Großteil der Projektträger/innen schon vor der Kampagne kannte ${ }^{15}$. Im Sinne der Koordination hat der Informationsaustausch die Aufgabe, erstens alle relevanten Informationen zu sammeln, sie zweitens an alle involvierten Beteiligten weiterzugeben und drittens Informationen an ein breiteres Publikum weiterzuleiten. So organisierte eine Lead-Agentur ein Kick-off Treffen aller Projektträger/innen zur Präsentation der Kampagne und der einzelnen Projekte, und koordinierte regelmäßige Meet-up's. Hinsichtlich der elektronischen Informationsaufarbeitung kam es zu Doppelgleisigkeiten, da zwei Agenturen mit der Bereitstellung einer Internetplattform beauftragt wurden, jedoch in keinem der Verträge die Koordination mit der jeweils anderen Agentur und Plattform festgeschrieben war.

Im vorliegenden Kontext ist folgender Sachverhalt aufschlussreich. Fragt man die Projektträger/innen, welche Kampagnenfunktion zu welchem Kampagnenakteur zuzuordnen sind und welche Bedeutung sie den Funktionen für den Kampagnenerfolg beimessen, ergibt sich ein recht heterogener Befund: Grafik 6 verdeutlicht, dass eine große Mehrheit der Projektträger/innen Koordination, Programmmanagement, Community-Building, ein Werbekonzept, insbesondere aber ein gemeinsames Logo und PR-Arbeit für wichtig bis sehr wichtig halten. Die Zuordnung zu einem verantwortlichen Akteur fällt jedoch nur bei den Funktionen Werbekonzept und PR-Arbeit - mit der Zuweisung dieser Aufgabe an die Lead-Agentur Trimedia - relativ eindeutig aus. Im Gegensatz dazu sind Informationsaustausch und Community-Building in der Wahrnehmung der Projektträger/innen alles andere als zentralisiert. Die Verfügung über ein gemeinsames Logo wird von mehr als der Hälfte der Befragten als sehr wichtig eingeschätzt, die Meinungen, wer dafür zuständig ist, gehen aber auseinander. Es ist insbesondere auch auffällig, dass dem „Programmmanager“, den es zum Zeitpunkt der Befragung gar nicht gab,

15 Fast drei von vier Projektleitern hatten bereits vor ihren Projektaktivitäten im Rahmen der Kampagne Kontakte zu anderen involvierten Projektleitern bzw. kennt diese aufgrund des Kick-off Meetings zu Beginn der Kampagne. An die zwei Drittel sind über die Meet-up's im Rahmen der Kampagne von anderen Projekten informiert und nur $15 \%$ über die Plattform technology log. Aufgrund von Projektaktivitäten im Rahmen der Kampagne andere Projekte zu kennen geben ein Drittel der Befragten an. Vgl. auch Grafik 18. 
eine nicht zu vernachlässigende Rolle in diesem wie auch in anderen Punkten zugeschrieben wurde.

Grafik 6 Zuordnung von Kampagnenfunktionen zu Kampagnenakteuren und Bedeutung der Kampagnenfunktionen - die Sicht der Projektträger/innen

\section{Zuordnung}

口RFTE DLA DFFG DTrimedia Duma Dtechnologylog DProgrammmanager

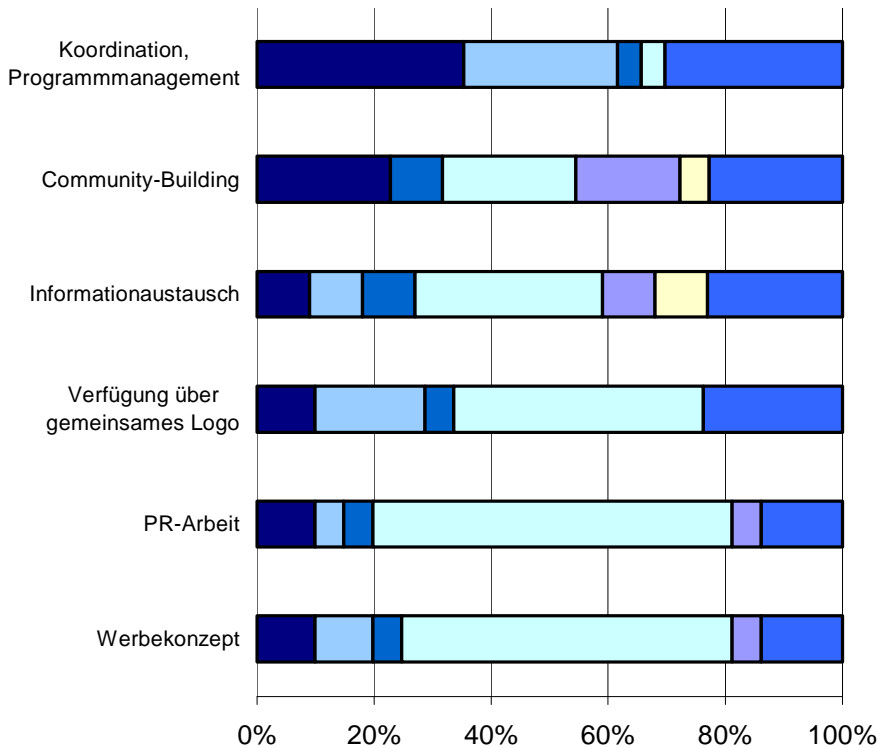

Bedeutung

$\square$ sehr wichtig $\square$ wichtig $\square$ weniger wichtig

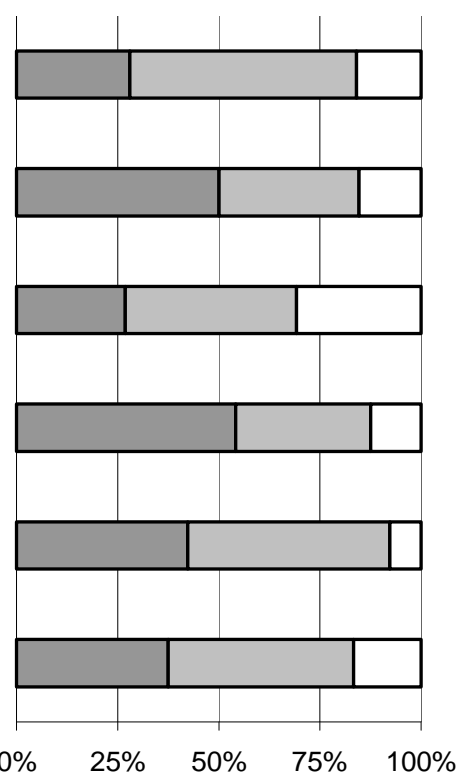

Anmerkungen:

$\mathrm{N}=21-23$ bzw. $24-26$

Anteile der Antworten auf die Frage „Welche Funktion hat aus Ihrer Sicht die Dachkampagne innovatives-oesterreich.at. und welche Bedeutung hat diese Funktion?" bzw. „Welche der folgenden Akteure sollten diese Funktionen Ihrer Meinung nach ausüben?".

Quelle: KMU FORSCHUNG AUSTRIA, Online-Befragung (Projektträger); Mai/Juni 2006

\subsection{Akteure}

Deutlich werden die aus der praktizierten Koordinationsform resultierenden unklaren Rollendefinition und -zuschreibungen auch aus der Perspektive einzelner Akteure, die in verschiedener Form in die Steuerung der Kampagne involviert waren:

Der Rat für Forschungs- und Technologieentwicklung hat das Programm initiiert und hält gemäß der Geschäftsordnung den Vorsitz des Programmkomitees. Dieser wird vom Vorsitzenden des Rats für FTE wahrgenommen, der die Sitzungen leitet und das Programmkomitee nach außen vertritt. Im Falle seiner Verhinderung obliegt die Vorsitzführung dem Leiter/der Leiterin der Geschäftsstelle des Rats für FTE. Die Einsicht in die Protokolle der Sitzungen zeigt, dass der Vorsitzende des Rats für FTE nur an der ersten Sitzung teilgenommen hat, und sich in der Folge erst vom Leiter der Geschäftsstelle vertreten ließ, später, nach der Auswahl der Jurysitzung, von dem mit den Agenden des Programms betrauten Mitarbeiter ${ }^{16}$. Diesem fielen dann diverse Aufgaben zu, obwohl der Rat nach Angaben seines Mitarbeiters „weder die Infrastruk-

16 Der Leitung der Geschäftsstelle war in diesem Zeitraum nach einem Personalwechsel nur interimistisch besetzt. 
tur, noch das Geld (hat), um die Koordinations- und Steuerrolle wahrnehmen zu können ${ }^{17 .}$. Er könne „im Prinzip nur über Gespräche agieren“. Tatsächlich stellte sich im Verlaufe der Kampagne heraus, dass das Nebeneinander von Ressortprojekten und gemeinsamen Projekten zu einem „echten Vertragschaos mit vielen Brüchen“ geführt hat, die geglättet werden müssen. Dies umfasst neben Informationsaustausch auch die Verwaltung des Finanzausgleichs bei der Umwidmung von Ressortgeldern für gemeinsame Projekte.

Die Koordination der Initiative wird von verschiedenen Komitees getragen, die sich jeweils aus einer Kerngruppe - dem Rat für FTE, und jeweils einer/m Vertreter/in von BMVIT, BMBWK und BMWA - und weiteren Mitgliedern zusammensetzen. Dem Programmkomitee „obliegt die Generierung eines zielgerichteten Maßnahmen-Katalogs zur Umsetzung der Empfehlung des Rates für FTE vom 25. November 2003. Die Mitglieder des Programmkomitees sorgen weiters für die effektive Umsetzung in den eingebundenen Ressorts und üben Controllingfunktion aus." "s Das Programmkomitee umfasst laut Geschäftsordnung nur die Kerngruppe, und repräsentiert die Auftraggeber/innen. Ab der Beauftragung der FFG mit der Abwicklung der Kampagne ist auch ein/e Vertreter/in der FFG Mitglied des Programmkomitees.

Um eine ausreichende Abstimmung der Koordination mit den Lead-Agenturen zu ermöglichen, wurde als zweite Gruppe ein Lenkungsausschuss eingerichtet, der jene zusätzlich zu Ministerien und FFG einbindet. Der Lenkungsausschuss tagt meist im Anschluss an das Programmkomitee. Schließlich gibt es eine Steuerungsgruppe, bei der neben Rat für FTE und Ministerien auch Science Communications, die für X.PERIMENTA ${ }^{19}$ zuständige Agentur, zugezogen wird, um die Koordination dieses größten Events der Kampagne zu unterstützen. Während die Kooperation zu Beginn der Kampagne zwischen Lead-Agenturen und Organisatoren der Langen Nacht der Forschung - nicht zuletzt aus terminlichen Gründen - noch schwach war, hatten die drei Agenturen über das Jahr Zeit, bis zur X.06 eine kooperative Basis zu finden. Ab Frühjahr 2006 konnten die Sitzungen von Lenkungsausschuss und Steuerungsgruppe dementsprechend zusammengezogen werden.

Alle Mitglieder des Programmkomitees bzw. Lenkungsausschusses beklagen den Aufwand, den diese Sitzungen mit sich bringen, bei gleichzeitig beschränkter Verbindlichkeit bzw. Einhaltung der Beschlüsse. Bereits erwähnt wurde, dass die Besonderheit dieses Komitees darin liegt, Entscheidungen durch die Ressortvertreter/innen ohne weitere Bestätigung durch die Hierarchie der Ressorts treffen zu können. ${ }^{20}$ Ressortintern kann jedoch die Hierarchie, insbesondere Minister/in und sein/ihr Kabinett, trotzdem die stärkere Bindung darstellen, was punktuell zu Widersprüchlichkeiten und einem erneuten Interessensausgleich führen kann. Laut Geschäftsordnung ist „über die Beratungen des Progammkomitees ein Resümeeprotokoll anzufertigen, das in der nächsten Sitzung zur Genehmigung vorzulegen ist." Der Formalismus wurde jedoch nicht immer streng eingehalten, auch wenn Protokolle zu den Sitzungen vorliegen. Um in Zukunft Kritiken zu vermeiden, nach denen einzelne Mitglieder des Programmkomitees sich nicht an Entscheidungen halten, ist es anzuraten, in Zukunft formale Anforderungen einzuhalten, zu Beginn von Sitzungen die Beschlussfähigkeit festzuhalten, und in der Geschäftsordnung festzulegen, nach welchen Zeitspannen ein Protokoll zu legen bzw. zu zeichnen ist.

Bei der Durchführung von Forschungs- und Technologieprogrammen haben in Österreich Agenturen in den vergangenen Jahren durch zunehmende Auslagerung von Programmbetreuung aus den Ministerien an Bedeutung gewonnen, auch kam es durch Zusammenlegungen in nunmehr zwei Agenturen, FFG und AWS, zu einer Konzentration und Strukturierung in der Förderlandschaft. Beide Agenturen sind in unterschiedlichem Ausmaß in die Kampagne innovatives-oesterreich.at involviert. Während die AWS ausschließlich die Ressortprojekte des BMWA verwaltet, wurde die FFG mit der Abwicklung der Ausschreibung der Dachkampagne

\footnotetext{
17 Die Zitate dieses Absatzes beziehen sich auf Interviews, die im Rahmen der Evaluierung durchgeführt wurden.

18 Siehe Geschäftsordnung des Programmkomitees

19 Lange Nacht der Forschung und X.06

20 Den Ressortvertreter/innen obliegt es, diese Entscheidungen im Vorfeld ressortintern abzustimmen.
} 
und der Exekution der Entscheidungen (Verträge, Auflagen, Einforderung von Projektplänen, Finanzen) beauftragt. Ihre Aufgaben beschränken sich somit auf die Programmverwaltung, im Unterschied zur AWS ist die FFG jedoch seit ihrer Beauftragung der Betreuung der Ausschreibung Mitglied des Programmkomitees.

Auf einer anderen Ebene kommt den Lead-Agenturen Trimedia Communications Austria und uma information technology eine koordinierende Rolle zu. Wie in den Terms of Reference der Ausschreibung zu lesen ist, sollen „Modul 1 und Modul 3 der Ausschreibung die kommunikative Klammer für ein breit gefächertes Kommunikations- und Dialogprojekt bilden. Es soll ein flexibler Rahmen entwickelt werden, der die Fülle der Aktivitäten ins öffentliche Bewusstsein hebt, bestehende Kommunikationsleistungen intensiviert und Synergien für die einzelnen Akteure und Angebote nutzbar macht."

Modul 1 der Ausschreibung der Kampagne innovatives-oesterreich.at beinhaltet die „Entwicklung und Durchführung eines integrierten Öffentlichkeitsarbeits- und Kommunikationskonzeptes für das Gesamtprogramm, welches

- die Ziele, Inhalte und Teilprojekte des Programms öffentlichkeitswirksam vermarktet,

- das Gesamtdesign weiterentwickelt

- die Kampagne in den Medien positioniert (Medienkooperationen)

- Elemente von below-the-line Kommunikation entwickelt."

Modul 3 umfasst die „Weiterentwicklung und Anpassung der Informationsdienste (online und Print, etc.)“.

Ursprünglich war geplant, jedes Modul einer Agentur zu übergeben, jedoch zeigte sich während des Auswahlprozesses, dass sich die Jury nicht einigen konnte, welche der in die engere Auswahl gekommenen zwei Agenturen den Zuschlag bekommen sollte. Schließlich fiel die Entscheidung, Modul 1 an beide Agenturen zu vergeben, unter dem Lead von Trimedia, Modul 3 ging an uma. Die beiden Agenturen mussten dementsprechend ihre ursprünglichen Anbote anpassen und einen gemeinsamen Entwurf formulieren, der die Aufgabenteilung bei der Umsetzung der Kampagne wie folgt zusammenfasst: „Trimedia verantwortet die Dachmarke; klassische Werbung, Medienkooperationen und Medienauftritte. Partner für die Gestaltung des Werbeauftritts ist Publicis. uma verantwortet das inhaltliche Konzept der Fragen, die Below-theLine Maßnahmen, Vernetzung mit den anderen Projektträgern sowie Gestaltung und Inhalt der Website." Während das Abfassen eines gemeinsamen Konzepts Vertragsbedingung war, kam es später nicht mehr dazu, dass ein detailliertes Pflichtenheft geschrieben wurde. Es entstand der Eindruck, dass man einen Bauleiter ohne Bauherrn hatte, da der Lenkungsausschuss so ein Pflichtenheft von Trimedia anforderte, jedoch nicht einfordern konnte. Punkte wie klassisches Projektmanagement mit Meilensteinen, Terminen, Zuständigkeiten und Einschätzung der Konsequenzen etwaiger Verschiebungen auf den weiteren Verlauf blieben offen. Die beiden Agenturen sahen ihr Budget-Aufgabenspektrum im Zuge der Zusammenführung gekürzt, und ließen sich nicht auf Zusätzliches ein. Der Wunsch der Auftraggeber/innen, dass die Agenturen ihr eigenes Pflichtenheft festlegen, war nicht vertraglich garantiert, und wohl unter gegebenen Voraussetzungen auch mit den Interessen der beiden Agenturen nicht vereinbar.

Diese Situation wurde auf unterschiedlichen Ebenen als unbefriedigend empfunden: Die LeadAgenturen mussten mit einem Konkurrenten kooperieren, mit gekürztem Budget arbeiten, und sollten zusätzliche Koordinationsaufgaben wahrnehmen, die so nicht von ihnen geplant waren. Der verzögerte Start koordinierter Plattform-Arbeit sowie die Neuorientierung zu stärker zielgruppenorientierten Projekten direkter Interaktion führten dazu, dass die neue Kampagne weniger breite Sichtbarkeit unterstützte, was seitens des Rates für FTE Kritik laut werden ließ. Aus einer Krisensituation, die über mehrere Monate andauerte, zog das Programmkomitee die Konsequenz, einen externen Experten hinzuzuziehen, der als Koordinator für begleitende Maßnahmen ein Budget verwalten sollte, das aus den Mitteln der Lead-Agenturen zweckgewidmet wurde. Ziel war, die vielen kleinen Projekte über die Sichtbarkeitsschwelle zu heben, soweit dies in der verbleibenden Zeit noch möglich war. 


\subsection{Projektaktivitäten im Rahmen der zweiten Kampagnenphase von innovatives-oesterreich.at}

\subsubsection{Projektkategorien}

Die Struktur der Kampagne innovatives-oesterreich.at geht über die einfache Unterscheidung in Projekte/Programme, die von den einzelnen Ressorts verwaltet werden, und gemeinsame Projekte hinaus.

Seitens der Ressortprojekte gibt es in erster Linie jene Initiativen, die unter dem Titel „innovatives-oesterreich.at" gefördert, aber ausschließlich von den Ressorts verwaltet werden. Die Mittelzuteilung zu den jeweiligen Ressorts folgt dem gleichen Schlüssel wie die Aufteilung der gesamten Offensivmittel, nämlich im Verhältnis 44:34:22 für BMVIT, BMBWK und BMWA.

„Unterhalb“ dieser Mittelaufteilung gibt es eine Vereinbarung, manche Projekte unter dem Titel „Poolprojekte“ gemeinsam zu finanzieren, und zwar mit einem Budget, das aus den Ressortmitteln genährt wird, und dies ursprünglich nach dem oben erwähnten Schlüssel, seit dem Frühjahr 2006 von den drei Ressorts zu gleichen Teilen. Bei näherer Betrachtung erkennt man, dass die Poolprojekte zwar einem ressortübergreifenden Interesse dienen, jedoch in der Verwaltung einzelnen Ressorts zuzuordnen sind. Die Poolprojekte werden von Interviewpartner/innen teilweise als eine Art Verhandlungsmasse erwähnt, um durch gegenseitige Unterstützung das eine oder andere Ressort für die Mitfinanzierung größerer Projekte zu gewinnen.

Bei den "gemeinsamen Projekten“ können drei Kategorien unterschieden werden. Einerseits gibt es die X.PERIMENTA, die die Lange Nacht der Forschung 2005 sowie die Wissenschaftsausstellung wahr/falsch inc. umfasst. Wie oben erwähnt wurde dieses Projekt schon frühzeitig lanciert, und nimmt deshalb eine Sonderstellung ein. Andererseits wurde im Februar 2005 die Kampagne innovatives-oesterreich.at mit 4 Modulen ausgeschrieben. Modul 1 und 3 betreffen Dachfunktionen, die an „Lead-Agenturen“ vergeben wurden und im Vergleich zu den weiteren projekt-orientierten Modulen einen besonderen Status haben. Sie umfassen die Entwicklung und Durchführung eines integrierten Öffentlichkeitsarbeits- und Kommunikationskonzeptes für das Gesamtprogramm, Weiterentwicklung und Anpassung der Informationsdienste (Online und Print, etc. Modul 2 beinhaltet die direkte Kommunikation mit relevanten Zielgruppen beispielsweise. in Form von Einzelveranstaltungen und Veranstaltungsreihen, Zielgruppenprojekten, Messebeteiligungen und Ausstellungen. Modul 4 beinhaltet die Entwicklung und Durchführung partizipativer Interaktionsformen zu gesellschaftlich relevanten Themen von Forschung und Innovation.

Schließlich gibt es auch Projekte, die von den Sozialpartnern initiiert wurden. Während in der ersten Kampagne die Sozialpartner Mitglieder des Lenkungsausschusses waren, sind sie das in der zweiten Kampagne nicht mehr. Im Jänner 2005 wurde beschlossen, dass die Sozialpartner ${ }^{21}$ bis zu $65.000 €$ für Projekte im Rahmen von innovatives-oesterreich.at bekommen. Die Förderung der Projekte der Industriellenvertretung wurde, mit Ausnahme des Projektes der IV Salzburg, vom BMVIT übernommen, das BMWA übernahm wie schon zuvor die Abwicklung der Sozialpartnerprojekte.

\subsubsection{Die einzelnen Projekte - Projekttypen, Zielgruppen und Mobilisierungswege}

Im Rahmen der Kampagne innovatives-oesterreich.at wurden über 50 Projekte durchgeführt. Thematisch reicht das Spektrum der Aktivitäten von der Kinderuniversität über den Erfinderpreis „Jugend Innovativ“, einen Fotowettbewerb, Ausstellungen und Diskussionen bis hin zu dem Großereignis "Lange Nacht der Forschung" (vgl. die Aufstellung in Tabelle 3 sowie die Einzeldarstellungen der Projekte im Anhang). Die Spannbreite der Projektbudgets reicht von $18.000 €$ bis hin zu 1,26 Mio $€$. Insgesamt konnten mit den diversen Projektaktivitäten mindestens 267.500 Besucher/innen bzw. Teilnehmer/innen erreicht werden.

21 AK, ÖGB, WKÖ, IV Landesorganisationen 
Tabelle 3 Projekte im Rahmen der 2. Kampagne innovatives-oesterreich.at

\begin{tabular}{|c|c|c|c|}
\hline Projekttitel & Projektträger & $\begin{array}{l}\text { Laufzeit/ } \\
\text { Zeitpunkt }\end{array}$ & $\begin{array}{l}\text { Besucher- } \\
\text { zahl }\end{array}$ \\
\hline \multicolumn{4}{|c|}{ Ausstellungen / Konferenzen } \\
\hline Age of Simulation & AEC ARS Ars Electronica Center Linz & 02/2006-08/2006 & k.A. \\
\hline die wahr/falsch inc. & science communications & $06 / 2006-07 / 2006$ & 13.600 \\
\hline Gender\&Science & science communications & 02/2005-03/2005 & 100 \\
\hline Gödels Jahrhundert & $\begin{array}{l}\text { Österreichische Mathematische } \\
\text { Gesellschaft }\end{array}$ & 04/2006-08/2006 & 3.500 \\
\hline Language of Networks & AEC ARS Ars Electronica Center Linz & $09 / 2004$ & 144.000 \\
\hline SCOPE-Konferenz & uma information technology & $09 / 2006$ & 113 \\
\hline $\begin{array}{l}\text { Transatlantic Dicord. } \\
\text { Combating Terrorism and } \\
\text { Proliferation, preventing } \\
\text { Crisis }\end{array}$ & ÖGPW & $12 / 2005$ & 80 \\
\hline \multicolumn{4}{|c|}{ Dialog im öffentlichen Raum } \\
\hline Forschungstage 2006 & bmvit & $09 / 2006$ & k.A. \\
\hline $\begin{array}{l}\text { Lange Nacht der } \\
\text { Forschung }\end{array}$ & science communications & $10 / 2005$ & 48.000 \\
\hline $\begin{array}{l}\text { Club Research - Open } \\
\text { House der Forschung }\end{array}$ & doppio espresso & 02/2006-12/2006 & 180 \\
\hline $\begin{array}{l}\text { Public Understanding of } \\
\text { Life Sciences }\end{array}$ & Dialog Gentechnik & $2005-2006$ & k.A. \\
\hline Stand des Wissens & Plansinn & 05/2006-10/2006 & $\begin{array}{c}1.600 \\
\text { Dialoge }\end{array}$ \\
\hline \multicolumn{4}{|c|}{ Fachveranstaltungen } \\
\hline Forschung für Uns & $\begin{array}{l}\text { PR\&D Public Relations for Research and } \\
\text { Development }\end{array}$ & 08/2005-12/2006 & 250 \\
\hline High Tech - Low Quality & science communications & $10 / 2005-01 / 2006$ & 265 \\
\hline $\begin{array}{l}\text { International Networking } \\
\text { for Young Scientists }\end{array}$ & British Council & $02 / 2005$ & k.A. \\
\hline $\begin{array}{l}\text { Kommunikationswissen- } \\
\text { schaftliche Tage }\end{array}$ & Institut für Technikabfolgenschätzung & $11 / 2004$ & 115 \\
\hline Mapping Controversities & ÖAW & $01 / 2005$ & 100 \\
\hline Techpol 2.0 & Institut für Technikabfolgenschätzung & $09 / 2005-06 / 2006$ & k.A. \\
\hline \multicolumn{4}{|c|}{ Kinder- und Jugendprogramm } \\
\hline Forschung mit Fun & $\begin{array}{l}\text { PR\&D Public Relations for Research and } \\
\text { Development }\end{array}$ & $10 / 2005-12 / 2006$ & 466 \\
\hline Junge Uni Innsbruck & Universität Innsbruck & 2004-2006 & 15.000 \\
\hline Junge Uni Krems & IMC FH Krems & 2006 & 200 \\
\hline Kinderuni Steyr & IFAU Institut für Angewandte Umweltbildung & $2005-2006$ & k.A. \\
\hline Kinderuni Wien & Universität Wien & 2004-2006 & 4.000 \\
\hline Lehre und Forschung & Plansinn & 05/2006-12/2006 & k.A. \\
\hline $\begin{array}{l}\text { Tage der jungen } \\
\text { Forschung }\end{array}$ & communication matters & $11 / 2005$ & 83 \\
\hline ZOOM Science & $\begin{array}{l}\text { ZOOM-Kindermuseum - Verein Interaktives } \\
\text { Kindermuseum }\end{array}$ & 09/2005-03/2006 & 25.700 \\
\hline $\begin{array}{l}\text { Schlaufuchsakademie } \\
\text { Ennstal }\end{array}$ & IFAU Institut für Angewandte Umweltbildung & 2004-2006 & k.A. \\
\hline
\end{tabular}




\begin{tabular}{|c|c|c|c|}
\hline Projekttitel & Projektträger & $\begin{array}{l}\text { Laufzeit/ } \\
\text { Zeitpunkt }\end{array}$ & $\begin{array}{l}\text { Besucher- } \\
\text { zahl }\end{array}$ \\
\hline \multicolumn{4}{|c|}{ Stärkung der Wissenschaftskommunikation } \\
\hline $\begin{array}{l}\text { Medienkooperation im } \\
\text { Wettbewerb }\end{array}$ & doppio espresso & $12 / 2005-09 / 2006$ & 150 \\
\hline Meet the Media & communication matters & $11 / 2005-12 / 2006$ & 250 \\
\hline Pressereise Schweden & communication matters & 09/2005-06/2006 & k.A. \\
\hline $\begin{array}{l}\text { SciMedia - } \\
\text { Universitätslehrgang } \\
\text { Wissenschaftskommunikati } \\
\text { on }\end{array}$ & $\begin{array}{l}\text { IFF - Fakultät für Interdisziplinäre } \\
\text { Forschung und Fortbildung }\end{array}$ & $2005-2006$ & 16 \\
\hline \multicolumn{4}{|c|}{ Projekte der Sozialpartner } \\
\hline $\begin{array}{l}\text { Arbeitsplätze durch } \\
\text { Innovation }\end{array}$ & AK Wien & 01/2005-05/2006 & $\begin{array}{c}110 \\
\text { (Bundessie } \\
\text { gerehrung) } \\
\end{array}$ \\
\hline Frauen\&Technik & IV-Salzburg & k.A. & k.A. \\
\hline Innovation V & IV-Vorarlberg & $2005-2006$ & 2005: 600 \\
\hline Innovationsgespräche & IV-Steiermark (inno regio) & 2005 & 150 \\
\hline Innovationstag & IV-Salzburg & $11 / 2005$ & k.A. \\
\hline Innovations-Updates & IV-Salzburg & $10 / 2005-03 / 2006$ & k.A. \\
\hline innovatives-unternehmen & WIFI, WKÖ & 01/2006-12/2006 & 5.000 \\
\hline iScan & IV-Steiermark (inno regio) & $10 / 2005$ & k.A. \\
\hline Jobmotor Forschung & GPA & $12 / 2005-09 / 2006$ & k.A. \\
\hline Kreatives Kärnten & IV-Kärnten & $09 / 2005-06 / 2006$ & k.A. \\
\hline \multicolumn{4}{|c|}{ Auszeichnungen, Preise und Wettbewerbe } \\
\hline $\begin{array}{l}\text { Fotowettbewerb } \\
\text { Innovative Motive }\end{array}$ & doppio espresso & $11 / 2005-02 / 2006$ & 250 \\
\hline ideenreich.at & Dr. Hochegger Kommunikationsberatung & $2003-2005$ & 780 \\
\hline Jugend Innovativ & aws - Austria Wirtschaftsservice & $2005-2006$ & 1.069 \\
\hline Staatspreis Innovation & aws - Austria Wirtschaftsservice & 2004-2006 & $\begin{array}{c}1.062 \\
(2004 \\
2005)\end{array}$ \\
\hline Staatspreis Multimedia & ICNM - International Center for New Media & $2004-2006$ & \begin{tabular}{|l}
600 \\
(Veranstalt \\
ung), 206 \\
Teilnehmer \\
/innen \\
\end{tabular} \\
\hline \multicolumn{4}{|c|}{ Produkte } \\
\hline applied research in a box & Communication matters & k.A. & k.A. \\
\hline $\begin{array}{l}\text { at.venture - Magazin zu } \\
\text { Forschung, Technologie } \\
\text { und Innovation }\end{array}$ & LW-Media Verlag & seit 09/2006 & k.A. \\
\hline Quintessenz & Z.ONE-media & 08/2005-10/2006 & k.A. \\
\hline $\begin{array}{l}\text { sclQ - Wissen für junge } \\
\text { Leute }\end{array}$ & $\operatorname{sclQ}$ & k.A. & k.A. \\
\hline technology log & permalink & $2005-2006$ & k.A. \\
\hline \multicolumn{4}{|c|}{ Dachkampagne } \\
\hline $\begin{array}{l}\text { Dachkampagne } \\
\text { innovatives-oesterreich.at: } \\
\text { Internetportal u. } \\
\text { Fragenkampagne }\end{array}$ & uma information technology & 06/2005-12/2006 & \\
\hline $\begin{array}{l}\text { Dachkampagne } \\
\text { innovatives-oesterreich.at: } \\
\text { Werbung, PR }\end{array}$ & Trimedia & 06/2005-12/2006 & \\
\hline \multicolumn{4}{|c|}{ Besucheranzahl gesamt: 267.595} \\
\hline
\end{tabular}


Differenziert nach Veranstaltungsart bzw. Projektkategorie waren „punktuelle Veranstaltungen zu mehreren Terminen" am stärksten vertreten. Die Hälfte aller befragten Projektträger/innen ordneten ihr Projekt bzw. ihre Projekte dieser Kategorie zu, gefolgt von der Kategorie „Medienkooperation“ mit 12 Nennungen (Grafik 7). Jeweils neun der Befragten gaben weiters an, ihr Projekt bzw. ihre Projekte zählen zu den Kategorien „einmalige Veranstaltungen“, „Wettbewerb/Preise/Awards“ sowie "Serviceleistung“. Acht Projektträger/innen ordneten ihr Projekt bzw. ihre Projekte der Kategorie „Veranstaltungen über längeren Zeitraum“ zu, lediglich zwei der Kategorie „Qualifizierungsprojekt“.

Grafik 7 Projekte im Rahmen der Kampagne innovatives-oesterreich.at nach Projektkategorien

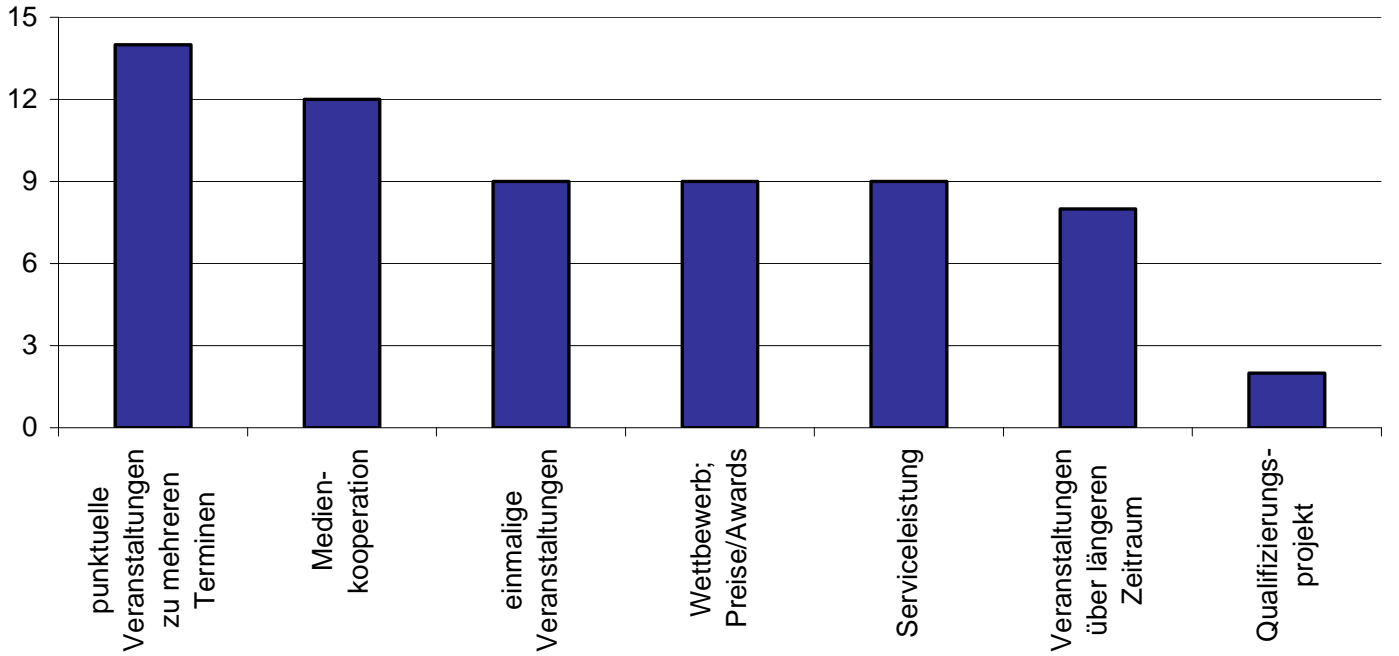

Anmerkungen:

$\mathrm{N}=25$, Mehrfachantworten möglich

Anzahl der Nennungen auf die Frage: „Welcher der folgenden Kategorien können Ihre Projektaktivitäten zugeordnet werden?"

Quelle: KMU FORSCHUNG AUSTRIA, Online-Befragung Frühjahr 2006 
Betrachtet man die Angaben über die durch die Projektaktivitäten angesprochenen Zielgruppen, so werden in erster Linie die Gruppen „Wissenschafts- und Forschungsszene“, „Kinder/Jugendliche“ und „Medien/Journalisten“ mit jeweils 17 Angaben, gefolgt von der Gruppe „Entscheidungsträger" (16) adressiert (Grafik 8). 13 Projektträger/innen geben an, im Besonderen die Gruppe „KMU“ ansprechen zu wollen, hingegen beabsichtigen nur sechs, speziell Großunternehmen zu adressieren. Die Gruppe „Frauen“ ist für neun Projektträger/innen vorrangige Zielgruppe.

\section{Grafik $8 \quad$ Zielgruppen der Projektaktivitäten}

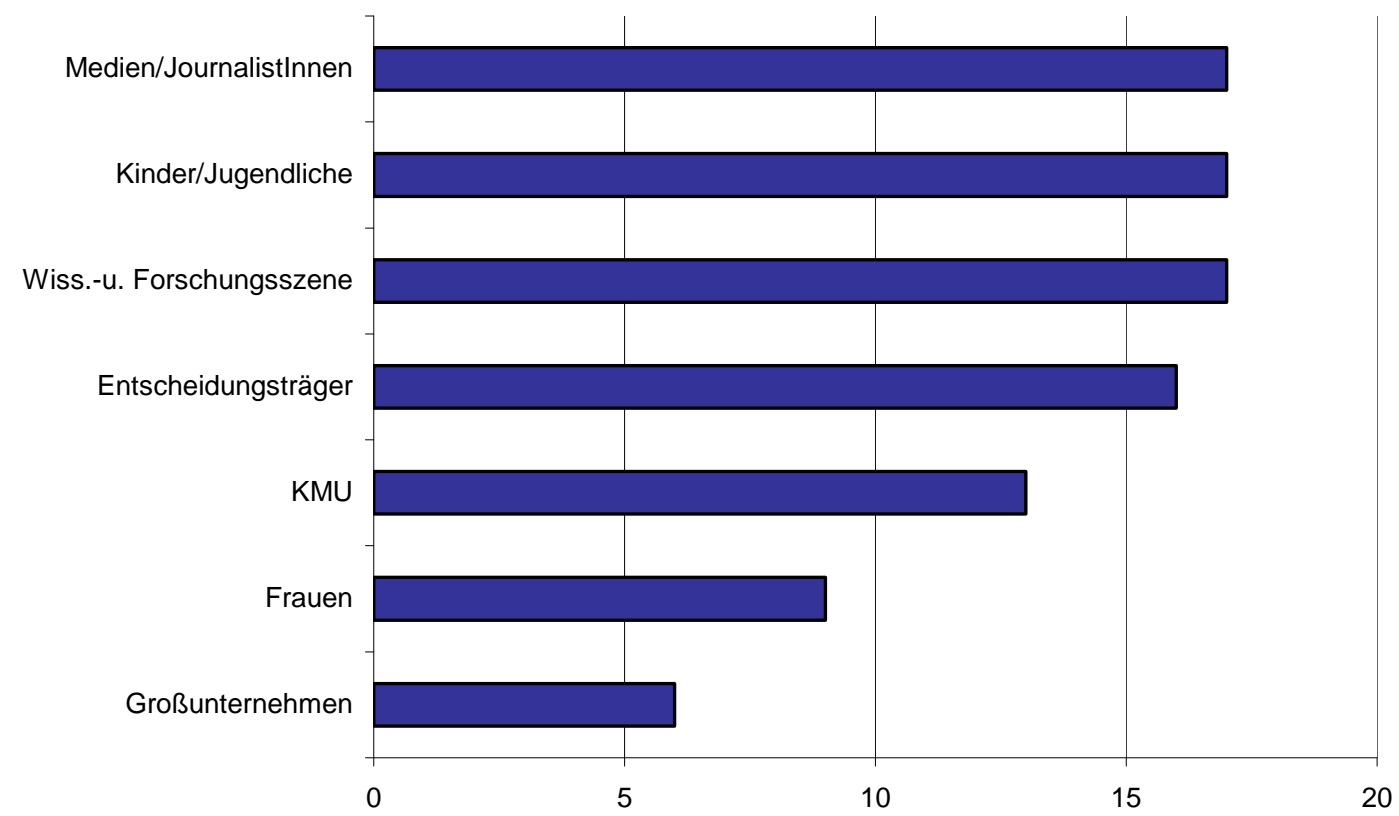

Anmerkungen:

$\mathrm{N}=27$, Mehrfachantworten möglich

Anzahl der Nennungen auf die Frage: „Welche der folgenden Gruppen soll mit Ihren Projektaktivitäten insbesondere angesprochen werden?"

Quelle: KMU FORSCHUNG AUSTRIA, Online-Befragung Frühjahr 2006 
Bei den zum Zeitpunkt der Befragung im Frühjahr 2006 bereits „absolvierten“ Projektaktivitäten - dies trifft nach Angaben der Projektträger/innen auf 20 Projekte zu - waren Interessierte mit einschlägigen Vorkenntnissen, Repräsentanten der Medienöffentlichkeit, Fachpublikum und Schüler/innen bzw. Jugendliche insgesamt am stärksten vertreten (Grafik 9). Die am schwächsten vertretene Besuchergruppe sind laut Angaben der Projektträger/innen Familien mit Kindern.

Grafik $9 \quad$ Publikum der Projektaktivitäten im Rahmen der Kampagne

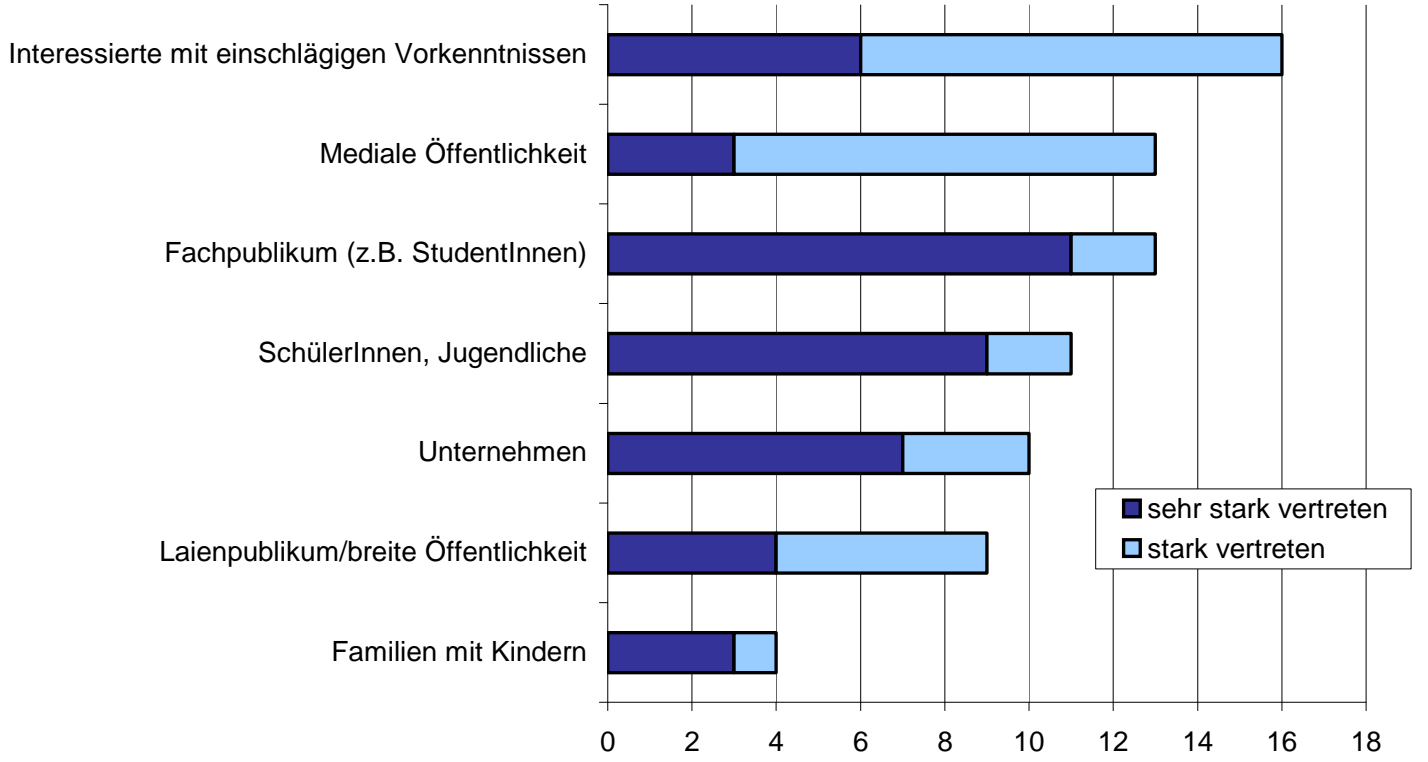

Anmerkungen:

$\mathrm{N}=14-19$

Anzahl der Antworten der Projektträger von zum Zeitpunkt der Befragung bereits stattgefundenen Projektaktivitäten; „sehr stark vertreten“ bzw. „stark vertreten“ auf die Frage: „Welche Publikum hat Ihre Projektaktivitäten besucht?“

Quelle: KMU FORSCHUNG AUSTRIA, Online-Befragung Frühjahr 2006 
Mobilisiert wurde bzw. wird für die Veranstaltungen - in absteigender Rangfolge - über E-MailAussendungen, Internet, Plakate und Flyer, postalische Aussendungen, Inserate in Printmedien und schließlich über Schaltungen in Radio und Fernsehen (Grafik 10).

Grafik 10 Reale und potenzielle Mobilisierungswege für Projektaktivitäten im Rahmen der Kampagne

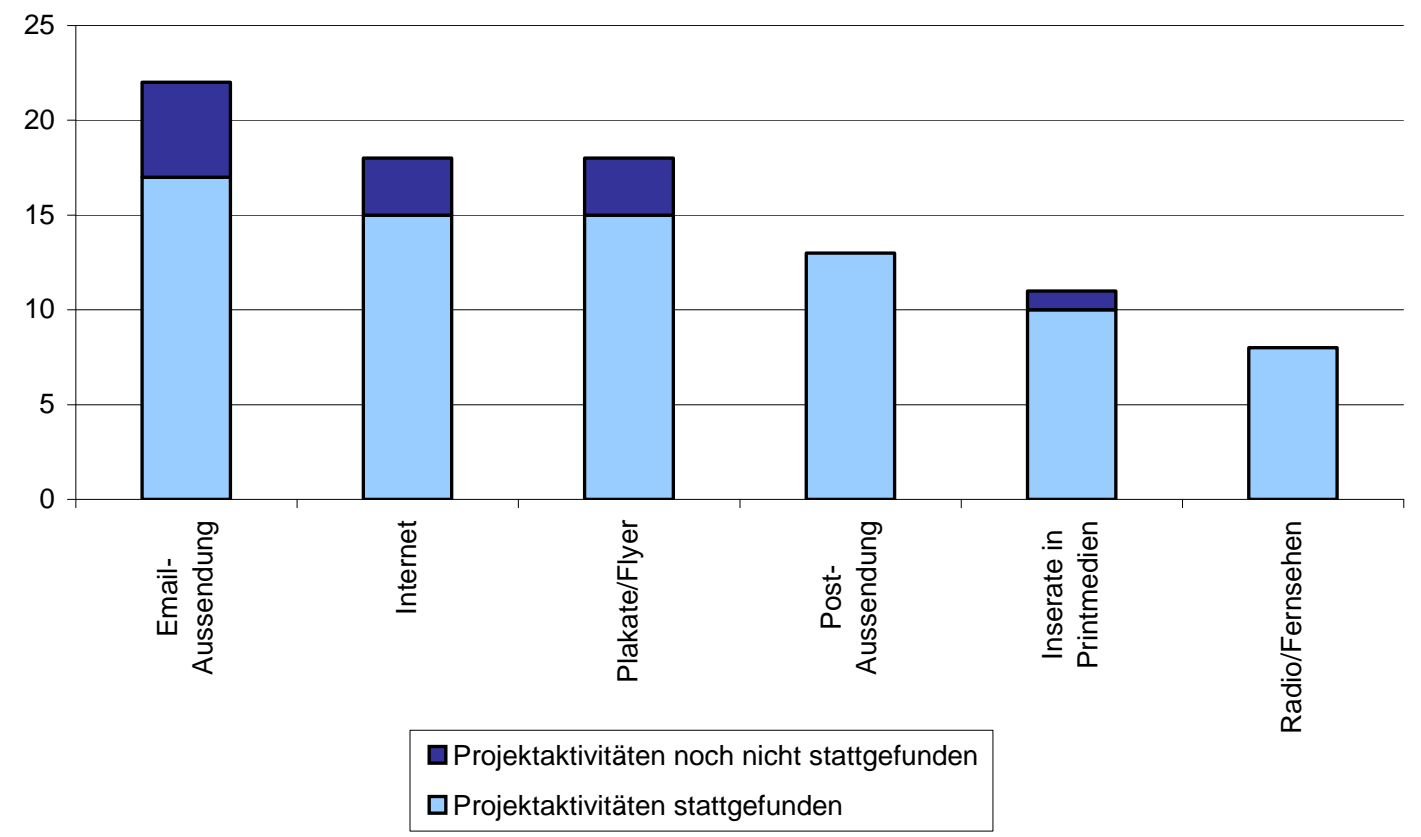

Anmerkungen:

$\mathrm{N}=20$ bzw. 8; Mehrfachantworten möglich

Anzahl der Nennungen auf die Frage: „Auf welchem Weg fand bei Ihren Aktivitäten im Rahmen von innovativesoesterreich.at die Mobilisierung der Zielgruppen statt bzw. auf welchem Weg soll diese stattfinden?"

Quelle: KMU FORSCHUNG AUSTRIA, Online-Befragung Frühjahr 2006 



\section{$4 \quad$ Prozesse und Steuerung}

Für den Erfolg der Kampagne sowie für Steuerung und die im Rahmen von innovativesoesterreich.at ablaufenden Prozesse ist nicht zuletzt die Motivation, die der Teilnahme der Projektträger an der Kampagne zugrunde liegt (4.1), von Bedeutung. Die für die Kampagne innovatives-oesterreich.at relevanten Prozesse basieren zudem auf der Auswahl der Projekte (4.2), dem Programmmanagement (4.3) und Community-Building (4.4). Von Bedeutung ist im Sinne der Realisierung von Lerneffekten für die Steuerung derartiger Kampagnen das beobachtete organisationale Lernen, das heißt die im Verlaufe der zweiten Kampagnenphase notwendigen und vorgenommenen Zielanpassungen sowie die Bestellung eines Projektmanagers bzw. Medienkoordinators (4.5).

\subsection{Motivation der Projektträger}

Als vorrangige Motivation für die Teilnahme an der Kampagne werden die Kommunikation von Wissenschaft und Forschung nach außen sowie der Beitrag zur Akzeptanzsteigerung von Wissenschaft und Forschung gesehen. Für 20 befragte Projektträger/innen ist das Motiv der Wissenschafts- und Forschungskommunikation "sehr wichtig“, für weitere fünf "wichtig“ (Grafik 11). Einen Beitrag zur Steigerung gesellschaftlicher Akzeptanz von Wissenschaft und Forschung zu leisten ist für 19 Befragte „sehr wichtig“, fünf sehen dieses Motiv als „wichtig“ an. Eine Deckung der Kampagnenzielsetzungen mit ihrem Alltags- bzw. Kerngeschäft gilt für 5 bzw. 12 der Projektträger/innen als sehr wichtiges bzw. wichtiges Motiv. Reines Interesse in diesem Bereich zu arbeiten war für fünf ein sehr wichtiger und für weitere 13 ein wichtiger Teilnahmegrund. Im Vergleich zu den genannten Teilnahmegründen ist der der Rekrutierung von potenziellem Forschungsnachwuchs ein weniger häufig genanntes Motiv: jeweils fünf der befragten Projektträger/innen geben an, dieser Aspekt sei „sehr wichtig“ bzw. „wichtig“. Finanzielle Gründe haben für 16 eine zumindest wichtige Rolle gespielt.

Grafik $11 \quad$ Motive für die Teilnahme an der Kampagne

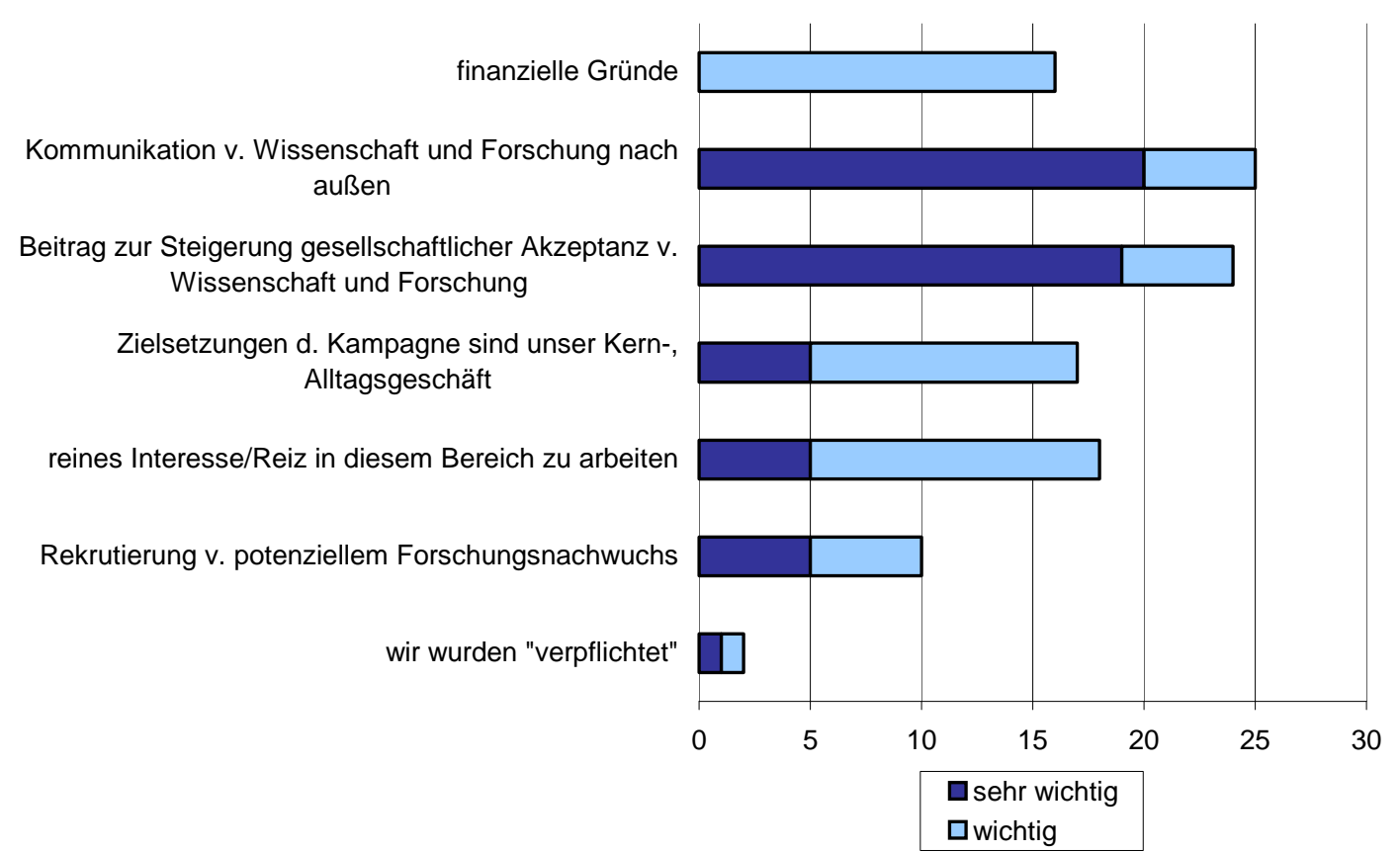

Anmerkungen: $\mathrm{N}=22-26$

Anteil der Antworten „sehr wichtig“ und „wichtig“ auf die Frage: „Welche Motive haben Sie für die Teilnahme an der Kampagne innovatives-oesterreich.at?"

Quelle: KMU FORSCHUNG AUSTRIA, Online-Befragung Frühjahr 2006 


\subsection{Auswahl der Projekte}

Je nach Projekttyp bzw. Finanzierung kamen unterschiedliche Auswahlprozesse zum Tragen. Ressortprojekte wurden direkt von den zuständigen Ministerien nach klassischen Vergaberegeln vergeben. Gemeinsame Projekte wurden zum Großteil im Rahmen der Ausschreibung der Kampagne vergeben, eine Ausnahme bildet die X.PERIMENTA, die nach einem Ideenwettbewerb vergeben wurde.

Mit der Ausschreibung der Kampagne wurde ein - im Vergleich zur ersten Kampagnenphase wesentlicher Schritt zu mehr Transparenz gesetzt. Die Vorbereitung der Ausschreibung oblag dem Programmkomitee unter der Leitung des Rats für FTE, nach Angaben aller Beteiligten waren zahlreiche Besprechungen notwendig, um eine gemeinsame Position zu finden. Die Auswahl erfolgte nach einem zweistufigen Verfahren (Bewerbung und schriftlicher Antrag), und einem Hearing der Bewerber/innen für Modul 1. Antragsfrist war der 15. März 2005, Hearings fanden im April 2005 statt. Insgesamt wurden, wie bereits dargerstellt (vgl. Kap. 3.3.1), vier Module ausgeschrieben. Im Jänner 2005 wurde die FFG mit der Ausschreibung und Abwicklung des Programms beauftragt, das heißt zu einem Zeitpunkt, als der Ausschreibungstext schon feststand. Insgesamt war die Ausschreibung sehr erfolgreich: Es wurden drei- bis viermal so viele Anträge eingereicht wie erwartet, das geplante Volumen war vier- bis sechsfach überzeichnet.

Die Jury tagte dreimal; sie setzte sich aus neun Mitgliedern zusammen: dem Vorsitzende des Rats für FTE (teilweise durch den Leiter der Geschäftsstelle vertreten), den Vertreter/innen der drei Ressorts, sowie fünf externen Expert/innen, von denen jeweils einer von den beteiligten Ressorts und dem Rat benannt wurde (ein Vertreter der Bundeswirtschaftskammer Österreich (BWK), des ORF, eine ehemalige Pressesprecherin des BMVIT, sowie ein unabhängiger Publizist). Der neunte Experte, ein Vertreter des Bundeskanzleramts, konnte nicht an den JurySitzungen teilnehmen und ließ sich auch nur bei der ersten Sitzung vertreten - ein Umstand, der dazu führte, dass gerade in der sehr komplizierten Abstimmung über die Lead-Agentur Stimmengleichheit bestand und eine Kompromisslösung gefunden werden musste (vgl.Kap.3.2). Bezüglich des Abstimmungsverhaltens wurde berichtet, dass die externen Expert/innen zwar teilweise aber nicht ausschließlich mit den sie benennenden Ressorts „mitstimmten", eine Unabhängigkeit schien also gewährleistet zu sein.

Die Auswahl der Projekte der Module 2 und 4 erfolgte auf Basis schriftlicher Bewertungen durch die Jurymitglieder. Die Bewerber/innen für Modul 1 und 3 wurden einem Hearing unterzogen. Dabei kam es zu unvorhergesehenen Komplikationen, da sich die Jury nicht auf einen Kandidaten einigen konnte. Nach einem mehrstufigen Abstimmungsprozess wählte man als Kompromisslösung die Vergabe an die zwei bestgereihten Agenturen, von denen die eine insbesondere durch ihr inhaltliches Konzept, die andere durch ihre Kompetenz in PR und Medienarbeit überzeugte. Dieses vom Auftraggeber zusammengesetzte Konsortium wurde aufgefordert, als Vertragsgrundlage ein gemeinsames Konzept auf Basis definierter Auflagen zu erstellen. Insgesamt verzögerte diese Konstruktion den Start des Programms teilweise, da die Agenturen nicht unmittelbar starten konnten, sondern ihre Pläne erst an die neuen Bedingungen anpassen mussten.

Das größte Projekt im Rahmen von innovatives-oesterreich.at ist die X.PERIMENTA (X.06), das sich aus zwei Pilotprojekten zusammensetzt: der Langen Nacht der Forschung 2005 und der WAHR/ FALSCH INC., einer Wissenschaftsausstellung in Wien. Die Auswahl dieses Projekts hatte eine längere Vorlaufzeit. Im Frühjahr 2003 wurde ein Ideenwettbewerb unter dem Titel „Fest der Forschung“ vom BMVIT ausgeschrieben. Im September 2003 wurde die Agentur Science Communications vom Gewinn des 1. Preises informiert, welcher der Agentur im März 2004 (!) im Rahmen einer Festveranstaltung im Parlament symbolisch übergeben wird. Währenddessen war Science Communications damit beauftragt worden, ein Konzept für die Umsetzung eines Events zu entwickeln, das den Grundstein für die Lange Nacht der Forschung und X.06 setzte. Die lange Vorlaufzeit erklärt sich durch die Vorbereitung der zweiten Laufzeit von innovatives-oesterreich.at, im Zuge dieser das geplante Event von dem Programm aufge- 
griffen wurde. Als im August 2004 die Gesamtmittel für die Kampagne fixiert wurden, wurde auch ein ressortübergreifendes Budget an das Fest der Forschung (späterhin X.PERIMENTA) gebunden. Die schriftliche Beauftragung erfolgte im Dezember 2004 durch das BMVIT, mit der Abwicklung der X.PERIMENTA wurde ab Jänner 2005 die FFG betraut. Es lässt sich also in diesem Fall ein Übergang von einer Ressortinitiative zu einem gemeinsamen Projekt beobachten, der eine Projektgröße erlaubt, die von einem Ressort alleine nicht hätte finanziert werden können. Weiters erkennt man aber auch, dass von den Auftragnehmern finanziell ein besonders langer Atem erwartet wurde. Auch sind die unterschiedlichen Ausschreibungsbedingungen für die Kampagne einerseits und das Event andererseits hervorzuheben (öffentliche Ausschreibung eines Gesamt-Kampagnenbudgets nach Modulen und schrittweises Wachsen eines Großprojektes auf Basis des 1. Preises bei einem Ideenwettbewerb). Schließlich erlaubte das Timing von Event (zuerst) und Kampagne mit Lead-Agentur (später) nicht, dass die Lange Nacht der Forschung zur Gänze unter dem Dach innovatives-oesterreich.at stattfand; dieses „Großereignis" der zweiten Kampagnenphase wurde daher auch nur bedingt als "Ereignis“ von innovatives-oesterreich.at wahrgenommen.

\subsection{Programmmanagement - die Sicht der Projektträger}

Es wurde bereits darauf hingewiesen, dass die Koordination der zweiten Kampagnenphase und die Zuordnung bestimmter Steuerungsaufgaben zu beteiligten Akteuren zunächst durchaus kritisch zu bewerten war (vgl. Kapitel 3.1). Diese Sichtweise spiegelt sich in Teilaspekten auch in der Bewertung des Programmmanagements durch die Projektträger/innen wider. Gleichwohl ist zunächst bemerkenswert, dass bei den Projektträger/innen eine gewisse „Grundzufriedenheit" mit der Teilnahme an der Kampagne zu überwiegen scheint. So betrachten 25 der 27 befragten Projektträger/innen den für die Teilnahme an der Kampagne erforderlichen Aufwand als angemessen. Dass 25 Projektträger/innen angeben, sie hätten ihre Projekte ohne nennenswerte zeitliche Verzögerungen durchgeführt, spricht ebenfalls dafür, dass es keine übermäßigen, administrativ bedingten Einschränkungen der Projektaktivitäten gab. In dieses Bild fügt sich, dass 16 Befragte ihre Erwartungen an die Kampagne als „erfüllt“ ansehen, drei als „übertroffen" und lediglich vier als "nicht erfüllt". Dementsprechend hoch ist auch das geäußerte Interesse an Folgeeinreichungen bei einer eventuell anstehenden weiteren Phase der Kampagne innovatives-oesterreich.at: 21 Projektträger/innen würden „auf jeden Fall“ nochmals ein Projekt im Rahmen von innovatives-oesterreich.at einreichen, drei können sich eine abermalige Einreichung unter anderen Bedingungen vorstellen (Grafik 12). Genannt werden hier die Berücksichtigung aller Projekte bei den kampagnenbezogenen PR-Aktivitäten sowie klare Vorgaben des Kampagnen- und Projektablaufs durch den Auftraggeber. 


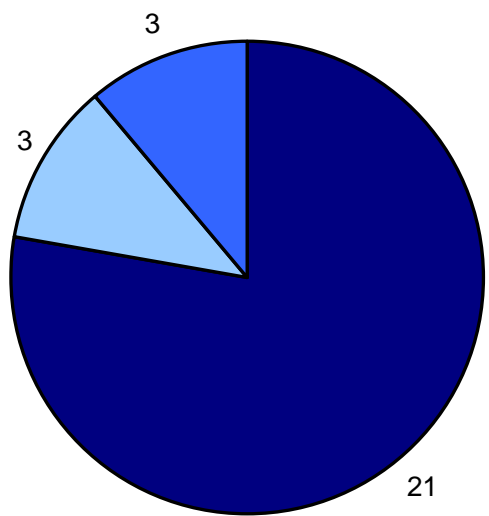

aja, auf jeden Fall

口ja, aber unter anderen Umständen/Bedingungen

$\square$ weiß nicht

Anmerkungen:

$\mathrm{N}=27$

Anteil der Antworten auf die Frage: „Würden Sie nochmals ein Projekt im Rahmen von innovatives-oesterreich.at einreichen?"

Quelle: KMU FORSCHUNG AUSTRIA, Online-Befragung Frühjahr 2006

Diese „Grundzufriedenheit“ der Projektträger/innen mit der Kampagne innovativesoesterreich.at kann allerdings nicht über einige kritische Punkte hinwegtäuschen, die nicht zuletzt in Zusammenhang mit der angesprochenen defizitären Koordination der Kampagne stehen. So zeigt die nähere Betrachtung der Bewertung des Programmmanagements durch die Projektträger/innen zwar, dass sowohl die Höhe der Förderung als auch einzelne administrative Aspekte wie die Vertragsgestaltung oder der administrative Aufwand für die Projektabwicklung durchaus gut bewertet werden (Grafik 13). Nur noch durchschnittlich fällt dann allerdings die Bewertung der Unterstützung bei Vorbereitung und Durchführung der Projekte aus, hier haben sich zumindest einzelne Projektträger/innen, wie auch in den Interviews zum Ausdruck kam, offenkundig mehr Unterstützung erwartet. Genannt werden hier insbesondere die vermisste Unterstützung bei der Mobilisierung zur Veranstaltung sowie die Medienarbeit. In diesem Kontext steht insbesondere das lediglich „befriedigend“ in der Bewertung der Arbeit der Lead-Agenturen Trimedia und uma. ${ }^{22}$

22 Dass Projektauswahlprozess und die Verteilung der Finanzmittel zwischen den Projekten von Förderwerbern eher kritisch bewertet werden, kann als „Normalfall“ angesehen werden. 


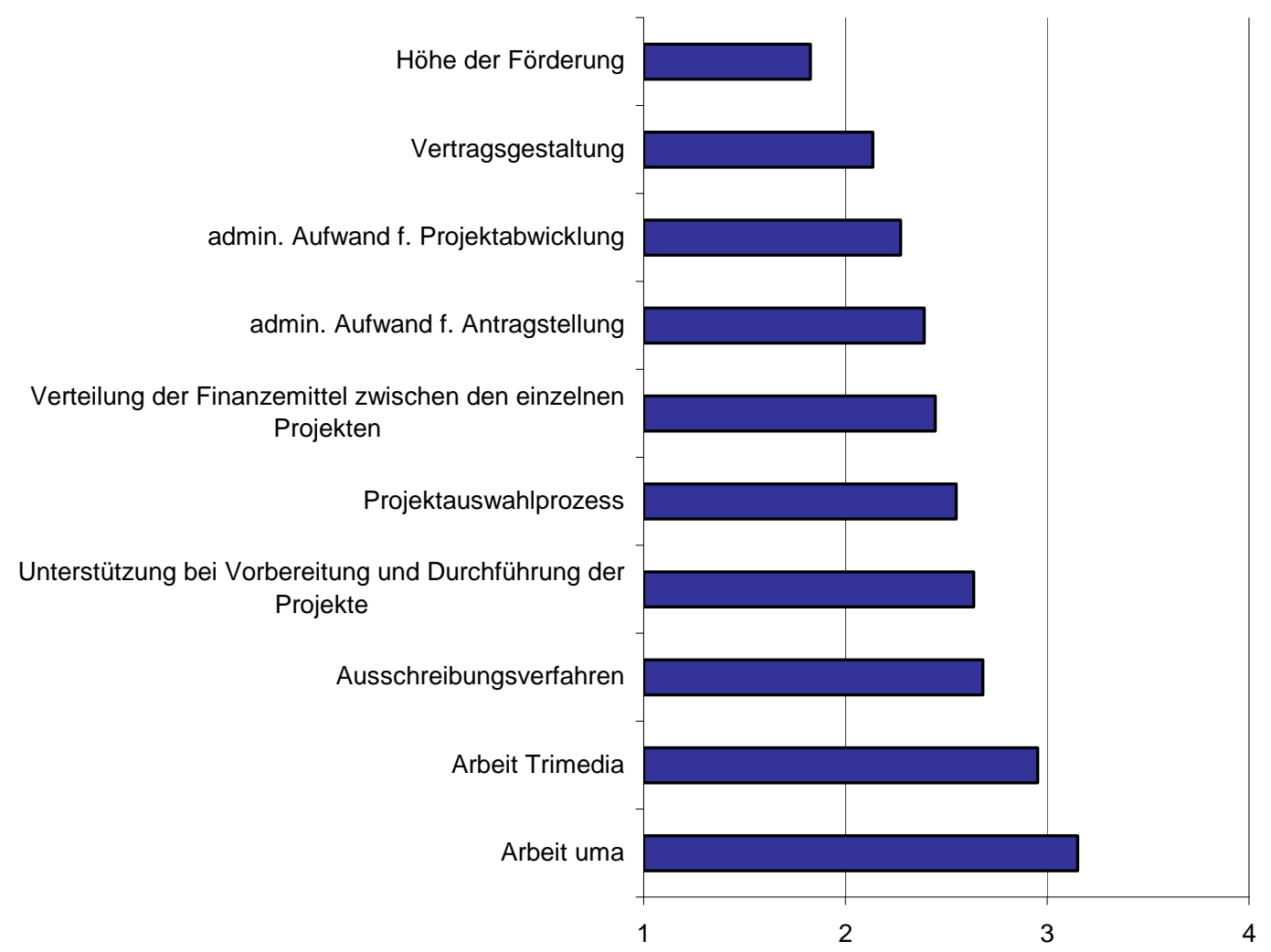

Anmerkungen:

$\mathrm{N}=18-23$

Bewertungen auf die Frage: „Wie bewerten Sie folgende Aspekte der Steuerung und praktischen Umsetzung der Kampagne innovatives-oesterreich.at." Mittelwerte der Bewertungen nach Schulnoten von 1 = sehr gut" bis $5=$ nicht genügend.

Quelle: KMU FORSCHUNG AUSTRIA, Online-Befragung Frühjahr 2006

Hinsichtlich der kampagnenbezogenen PR-Arbeit existiert aus Sicht der Projektträger/innen offenkundig Verbesserungspotenzial (Grafik 14). Dies betrifft, wie erwähnt, insbesondere die Veranstaltungsmobilisierung, aber auch die Medien- und Pressearbeit. Die Integration der Dachmarke, deren Bedeutung von den Projektträger/innen für den Kampagnenerfolg als durchaus hoch eingeschätzt wird, sowie der Web-Auftritt werden hingegen als vergleichsweise zufriedenstellend bewertet; weniger Anklang fand allerdings die Fragenbank. 


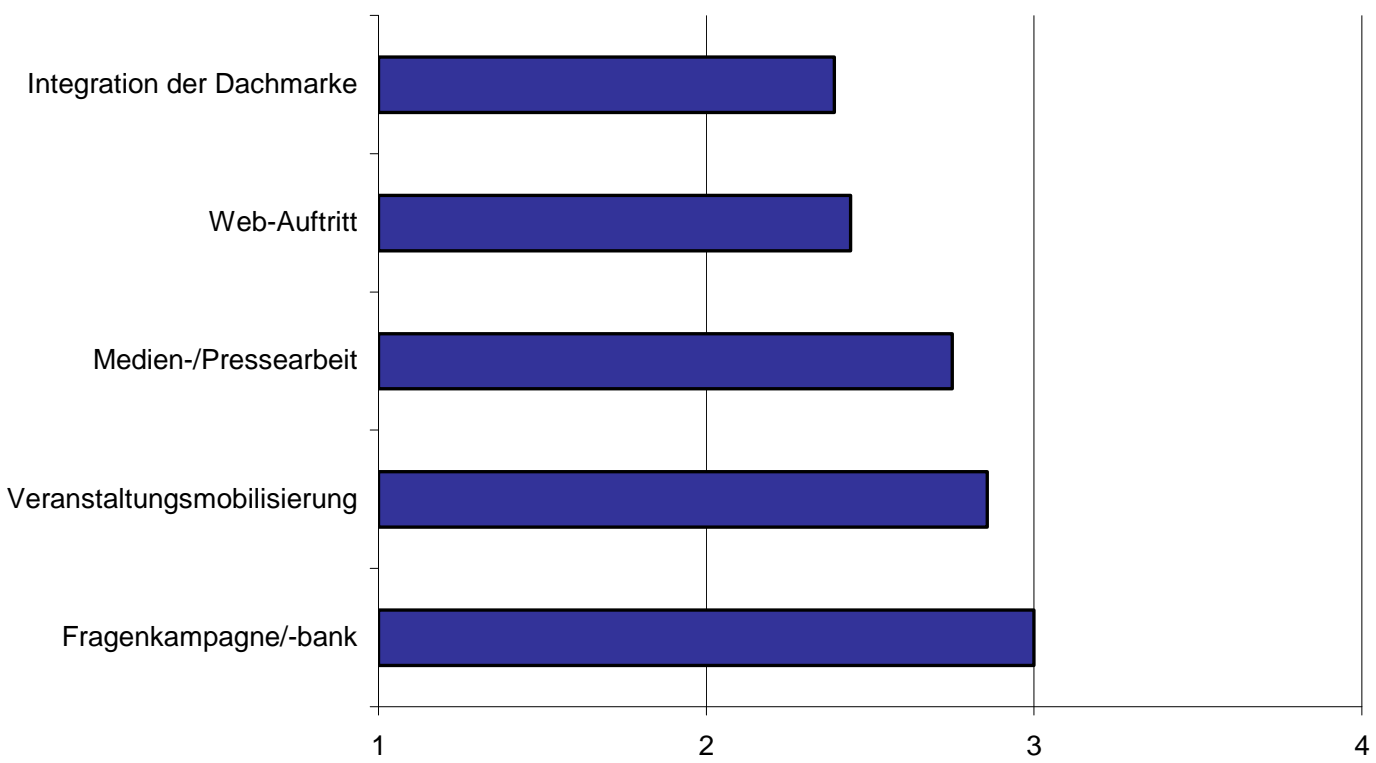

Anmerkungen:

$\mathrm{N}=21-24$

Bewertung infolge der Frage: „Wie bewerten Sie die PR-Arbeit der Kampagne?“ Mittelwerte der Bewertungen nach Schulnoten von 1 = sehr gut" bis 5 = nicht genügend

Quelle: KMU FORSCHUNG AUSTRIA, Online-Befragung Frühjahr 2006

Die eher verhaltene Bewertung der PR-Arbeit im Rahmen der Kampagne innovativesoesterreich.at kontrastiert mit der Bedeutung, die dieser von den Projektträger/innen für den Erfolg der Kampagne zugemessen wird (Grafik 15): PR-Arbeit und Verwendung der Dachmarke haben aus Sicht der Projektträger/innen eine hohe Bedeutung für den Kampagnenerfolg. Dies gilt auch für die Arbeit der Lead-Agenturen, deren Rolle zahlreiche Projektträger/innen gerade in der projektbezogenen Unterstützung und Organisation bei der PR- und Medienarbeit verorteten. Allerdings fällt auf, dass die beiden Lead-Agenturen von vergleichsweise wenigen Projektträger/innen als wichtige Ansprechpartner angesehen wurden (Grafik 16).

Die Koordination durch den Auftraggeber wird sowohl für den Kampagnen- als auch für den Projekterfolg als am wichtigsten eingestuft (Grafik 15). Diese Bewertung durch die Projektträger/innen ist in Verbindung mit dem Umstand zu sehen, dass sie gleichzeitig als wichtigen Ansprechpartner für die Durchführung ihrer Projekte vorzugsweise den Rat für FTE nennen (Grafik 16). Hier kommt zum Ausdruck, dass die Koordination der Kampagne innovativesoesterreich.at faktisch in sehr hohem Maße von der Geschäftsstelle des Rates für FTE wahrgenommen wurde und dass der Kampagnenerfolg erheblich dem dort entfalteten Aktivitätsniveau geschuldet ist.

Auffällig ist zudem, dass die befragten Projektträger/innen durchgehend der Ansicht sind, eine gute Koordination, PR- und Medienarbeit und Arbeit der Lead-Agenturen sowie der Informationsaustausch seien für den Erfolg der Kampagne jeweils bedeutsamer als für den Erfolg ihrer Projekte. Dieser Befund reflektiert möglicherweise die (zutreffende) Einschätzung, dass der Erfolg der komplex strukturierten Kampagne in sehr viel höherem Maße von den genannten, projektextern zu erbringenden Koordinationsleistungen abhängt als der Erfolg der Einzelprojekte, der - ganz abgesehen von der vergleichsweise weniger komplexen Steuerungsaufgabe wesentlich auch durch die Einbettung der Projektträger/innen in ihre "gewachsenen“ Kontaktstrukturen determiniert wird. 


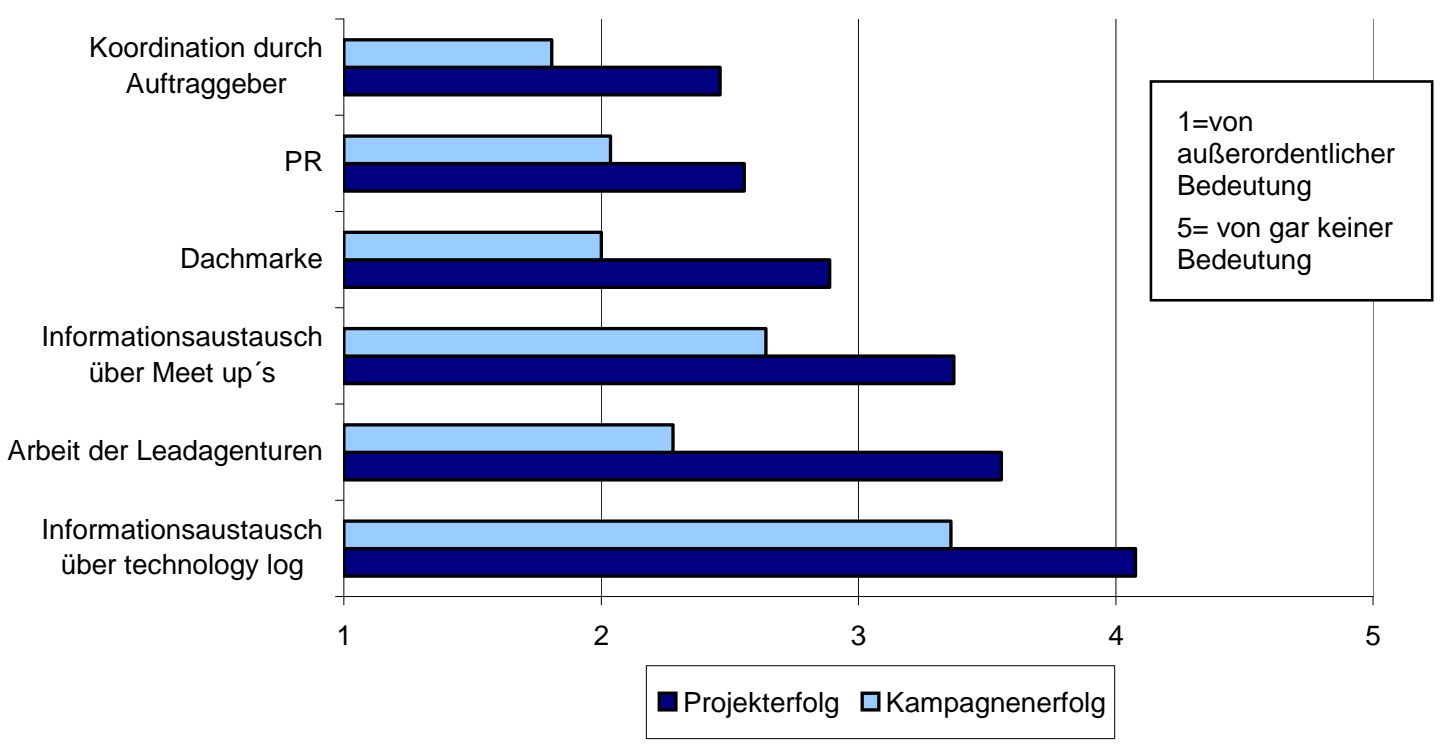

Anmerkungen:

$\mathrm{N}=24-27$

Bewertung infolge der Fragen: „Welche Bedeutung haben folgende Aspekte für den unmittelbaren Projekterfolg im Rahmen der Kampagne?" bzw. „Welche Bedeutung haben folgende Aspekte für den Erfolg der gesamten Kampagne innovatives-osterreich.at?" Mittelwerte der Bewertung von $1=$ von außerordentlicher Bedeutung bis $5=$ von gar keiner Bedeutung

Quelle: KMU FORSCHUNG AUSTRIA, Online-Befragung Frühjahr 2006

\section{Grafik 16 Wichtige Ansprechpartner für die Durchführung des Projekts/der Projekte}

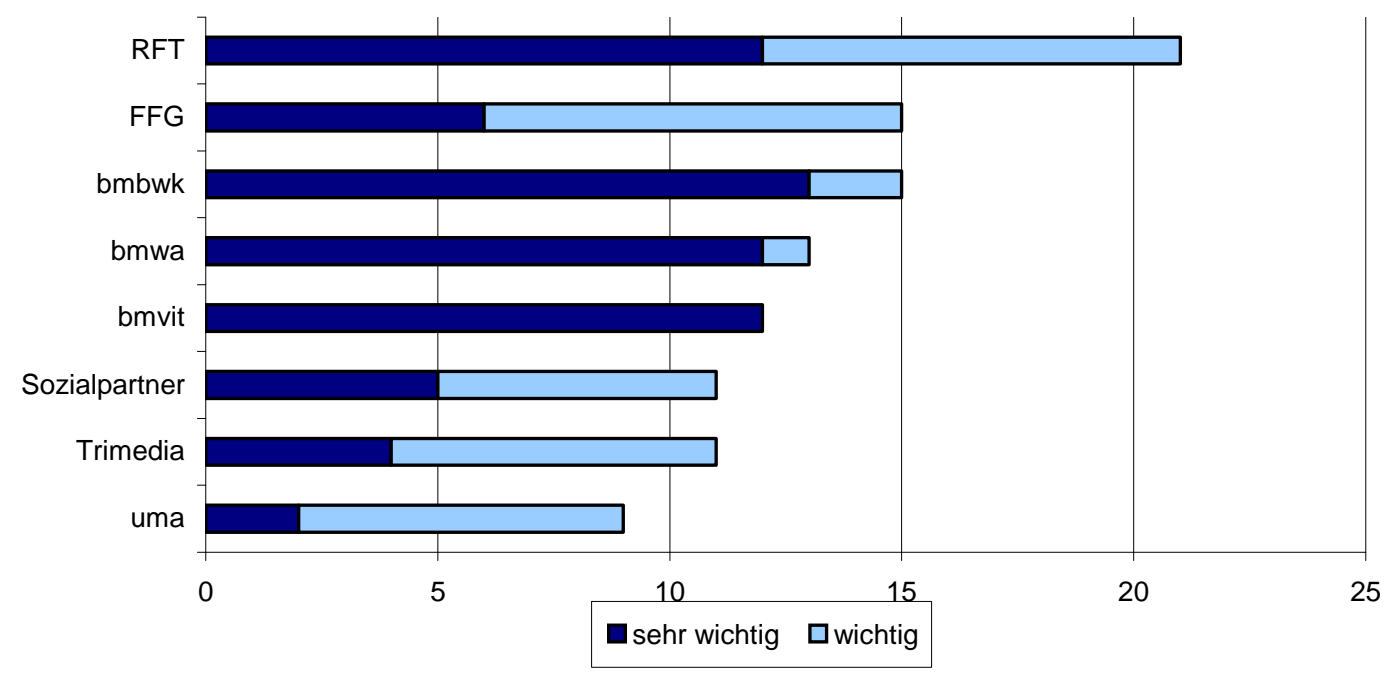

Anmerkungen:

$\mathrm{N}=21-25$

Anzahl der Antworten „sehr wichtig“ und „wichtig“ auf die Frage: „In welchem Ausmaß sind folgende Institutionen für Sie wichtige Ansprechpartner für die Durchführung Ihres Projekts/lhrer Projekte?“

Quelle: KMU FORSCHUNG AUSTRIA, Online-Befragung Frühjahr 2006 


\subsection{Community-Building?}

Ein Wunsch des Lenkungsausschusses war, die Herausbildung einer Community zwischen den Projektträger/innen zu fördern, damit diese sich besser unter dem Dach einer gemeinsamen Kampagne wiederfinden, deren Kohärenz durch so ein Community-Building unterstützt werden könnte. Die Befragung der Projektträger/innen im Rahmen dieser Evaluierung zeigt, dass tatsächlich die Hälfte der Projektträger/innen „Community-Building“ für eine „sehr wichtige“ Funktion der Dachkampagne innovatives-oesterreich.at hält; weitere $35 \%$ halten diese Funktion für „wichtig“.

Ein wesentliches Ausgangsdatum wurde dabei dadurch markiert, dass bei 20 von 27 befragten Projektträger/innen Kontakte mit anderen Projekten bzw. deren Projektträger/innen schon vor Projektbeginn bestanden; in keinem Fall wurde angegeben, dass es keine Kenntnisse über andere Projekte bzw. Projektträger/innen gäbe (Grafik 18). ${ }^{23}$ Zu berücksichtigen ist allerdings, dass die Projektträger/innen nicht nur Partner im Rahmen der Kampagne innovativesoesterreich.at sind, sondern auch Konkurrenten, was das Kooperationspotential und die Möglichkeiten des Community-Buildings naheliegenderweise einschränkt.

Um das Community-Building zu forcieren, wurden vier Formen des organisierten Informationsaustauschs eingesetzt: Die Lead-Agentur organisierte eingangs ein Kick-off Treffen und in der Folge vier Meet-up's bzw. Treffen der Projektträger/innen sowie von Vertretern des Programmkomitees, die einen informellen Informationsaustausch ermöglichen sollten. Darüber hinaus wurde die Social-Software-Plattform „technology log“ im Rahmen von innovatives-oesterreich.at eingesetzt; zu den „klassischen“ Informationswege zählte zudem der E-Mail Verkehr - insbesondere wurden die Projektträger/innen über E-Mail von laufenden Veranstaltungen im Rahmen diverser Projekte über die Lead-Agentur unterrichtet. Im Einzelnen:

Die meistgenutzten Kommunikationswege zu Trägern und Organisatoren der Kampagne sind zweifelsohne der E-Mail-Verkehr und die persönliche Kommunikation (Grafik 17). So geben sieben der Projektträger/innen an, „sehr häufig“ (mehrmals wöchentlich) und 11 „häufig“ (mehrmals pro Monat) mit Programmträgern und -organisatoren per E-Mail oder telefonisch zu kommunizieren. Den Weg des persönlichen Kontakts pflegen drei „sehr häufig“ und weitere zehn „häufig“. Meet-up's fanden zwar nur selten statt, gleichwohl gaben drei von vier der Befragten an, an diesen Veranstaltungen teilgenommen zu haben. Die Bedeutung dieser Treffen für den unmittelbaren Projekterfolg im Rahmen der Kampagne wird nach dem Schulnotensystem mit durchschnittlich 3,5 bewertet, also als eher gering eingestuft, wobei immerhin sieben der 26 Antworten mindestens die Note „2“ vergeben. Demgegenüber fällt das Ergebnis bezüglich der Bedeutung für den Erfolg der gesamten Kampagne innovatives-oesterreich.at mit der Note „2,7“ relativ positiv aus.

2317 Projektträger/innen sind über die Meet-up's über andere Projekte informiert, lediglich vier Projektträger/innen über die Plattform technology log. Neun Befragte haben aufgrund von Projektaktivitäten im Rahmen der Kampagne Kenntnis von anderen Projekten. 


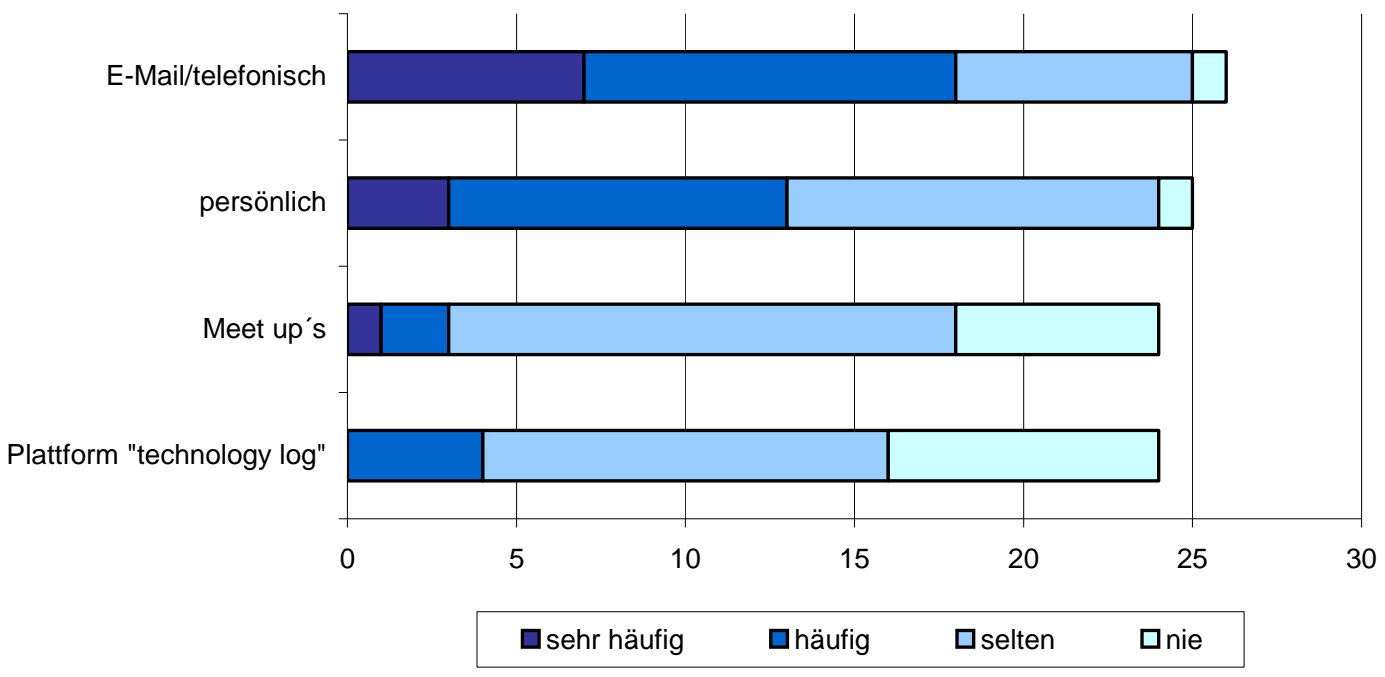

Anmerkungen:

$\mathrm{N}=24-26$

Anzahlen der Antworten auf die Frage: „Auf welchem Weg kommunizieren Sie mit Programmträgern und -organisatoren der Kampagne innovatives-oesterreich.at?"

Quelle: KMU FORSCHUNG AUSTRIA, Online-Befragung (Projektträger); Mai/Juni 2006

Die Plattform „technology log“, eine Informationsaustauschplattform auf Webbasis, wird lediglich von vier der 24 Projektträger/innen, die sich zu der Frage äußerten, „häufig“ - also mehrmals pro Monat - genutzt; eine Hälfte nutzt diese „selten“, ein Drittel gibt an, technology log „nie“ zu nutzen. Diese relativ geringe Nutzung kontrastiert mit dem für die Installation dieser Kommunikationsplattform betriebenem Aufwand. Als Erklärung für diese schwache Performance können unterschiedliche Ursachen identifiziert werden; an dieser Stelle soll nur auf jene Aspekte eingegangen werden, die mit der Struktur, dem Design, und dem Management des Programms zu tun haben.

Nach seiner ursprünglichen Konzeption sollte technology log die gesamte Forschungsförderungs- und Innovationsszene einbinden und „Innovation in das Kommunikationsportfolio hineinbringen." ${ }^{\text {"24 }}$ Das Projekt startete mit einer Analyse 2004 und einem ersten Roll-Out Anfang 2005. Daraufhin folgte eine Neuorientierung, mit Fokus auf die Initiative innovatives-oesterreich.at, der das Tool zur Verfügung gestellt werden sollte. Der Zeitplan war jedoch ungünstig: Einerseits bestand das (Ressort-)Projekt schon vor der Ausschreibung der Kampagne, andererseits war damals die Neuorientierung noch nicht fixiert. Weder im Ausschreibungstext noch in den Verträgen wird deshalb konkret auf technology log als die Kommunikationsplattform hingewiesen, eine Nutzung ist nicht bindend vorgesehen, sondern erfolgt auf freiwilliger Basis. Insbesondere sind auch die Lead-Agenturen nicht zur Kooperation - also zum Datenaustausch - verpflichtet. Es entstehen Doppelgleisigkeiten, erstens was die bereitgestellte Information auf der Homepage der Kampagne und der Plattform technology log betrifft, zweitens in der Kommunikation, da Informationen nicht nur auf die Plattform gestellt werden, sondern parallel auch per E-Mail versandt werden. Da die Plattform keine aktive Informationspolitik betreibt, sondern einen Raum bietet, um Informationen bereitzustellen und selektiv abzurufen, „zieht sie den Kürzeren“. Der Versuch, in Rahmen eines Meet-up's alle Projektträger/innen einzuschulen und zur Verwendung des Tools zu bewegen, ist nur teilweise erfolgreich, das Vertrauen, hier alle wesentlichen Informationen zu erhalten, und durch die Bereitstellung von Information auch alle wesentlichen Zielgruppen zu erreichen, wird nicht aufgebaut.

24 Interview mit dem Projektleiter. 
Für das Community-Building ist weiterhin von Bedeutung, auf welchen Wegen die Akteure und hier insbesondere die Projektträger/innen - voneinander Kenntnis erhalten. Die Befragung zeigt, dass dem Großteil der Projektträger andere Projekte durch unterschiedliche Quellen bekannt wurden. Ebenso wie durch das Kick-off Meeting kannten 20 der Befragten andere Projektträger schon vor Projektbeginn, unabhängig davon nennen 17 von 27 die Meet-up's als Quelle für die Kenntnis anderer Projektträger. Über Projektaktivitäten im Rahmen der Kampagne kennt ein Drittel der Befragten andere Projektträger/innen im Rahmen der Kampagne. Bezeichnenderweise informieren sich nur vier von 27 mit Hilfe des technology log über andere Projekte bzw. Projektträger/innen (Grafik 18).

Grafik 18 Gründe der Kenntnis anderer Projekte bzw. Projektträger/innen der Kampagne

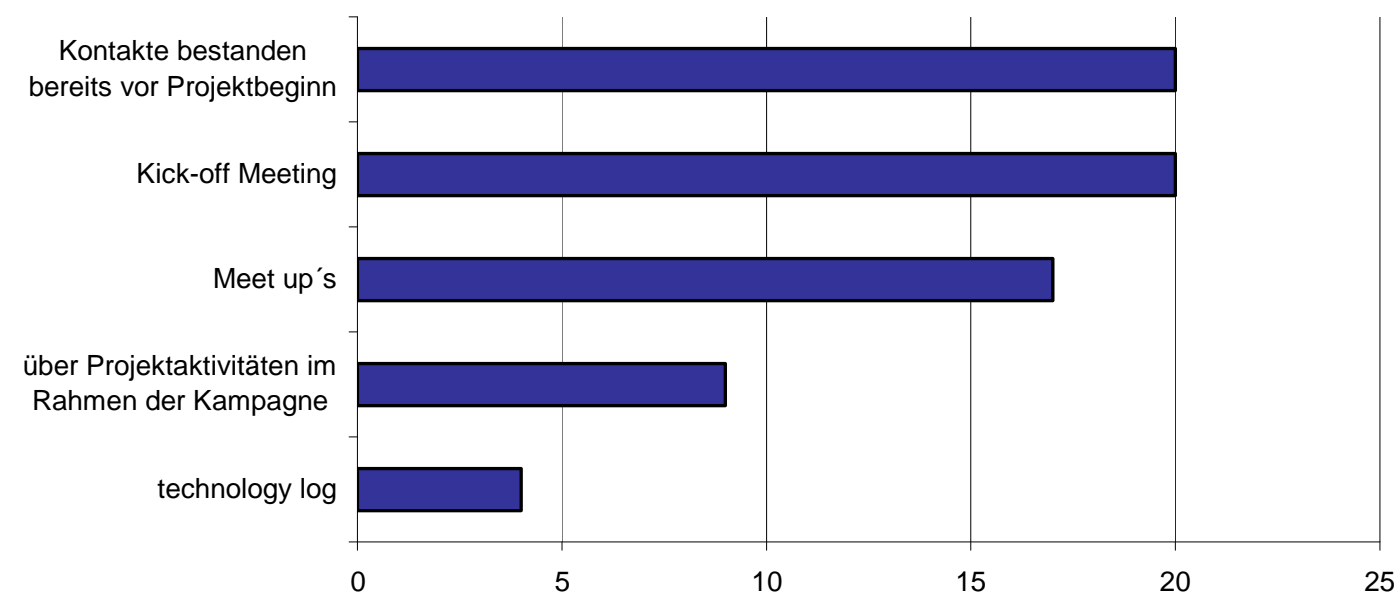

Anmerkungen:

$\mathrm{N}=27$, Mehrfachantworten möglich

Anzahl der Nennungen auf die Frage: „Woher kennen Sie andere Projekte bzw. deren Projektträger der Kampagne innovatives-oesterreich.at?"

Quelle: KMU FORSCHUNG AUSTRIA, Online-Befragung (Projektträger); Mai/Juni 2006

\subsection{Organisatorisches Lernen}

Eine Besonderheit der Kampagne innovatives-oesterreich.at ist das dargestellte organisatorische Setting (vgl. Kapitel 3.1). Die Tatsache, dass es im Zuge der Kampagne zu einer "Steuerungskrise“ kam, die auch die Organisationsstruktur betraf, könnte zu dem Befund führen, diese als schwach zu beurteilen. Gegen eine solche Einschätzung spricht aber, dass ein deutliches organisatorisches Lernen zu beobachten war, das die Grundlagen für eine Stabilisierung weniger Kernelemente bildet.

Im Folgenden wird diese Entwicklung entlang der verschiedenen Positionen der beteiligten Akteure, die aus Gesprächen mit Akteuren der Programmsteuerung identifiziert werden konnten, dargestellt. Weiters wird die Dynamik der Anpassung in dem gegebenen Setting einer interministeriellen und vom Rat für FTE koordinierten Plattform skizziert. Schließlich werden jene Punkte zusammengefasst, die als organisatorisches Lernen für kommende Kampagnen genützt werden können.

Die Positionierung der Initiative innovatives-oesterreich.at im öffentlichen Raum war im Beobachtungszeitraum umstritten, insbesondere hinsichtlich ihrer Sichtbarkeit und Wirksamkeit einerseits für eine breitere Öffentlichkeit, andererseits für spezifische Zielgruppen. Im Hintergrund dieser Diskussion stand der Wechsel in der Konzeption von einer „klassischen“ Werbekampagne in der ersten Kampagnenphase hin zu interaktiven und zielgruppenspezifischen Vermittlungsprojekten in der zweiten Kampagnenphase. Die Abwendung von "traditionellen“ 
Werbemaßnahmen erfolgte seinerzeit auch auf Basis der Evaluierungsergebnisse, die zwar die Sichtbarkeit der Marke bestätigten, jedoch in Frage stellten, inwiefern die Marke bzw. Werbung auch den erwünschten Inhalten zugeordnet wurde (Schweiger et al. 2003, vgl. Fußnote 11 in Kap.2.2).

Die „Sichtbarkeit“ einer Kampagne zur Sensibilisierung für Wissenschaft, Forschung und Technologie betrifft unterschiedliche Ebenen, und zwar Sichtbarkeit

- der Marke und Dachkampagne www.innovatives-oesterreich.at,

- der Projekte,

- des Nutzens von Wissenschaft, Forschung und Technologie,

- und nicht zuletzt die der einzelnen Akteure.

Optimalerweise trägt die Sichtbarkeit der einen, im Rahmen einer solchen Kampagne praktizierten Aktivität zur jener der anderen bei: Je präsenter die Marke, desto leichter ist es, Aufmerksamkeit für Projekte zu schaffen, und umgekehrt, erfolgreiche Projekte erleichtern die Kommunikation einer Dachmarke.

Die Sichtbarkeit jeglicher Akteure ist nicht Ziel der Kampagne, jedoch zeigte sich im Hintergrund, dass dies ein sensibler Punkt ist, jedenfalls was das Anbringen von Logos der beteiligten Akteure betrifft. Hier ist wiederum zwischen den Programmträgern - Ministerien und Rat für FTE - den Projektträgern, und der Plattform zu unterscheiden. Der Zeitplan der Projekte hat hier zu Spannungen, und damit zu einem Managementaufwand geführt, da das größte Projekt der Kampagne, die Lange Nacht der Forschung 2005 (Teil der X.PERIMENTA), so knapp nach Verabschiedung des Kommunikationskonzepts der Lead-Agenturen startete, dass eine Abstimmung kaum mehr möglich war. So bewegte sich die X.PERIMENTA - die der Projektgröße entsprechend eine eigene Öffentlichkeitsarbeit projektiert hatte - auf einem vom Verlauf der restlichen Kampagne unabhängigen Pfad. Der Projektträger musste immer wieder angehalten werden, das Logo der Dachkampagne in sein Kommunikationsmaterial zu integrieren, und tat dies teilweise nur halbherzig, im Wortsinne in Grautönen.

Dementsprechend wurden Ende 2005 erste Stimmen laut, die darauf hinwiesen, dass die Präsenz der Dachmarke unzureichend ist, und die Koordination der Kampagne zu wünschen übrig lässt. Gleichzeitig wurde der mit dem Wechsel des Konzepts verbundene Verlust an Sichtbarkeit (Plakate, Fernsehspots, Radio) von Anhängern „klassischer“ Werbemaßnahmen, insbesondere vom Vorsitzenden des Rates für FTE, nicht goutiert. Das Problem der Sichtbarkeit stand damit auf der Tagesordnung. Diese Kritik, die letztlich auch die inhaltliche Konzeption der Kampagne tangierte, führte in Verbindung mit dem geplanten Aufbau des Managements für die zweite Kampagnenphase sowie nicht geplanten oder planbaren Gegebenheiten, die Anpassungen notwendig machten, im Zeitraum um den Jahreswechsel 2005/2006 zu sich wechselseitig verstärkenden Spannungen:

- Das Abrücken von „klassischen“ Werbemaßnahmen wurde insbesondere vom Vorsitzenden des Rates für FTE als Verlust empfunden, da es keine breite öffentliche Präsenz der Kampagne mehr gab.

- Der Aufwand für die Koordination der Kampagne nahm mit fast monatlichen Sitzungen des Programmkomitees und der weiteren begleitenden Gruppen überhand.

- Man musste einsehen, dass das Zusammenführen zweier Agenturen, die bisher keine Kooperationserfahrung hatten, aufwendig war, und die Erledigung einiger wesentlicher Steuerungsaufgaben unerledigt blieben. Dies betraf Punkte wie eine zufrieden stellende und vollständige Formulierung der Pflichtenhefte für die jeweiligen Akteure, Funktionen eines Sekretariats des Programmmanagements, die teilweise an der zuständigen Person in der Geschäftsstelle des Rates für FTE „hängen blieben“, aber auch Maßnahmen der Öffentlichkeitsarbeit.

- Bezüglich des letzten Punktes entstand im Zuge der Diskussionen ein Konsens, dass die beiden gewählten Agenturen die Vermittlung von Forschung- und Technologie nicht zum Kerngeschäft haben, und nur unzureichend in eine "Community“ integriert sind, die an sich 
recht übersichtlich ist, und dementsprechend auch über persönliche Vernetzung und informellen Informationsaustausch funktioniert.

- Es galt also eine Lösung zu finden, die die Sichtbarkeit der Marke erhöht, die von einer Person oder Firma getragen wird, die einerseits Zugang zu der genannten Community hat, und andererseits Kompetenz in Projektmanagement und Koordination aufweist.

Die Folge dieser "Steuerungskrise“ - in deren Verlauf auch ein Abbruch der Kampagne, wenn auch nicht sehr realistisch, vorgeschlagen wurde - waren ein als durchaus "wertvoll“ zu bezeichnender Lernprozess und Anpassungen im Programm. Zunächst erschien die Lösung des Problems als Quadratur des Kreises, jedenfalls solange von einem Programmmanager die Rede war, solange also die Aufgabe recht hoch angesiedelt war. Schließlich gelang es, einen Koordintor für begleitende Maßnahmen zu identifizieren, der in Kooperation mit den LeadAgenturen für die verbleibende Zeit - die Halbzeit der Kampagne war bereits abgelaufen - die weiteren Schritte der Öffentlichkeitsarbeit konzipierte. Für diese Aktivitäten wurde ein Teil des von den Lead-Agenturen verwalteten Budgets zweckgewidmet.

Damit war ein entscheidender Schritt zur konstruktiven Kooperation getan: Was schriftliche Vereinbarungen nicht leisten konnten, war durch den zeitlichen Zusammenfall der Bestellung eines unabhängigen Dritten und des geeigneten Zeitpunkts, nach ausreichend langem gegenseitigen Kennenlernen sowie nach einer überstandenen Krise, möglich. Dadurch gelang es auch, neben einer verbesserten Öffentlichkeitsarbeit, die ja Gegenstand der begleitenden Maßnahmen war, die Koordination zwischen den Lead-Agenturen zu verbessern und die zahlreichen programmbegleitenden Komitees zusammenzuziehen. Statt getrennter Sitzungen von Programmkomitee, Lenkungsausschuss und Steuerungsgruppe (vgl. Kapitel 3.2) wurden nunmehr Lenkungsausschuss und Steuerungsgruppe zusammengezogen; diese trafen sich im Anschluss an die Sitzung des Programmkomitees - das heißt, nach einem ersten Sitzungsteil, an dem nur Ministerien und Rat für FTE teilnahmen, wurden sowohl Lead-Agenturen als auch Science Communications, die für die X.PERIMENTA zuständige Agentur, hinzugezogen.

Jenseits dieses Lernprozesses im Rahmen der zweiten Kampagnenphase fällt auch auf, dass es zwischen erster und zweiter Kampagne zu einer Ausdifferenzierung und Professionalisierung im Programmmanagement kam: Erstens weil neben Ressort- und Poolprojekten die Ausschreibung der Kampagne in Modulen im Zentrum der zweiten Phase stand. Zweitens wurden erste wesentliche Schritte der Ausgliederung von Koordinationsfunktionen an spezialisierte Agenturen, teils öffentlich und teils privat, vollzogen. Auch fällt auf, dass die Sozialpartner in der zweiten Phase nicht mehr in die Kampagnenkonzeption eingebunden waren, Politik wurde also teilweise durch Professionalisierung abgelöst. Gerade diese Ausdifferenzierung deckte jedoch auf, dass die Schnittstellen zwischen den unterschiedlichen Akteuren nur unzureichend definiert waren. Da Versäumnisse bei der Definition von Aufgaben im Rahmen von Auslagerung nur äußerst begrenzt nachzuholen sind, haben diese Probleme die Organisation der Kampagne über eine weite Strecke begleitet.

Tabelle 4 fasst Entwicklungen in der Organisation von der ersten zur zweiten Kampagne zusammen und stellt diesen Strukturen jene Aspekte gegenüber, die im Zuge der zweiten Kampagne als „organisationales Lernen“ bezeichnet werden können - und die nach unseren Beobachtungen weitgehend unbestritten sind. Thematisiert werden hier die Aspekte der Zusammensetzung und Aufgaben des Programmkomitees, die Vergabemodalitäten der Projekte, die Rolle von Agenturen - sowohl die Lead-Agenturen als auch die FFG betreffend -, die Positionierung von Pool- oder Kampagnenprojekten, sowie der Zugang zur Sichtbarkeit der Kampagne. 


\begin{tabular}{|c|c|c|}
\hline Erste Kampagne & Zweite Kampagne & Gelerntes \\
\hline $\begin{array}{l}\text { Programmkomitee, zuständig für } \\
\text { Konzeption und Begleitung. }\end{array}$ & $\begin{array}{l}\text { Programmkomitee, zuständig für Konzeption und } \\
\text { Begleitung. }\end{array}$ & $\begin{array}{l}\text { In der Bewältigung der Begleitung ist das Zusammenziehen aller } \\
\text { beteiligten Akteure sinnvoll. }\end{array}$ \\
\hline $\begin{array}{l}\text { Der Vorsitzende des Rates für } \\
\text { FTE hat die Aufgabe des } \\
\text { Vorsitzes des Programmkomitees } \\
\text { weitestgehend wahrgenommen. }\end{array}$ & $\begin{array}{l}\text { Der Vorsitzende des Rates für FTE hat sich in } \\
\text { seiner Funktion als Vorsitzender des } \\
\text { Programmkomitees bis auf die erste Sitzung } \\
\text { durch den Leiter der Geschäftsstelle vertreten } \\
\text { lassen, später durch den Sachbearbeiter in der } \\
\text { Geschäftsstelle. }\end{array}$ & $\begin{array}{l}\text { Die Geschäftsstelle des Rates für FTE ist personell nicht als Sekretariat } \\
\text { eines Ressort-übergreifenden Programms ausgestattet. } \\
\text { Programmatische Entscheidungen und operative Begleitung können } \\
\text { getrennt werden, und unterschiedliche Akteure einbinden. }\end{array}$ \\
\hline $\begin{array}{l}\text { Keine Ausschreibung von } \\
\text { Projekten }\end{array}$ & $\begin{array}{l}\text { Ausschreibung von Projekten im Rahmen von } \\
\text { Modulen }\end{array}$ & $\begin{array}{l}\text { Die Ausschreibung der Projekte im Rahmen von wurde sehr positiv } \\
\text { angenommen. }\end{array}$ \\
\hline $\begin{array}{l}\text { Keine Ausschreibung der } \\
\text { Funktion einer Lead-Agentur, } \\
\text { sondern freie Projektvergabe }\end{array}$ & $\begin{array}{l}\text { Ausschreibung der Aufgaben einer Lead-Agentur } \\
\text { und eines Internetauftritts als zwei Module der } \\
\text { gesamten Ausschreibung der Kampagne }\end{array}$ & $\begin{array}{l}\text { Die Ausschreibung der Aufgaben einer Lead-Agentur soll der } \\
\text { Ausschreibung der Projekte vorgelagert sein. }\end{array}$ \\
\hline $\begin{array}{l}\text { Freie Vergabe an eine Agentur } \\
\text { für eine Werbekampagne }\end{array}$ & Vergabe der Lead-Funktionen an zwei Agenturen & $\begin{array}{l}\text { Die Vergabe der Dachfunktionen an zwei verschiedene Agenturen war } \\
\text { eine von einer Pattsituation geprägte Lösung, die unbeachtet der } \\
\text { Qualitäten und Schwächen der beiden Agenturen als problematisch } \\
\text { erkannt wurde. Die Schwäche lag vor allem im Ausschreibungstext, der an } \\
\text { sich eine Errungenschaft war, aber eben auch eine erste Runde, aus der } \\
\text { gelernt werden kann. In Zukunft gilt es } \\
\text { - } \quad \text { Inhaltliche Arbeit und Projekt- und Programmmanagement nicht zu } \\
\text { vermischen. Insbesondere soll die Lead-Agentur nicht auch } \\
\text { Konkurrent der anderen Projektträger/innen sein. } \\
\text { Projektmanagement muss an sich nicht innovativ sein. } \\
\text { - } \quad \text { nur eine einzige Lead-Agentur zu bestellen. } \\
\text { - } \quad \text { die Aufgaben der Lead-Agentur als Dienstleistungen für die } \\
\text { Gesamtkampagne und für die Projektträger zu konzipieren }\end{array}$ \\
\hline Keine Ausschreibung & Durchführung der Ausschreibung durch die FFG & $\begin{array}{l}\text { Die Durchführung durch die FFG ist erfolgreich, jedoch ist es notwendig, } \\
\text { die Schnittstellen zwischen Lead-Agentur und FFG, insbesondere } \\
\text { bezüglich der Berichtlegung, im Vorhinein zu definieren. Weiters kann die } \\
\text { FFG, so die Rollen klar definiert sind, in die operative Begleitung verstärkt } \\
\text { involviert werden. }\end{array}$ \\
\hline
\end{tabular}




\begin{tabular}{|c|c|c|}
\hline Erste Kampagne & Zweite Kampagne & Gelerntes \\
\hline $\begin{array}{l}\text { Informelles Community } \\
\text { Bildung }\end{array}$ & $\begin{array}{l}\text { Unterstützung der Community Bildung durch eine } \\
\text { webbasierte Informationsaustauschplattform } \\
\text { (technology log), durch ein Kick-off Meeting und } \\
\text { durch Meet-up's }\end{array}$ & $\begin{array}{l}\text { Der Austausch in der Community stößt teilweise durch die Konkurrenz der } \\
\text { Projektträger untereinander an Grenzen. Die Plattform technology log hatte } \\
\text { unter gegebenen Umständen, die eine Vernetzung mit der Homepage } \\
\text { www.innovatives-oesterreich.at unterbanden, und durch unklare } \\
\text { Programmmanagementverhältnisse keine wahre Chance, ihr Potential } \\
\text { auszuschöpfen. Auch war ihre „Anwendung“ in der Kampagne kein Resultat } \\
\text { der vorangehenden Feasibility-Untersuchungen, die Bedarfsanalyse war } \\
\text { unvollständig. Schließlich ist die Trennung der Moderation sozialer } \\
\text { elektronischer Kommunikation und sonstiger Kommunikation nicht } \\
\text { zielführend. } \\
\text { Prinzipiell wird die Community-Bildung als hilfreich für die Kampagne } \\
\text { eingeschätzt }\end{array}$ \\
\hline Poolprojekte & Poolprojekte und ausgeschriebene Kampagne & $\begin{array}{l}\text { Die Plattform garantiert den Projektträgern unter anderem einen } \\
\text { unabhängigen Kunden; nicht Ressortinteressen sondern allein die } \\
\text { Dachmarke ist bei Vermittlungsaktivitäten zu berücksichtigen. }\end{array}$ \\
\hline $\begin{array}{l}\text { Sichtbarkeit durch } \\
\text { gemeinsames Logo, } \\
\text { Homepage, } \\
\text { Medienkooperationen und } \\
\text { klassische Werbung }\end{array}$ & $\begin{array}{l}\text { Sichtbarkeit durch gemeinsames Logo, } \\
\text { Homepage, Medienkooperationen, ohne } \\
\text { klassische Werbung. } \\
\text { Krise der „Sichtbarkeit“, Bestellung eines } \\
\text { begleitenden Koordinators für Medienarbeit, } \\
\text { punktuelle Projektübergreifende medial wirksame } \\
\text { Maßnahmen, Schaltungen im Infoscreen der } \\
\text { Wiener U-Bahnen }\end{array}$ & $\begin{array}{l}\text { Der Abgang von klassischer Werbung wird von allen drei Ressorts, weniger } \\
\text { vom Rat für FTE, getragen. } \\
\text { Im Zuge der Kampagne kristallisierte sich die Meinung im Programmkomitee } \\
\text { heraus, dass erfolgreiche Präsenz der Kampagne im öffentlichen Raum nur } \\
\text { von einer Person gesichert sein, die bestens anschlussfähig ist, sowohl für } \\
\text { Medienarbeit als auch für Wissenschaftskommunikation, die also die } \\
\text { Spezifika gerade der Awareness-Bildung kennt. Reinen Marketingfirmen wird } \\
\text { diese Kompetenz allgemein nicht zugetraut. } \\
\text { Beim Design einer eventuellen dritten Kampagne sollte auf diese Profil- } \\
\text { Anforderung ein besonderes Augenmerk gerichtet werden. }\end{array}$ \\
\hline
\end{tabular}


Auch wenn sich die beteiligten Akteure mit diversen Problemen auseinandersetzen mussten, so scheinen die genannten Lerneffekte für eine kommende Runde gute Voraussetzungen zu bieten, um effizientere Strukturen zu finden. Aus Sicht der Evaluation wären dabei folgende Punkte zu berücksichtigen:

- Beibehaltung einer ressortübergreifenden Plattform innovatives-oesterreich.at.

- Klar definierte Aufgaben für eine Lead-Agentur, jedoch getrennte Ausschreibung dieser Funktion.

- Durchführung der Ausschreibung der Kampagne sowie der operativen Projektbetreuung durch die FFG. Wie oben erwähnt, war die gemeinsame Ausschreibung der Projekte für die Kampagne sowohl im Sinne der Transparenz als auch der inhaltlichen Konsistenz der Projekte ein Gewinn. Ihre Abwicklung durch die FFG verlief gut, auch ist die FFG im Unterschied beispielsweise zu privaten Agenturen - groß genug, um die zeitgerechte Abrechnung der Projekte zu garantieren.

- Begleitung der Kampagne durch ein gemischt besetztes Komitee, jedoch funktionale Trennung von Konzeption und Programmdesign einerseits und Programmbegleitung andererseits. Diese garantiert im Wesentlichen die Einhaltung der Vorstellung von jenem.

- Explizite Aufzählung der Anforderungsprofile und Anforderungen in den Ausschreibungsunterlagen:

- Klare Positionierung hinsichtlich der Mindestanforderungen an die Präsenz im öffentlichen Raum: Projekte und/oder Dachmarke, Hierarchie dieser Aspekte, Zuordnung der Budget (Dachbudget oder spezifisches Budget für jedes Projekt, aber von der Lead-Agentur verwaltet?).

- Anforderungen an Dachfunktionen: Webpage, Programmmanagement, Community-Building, Pressearbeit, sonstige PR, Coverage der Projekte (nur gemeinsam finanzierte Projekte oder auch Ressortprojekte oder auch Informationsleistung über sonstig finanzierte Projekte in Österreich...).

- Anforderungen an die Projektleiterinnen bezüglich Informationsaufbereitung, Berichtlegung.

- Zeitmanagement: Durch allgemein gültige bequeme Vorlaufzeiten kommt es zu Projekthäufungen etwa ein Jahr nach der Projektvergabe. Geeignete Maßnahmen, über gegenseitige Abstimmung oder zeitliche Platzierungen (im Rahmen des technisch möglichen) durch ein Programmmanagement könnten zu einem ausgeglichenen Zeitplan führen.

- Definition der Zielgruppe für PR-Dienstleistungen: Sollen nur kleine Projekte von einer Lead-Agentur in ihrer PR Arbeit unterstützt werden, oder soll es eine zentrale PR-Koordination geben, die auch Großprojekte einbindet? Umgekehrt sollten schon in den Ausschreibungsunterlagen die Mindestanforderungen an die Integration der Projekte in eine gemeinsame Kampagne definiert werden, wie beispielsweise die durchgehende Verwendung des gemeinsamen Logos oder die Bereitstellung von Projektunterlagen an die Lead-Agentur für eine koordinierte Pressearbeit. Es war im Zuge der zweiten Kampagne zu beobachten, dass in der Öffentlichkeit die Lange Nacht der Forschung nicht der Kampagne innovatives-oesterreich.at zugeordnet wurde. 
- Ergebnisorientierte Staffelung der Ausschreibungen und Projektvergabe: Beginnend mit der Einberufung des Programmkomitees und Definition der Zielsetzungen und Aspekte der neuen Kampagne, dann Beauftragung der FFG mit der Ausschreibung dieser Kampagne, dann Ausschreibung der Lead-Agentur, dann Ausschreibung der gesamten Kampagne, inklusive X.PERIMENTA. 


\section{$5 \quad$ Wirkungen der Kampagne}

Die Frage, ob die mit der Kampagne innovatives-oesterreich.at intendierten Ziele erreicht wurden, lässt sich letztlich nur anhand der Wirkung der Kampagne, ihrer Reichweite und Resonanz, ermessen. Es ist daher insbesondere von Bedeutung, inwieweit die Kampagne die beiden Zielgruppen „Jugendliche“ (5.1) sowie „Klein- und Mittelunternehmen“ (5.2) erreicht hat. In Form eines Exkurses wird die Wirkung der Kampagne innovatives-oesterreich.at im Vergleich zu ähnlichen Sensibilisierungskampagnen thematisiert. Vorgestellt werden im Weiteren die Ergebnisse der Wirkungsanalyse der in mehrfacher, nicht zuletzt in budgetärer Hinsicht als „Vorzeigeprojekt" zu bezeichnenden Langen Nacht der Forschung 2005 (5.3). Abschließend werden Hinweise zur Wirkung der unter dem Dach von innovatives-oesterreich.at durchgeführten Einzelprojekte gegeben (5.4).

\subsection{Zielgruppe Jugendliche}

Die in zwei Wellen - Anfang und Ende 2006 - durchgeführte repräsentative Befragung von Jugendlichen zeigt eine beachtlich hohe gestützte Bekanntheit der Kampagne innovativesoesterreich.at: Im ersten Quartal 2006 war die Kampagne jedem Vierten österreichischen Jugendlichen bekannt, im letzten Quartal 2006 sogar fast jedem Dritten (Grafik 19). Auffallend ist bei einer näheren Betrachtung, dass es differenziert nach Geschlecht, Bildungsstand und Größe des Wohnortes nur graduelle Abweichungen gibt: Bei weiblichen Jugendlichen und höher gebildeten Jugendlichen liegt die gestützte Bekanntheit etwas über dem Durchschnitt; bei Jugendlichen im städtischen Umfeld, die Anfang 2006 die Kampagne noch am wenigsten kannten, ist im letzten Quartal 2006 eine erhebliche Zunahme der Bekanntheit (um +20 \%) festzustellen.

Grafik 19 Jugendliche: Gestützte Bekanntheit ${ }^{1}$ der Kampagne innovatives-oesterreich.at (1. Quartal 2006 / 4. Quartal 2006

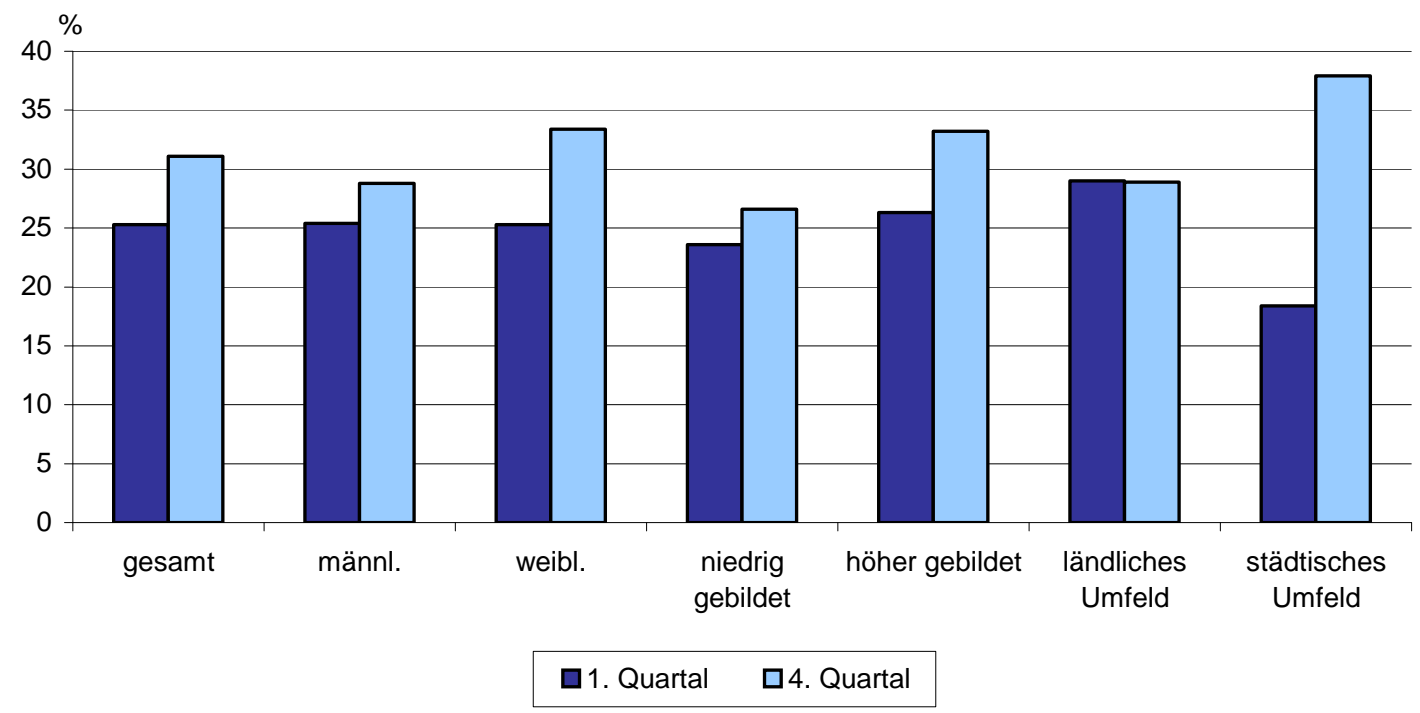

\section{Anmerkungen:}

$\mathrm{N} 1=400, \mathrm{~N} 4=403$, Grundgesamtheit: Jugendliche 14-25 Jahre in Österreich (bevölkerungsrepräsentativ gewichtet)

$\left.{ }^{1}\right)$ Prozentanteil der Ja-Antworten bezogen auf: „Wir haben nun eine Reihe von Projekten, Veranstaltungen und Initiativen aus dem Bereich Forschung, Innovation und Wissenschaft aufgelistet. Bitte kreuzen Sie alle an, die Sie zumindest dem Namen nach kennen?"

$\left.{ }^{2}\right)$ „höher gebildete Jugendliche“: Mindestabschluss einer Fach/Berufsschule

$\left.{ }^{3}\right)$ „ländliches Umfeld“: Ortsgröße unter 5.000 Einwohner," städtisches Umfeld“: Ortsgröße 100.000 und mehr Einwohner Quelle: KMU FORSCHUNG AUSTRIA, TNS Infratest, Jugendlichen-Befragung 1. Quartal 2006; 4. Quartal 2006 
Auch hinsichtlich der gestützten Bekanntheit des Logos (Ticket) der Kampagne innovativesoesterreich.at zeigt sich eine Steigerung der Bekanntheitswerte, von $17 \%$ im erstem auf $21 \%$ im letzten Quartal 2006 (Grafik 20). Im letztgenannten Zeitraum war das Kampagnen-Logo bei männlichen Jugendlichen und höher gebildeten Jugendlichen leicht, bei Jugendlichem aus dem städtischen Umfeld deutlich überdurchschnittlich häufig bekannt.

Grafik 20 Jugendliche: Gestützte Bekanntheit ${ }^{1}$ des Kampagnen-Logos (Ticket); 1. Quartal 2006 / 4. Quartal 2006

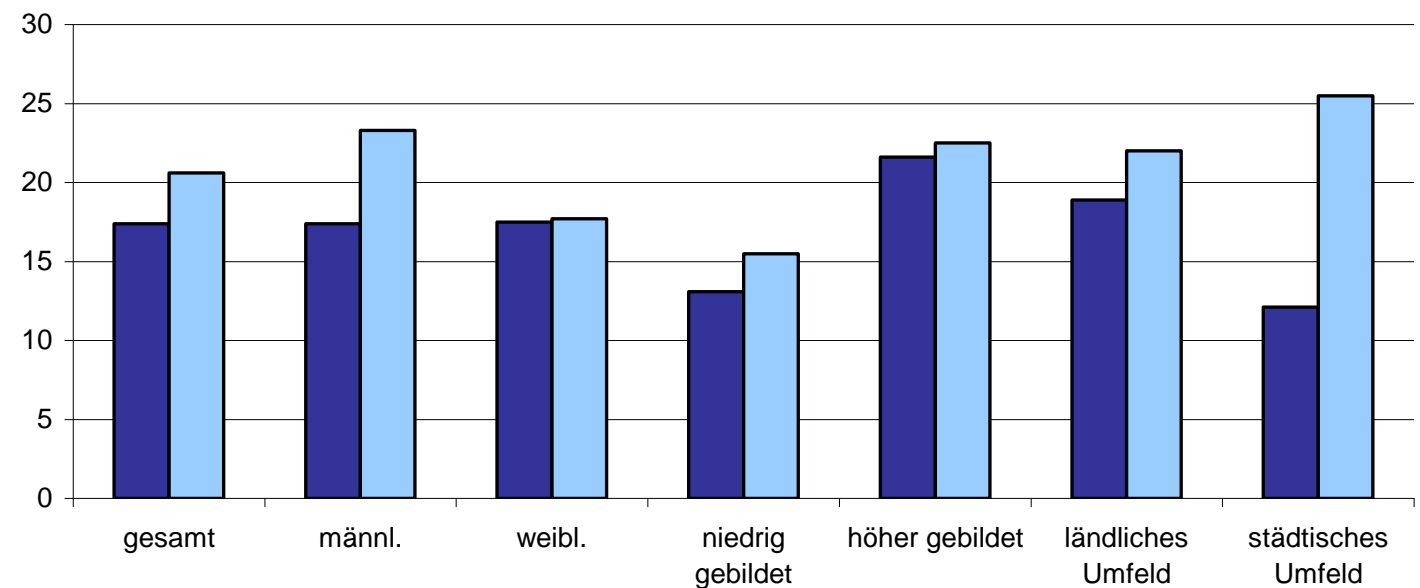

$$
\text { ㅁ. Quartal } \square 4 \text {. Quartal }
$$

Anmerkungen:

N1=400, N4=403, Grundgesamtheit: Jugendliche 14-25 Jahre in Österreich (bevölkerungsrepräsentativ gewichtet)

$\left.{ }^{1}\right)$ Prozentanteil der Ja-Antworten bezogen auf: „Bitte kreuzen Sie nun an, ob Sie das folgende Logo schon einmal in den Medien oder an anderer Stelle gesehen haben?"

$\left.{ }^{2}\right)$ „höher gebildete Jugendliche“: Mindestabschluss einer Fach/Berufsschule

$\left.{ }^{3}\right)$ „ländliches Umfeld“: Ortsgröße unter 5.000 Einwohner," städtisches Umfeld“: Ortsgröße 100.000 und mehr Einwohner Quelle: KMU FORSCHUNG AUSTRIA, TNS Infratest, Jugendlichen-Befragung 1. Quartal 2006; 4. Quartal 2006

Während die Schwankungsbreite der Bekanntheit von Kampagne und Logo hinsichtlich des Bildungsstandes der befragten Jugendlichen eher im erwarteten Bereich liegt, ist der zwischen Anfang und Ende 2006 zu beobachtende deutliche Zuwachs der Bekanntheit bei den Jugendlichen im städtischen Umfeld interpretationsbedürftig. Im Sinne einer Arbeitshypothese ist vielleicht zu vermuten ist, dass es im ersten Quartal Ausstrahlungseffekte des "Großereignisses" Lange Nacht der Forschung in den jeweils die Veranstaltungsorte umgebenden ländlichen Raum gegeben hat, die dann bei den anderen, zwar auch vornehmlich in städtisch geprägten Räumen durchgeführten, aber eher klein dimensionierten Einzelprojekten ausblieben.

Nach einer allgemeinen Bewertung der Kampagnenidee gefragt, bewerteten Anfang 2006 mehr als die Hälfte der Jugendlichen innovatives-oesterreich.at mit "sehr gut“ bzw. „gut" (Grafik 21). Ein weiteres Viertel benotete die Kampagne mit „,befriedigend“. Die Ergebnisse der zweiten Befragungswelle zeigen geringfügige Verschlechterungen in der Globalbewertung der Kampagne. So benoteten rd. $48 \%$ der Jugendlichen die Kampagne mit "sehr gut" bzw. "gut", die Note „befriedigend“ vergaben weitere $30 \%$. 


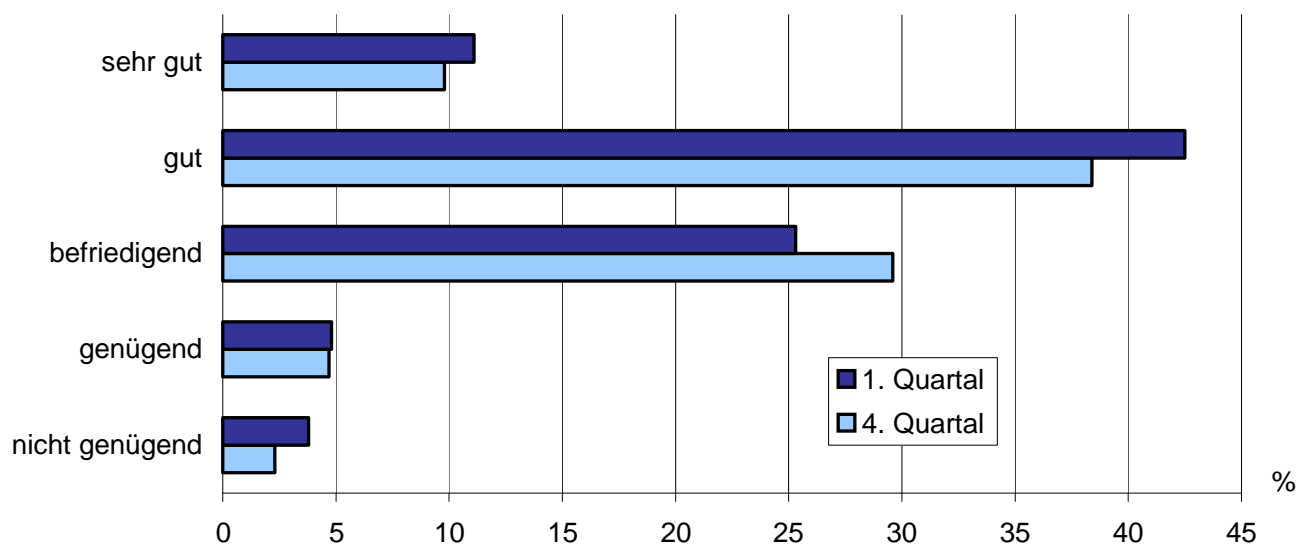

Anmerkungen:

N1=400, N4=403, Grundgesamtheit: Jugendliche 14-25 Jahre in Österreich (bevölkerungsrepräsentativ gewichtet)

Prozentanteile der Bewertungen anhand der Schulnotenskala von $1=$ sehr gut bis $5=$ nicht genügend, bezogen auf die Frage: „Und wie gefällt Ihnen die Idee der Kampagne innovatives-oesterreich ganz allgemein?"

Quelle: KMU FORSCHUNG AUSTRIA, TNS Infratest, Jugendlichen-Befragung 1. Quartal 2006; 4. Quartal 2006

Die Befragung hinsichtlich einer Detailbeurteilung der Kampagne innovatives-oesterreich.at ergibt folgendes Bild (Grafik 22): Im ersten und letzten Quartal 2006 sind zwischen 50 und $60 \%$ der befragten österreichischen Jugendlichen der Meinung, innovatives-oesterreich.at würde zum Nachdenken anregen, sei ein wichtiger Schritt für die Zukunft Österreichs, motiviere weitere Fragen zu stellen, würde junge Menschen ansprechen und wecke Neugier auf weitere Informationen zu diesem Thema. Etwas niedriger liegen die entsprechenden Werte hinsichtlich der Frage, inwieweit sie sich zum Besuch der Homepage motiviert sehen.

Grafik 22 Jugendliche: Kampagnenbeurteilung im Detail (1. Quartal 2006 / 4. Quartal 2006)

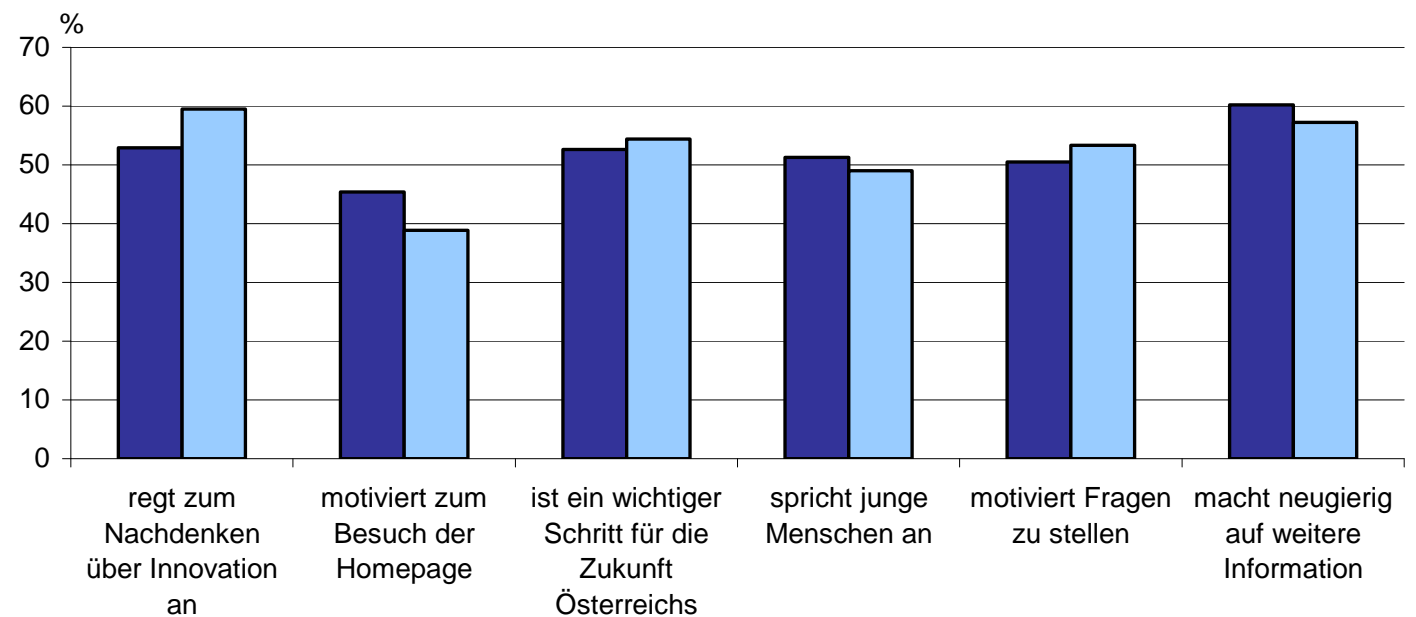

1. Quartal $\square$ 4. Quartal

Anmerkungen:

$\mathrm{N} 1=400, N 4=403$, Grundgesamtheit: Jugendliche 14-25 Jahre in Österreich (bevölkerungsrepräsentativ gewichtet)

Prozentanteile der Bewertungen „trifft voll zu“ und „trifft tw. zu“ bezogen auf „Wir haben hier einmal einige Detailaspekte gelistet, die auf eine solche Initiative mehr oder weniger zutreffen können. Bitte kreuzen Sie jeweils an, inwieweit diese Aussage auf die Kampagne innovatives-oesterreich.at zutrifft."

Quelle: KMU FORSCHUNG AUSTRIA, TNS Infratest, Jugendlichen-Befragung 1. Quartal 2006; 4. Quartal 2006 


\section{Exkurs: Werbewirkung der Kampagne innovatives-oesterrreich.at im internationalen Vergleich}

Für eine Einschätzung der Wirksamkeit der Kampagne innovatives-oesterreich.at ist ein Vergleich mit der Wirkung entsprechender Kampagnen von Bedeutung. Hierzu kann auf Messungen der Werbewirkungen von vergleichbaren Sensibilisierungskampagnen in Deutschland zurückgegriffen werden, die TNS Infratest mittels der AdEval-Methode durchgeführt hat. Gemessen werden hier die Zielgruppenansprache bzw. Überzeugungsleistung (Involvement) und die Motivationsleistung von Kampagnen (vgl. hierzu Grafik A-1 im Anhang). Hinsichtlich dieser beiden Messkriterien bildet die als Referenzmaßstab herangezogene Deutschland-Norm „Anzeigenkampagnen von Dienstleistern“ den Durchschnittswert entsprechender Wirkungstests von 85 Sensibilisierungskampagnen öffentlicher Auftraggeber in Deutschland ab.

Betrachtet man zunächst die Überzeugungs- und Motivationsleistung von innovativesoesterreich.at etwas genauer, so zeigt sich, dass im ersten Quartal $200649 \%$ der Befragten und im vierten Quartal $200643 \%$ der Befragten nach dem Betrachten eines Anzeigenmotivs der Kampagne eine bessere Meinung über innovatives-oesterreich.at hatten als vorher (vgl. Tabelle A-1).

Gemessen an dem genannten Referenzwert, wirkt innovatives-oesterreich.at überdurchschnittlich gut (Grafik 23). Wird die von TNS Infratest als Benchmark verwendete DeutschlandNorm herangezogen, so zeigt sich:

- innovatives-oestereich.at weist eine überdurchschnittlich hohe Zielgruppenansprache (Involvement) auf; diese liegt im ersten Quartal 2006 um $+16 \%$ und im vierten Quartal um $+11 \%$ höher als beim Durchschnitt vergleichbarer Kampagnen.

- Auch die Motivationsleistung von innovatives-oesterreich.at ist überdurchschnittlich hoch; sie liegt im ersten Quartal 2006 um $+12 \%$ und im vierten Quartal 2006 um $+6 \%$ über dem Durchschnitt vergleichbarer Kampagnen.

Grafik 23 Motivationsleistung (Markenimage) und Zielgruppenansprache (Involvement) der Kampagne innovatives-oesterreich.at, Jugendlichen-Befragung: Jugendliche gesamt

Abweichungen von der relevanten Norm ${ }^{\star}$ (in \%)

innovativesoesterreich.at (1. Qu. 2006) innovativesoesterreich.at (4. Qu. 2006)

Sensibilisierungskampagne in D

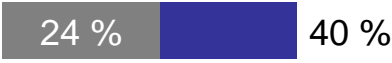

(*) Deutschland-Norm: 85 AdEval-Tests zu Anzeigenkampagnen von Dienstleistern

tns sinfratest Werbewirkungsforschung - AdEval ${ }^{\mathrm{TM}}$ total involvement

$58 \%$

$53 \%$

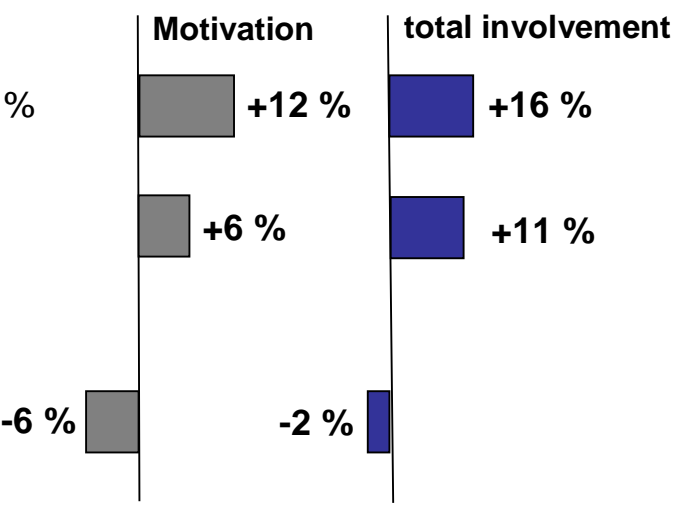

Quelle: KMU FORSCHUNG AUSTRIA / TNS infratest, Jugendlichen-Befragung 1. und 4. Quartal 2006 
Eine differenzierte Betrachtung zeigt insbesondere (Grafik 24), dass die Werbewirkung von innovatives-oesterreich.at bei den höher gebildeten Jugendlichen signifikant ausgeprägter ist als bei Jugendlichen mit niedrigerem formalem Bildungsstand. Zudem fällt auf, dass die Werbewirkung gegen Ende des Jahres 2006 leicht rückläufig ist. Dieser Umstand ist möglicherweise darauf zurückzuführen, dass bei der ersten Befragungswelle der „Werbedruck“ noch höher lag, da zu diesem Zeitpunkt insbesondere das "Großereignis“ Lange Nacht der Forschung noch nicht allzu lange zurücklag, während die Befragungswelle Ende 2006 in eine Zeit fiel, als die Aktivitäten im Rahmen von innovatives-oesterreich.at schon seit geraumer Zeit am „Abklingen“ waren. Dabei fällt auf, dass - mit Ausnahme der Motivationswerte unter den niedrig gebildeten Jugendlichen im vierten Quartal 2006 - die Motivationsleistung wie auch die Zielgruppenansprache der Kampagne innovatives-oesterreich.at auch im vierten Quartal 2006 nach wie vor über dem Durchschnitt des Referenzwertes liegt.

Grafik 24 Motivationsleistung und Zielgruppenansprache (Involvement) der Kampagne innovatives-oesterreich.at, Jugendlichen-Befragung: Jugendliche nach Geschlecht, Jugendliche nach Bildungsstatus

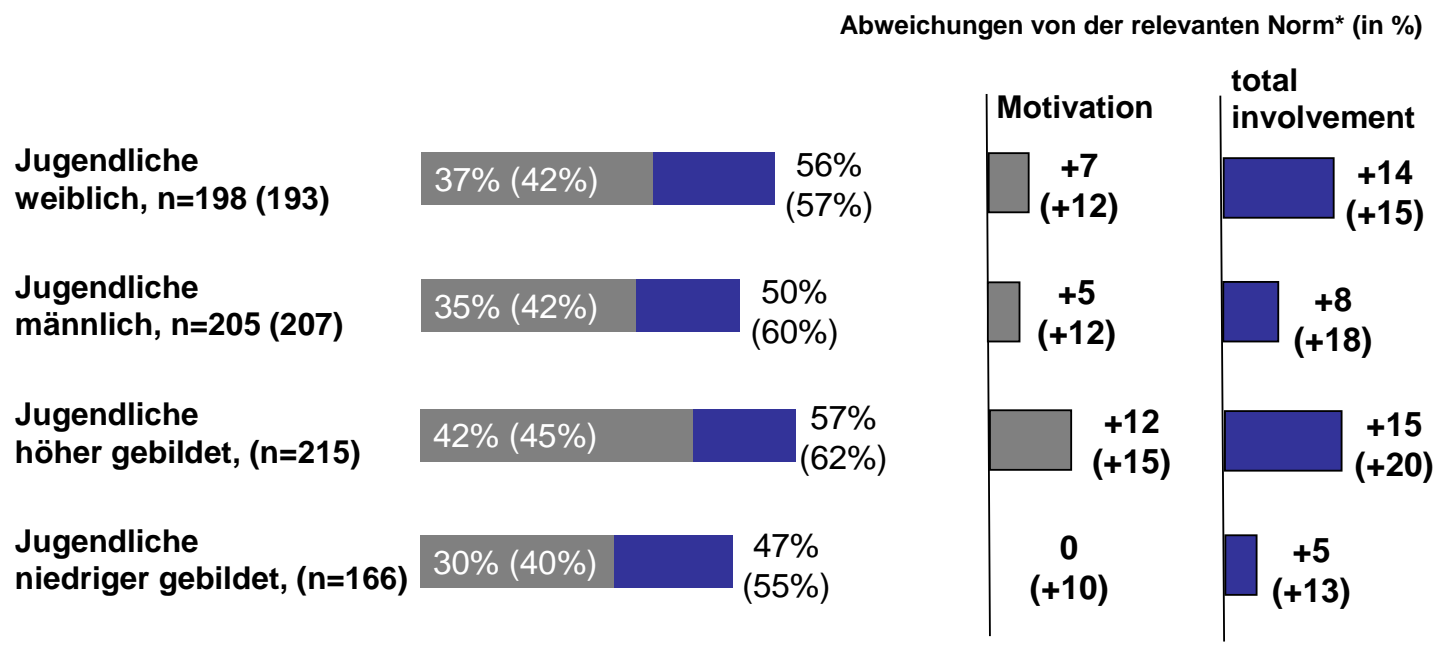

(*) Deutschland-Norm: 85 AdEval-Tests zu Anzeigenkampagnen von Dienstleistern

tns sinfratest Werbewirkungsforschung - AdEval ${ }^{\mathrm{TM}}$

Werte in Klammern: 1. Befragungswelle

Quelle: KMU FORSCHUNG AUSTRIA / TNS infratest, Jugendlichen-Befragung 1. und 4. Quartal 2006

Insgesamt deuten die überdurchschnittlich hohen Werte bei Involvement und Motivation darauf hin, dass die Kampagne innovatives-oesterreich.at bei der Zielgruppe Jugendliche offenkundig sowohl vom thematischen Zugang als auch von der Aufbereitung her überzeugen kann.

\subsection{Zielgruppe Klein- und Mittelunternehmen}

Die Wirkung der Kampagne innovatives-oesterreich.at bei der zweiten Zielgruppe - den Kleinund Mittelunternehmen - wurde ebenfalls an den beiden Zeitpunkten erstes und viertes Quartal 2006 gemessen. Es zeigt sich zunächst, dass Kampagne und Logo zu diesen Zeitpunkten bei etwa jedem fünften KMU bekannt waren (Grafik 25). Ein relevanter Unterschied nach Größe des Betriebes ist - abgesehen von der im vierten Quartal deutlich niedrigeren Bekanntheit der Kampagne bei den Mittelbetrieben - ist nicht zu beobachten. 


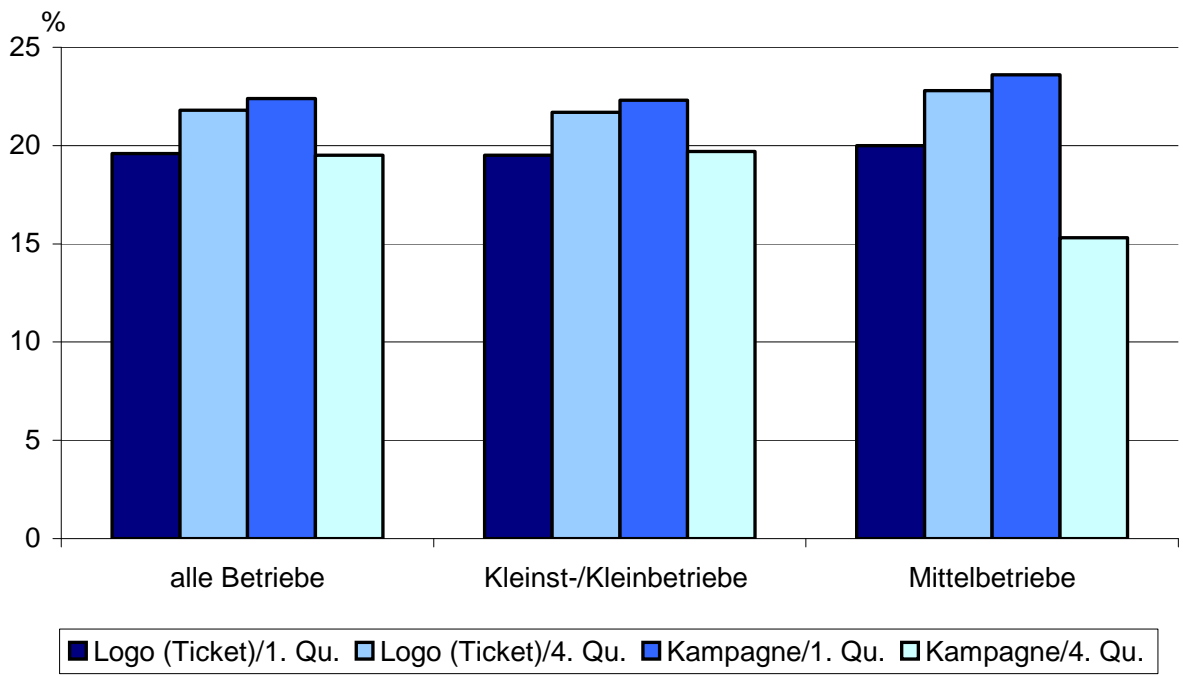

Anmerkungen:

$\mathrm{N} 1=3.552$, 3.682; N4=4.412, 4.501

1) Prozentanteil der Ja-Antworten auf die Fragen: „Kennen Sie die Kampagne innovatives-oesterreich.at?“; „Haben Sie das folgende Logo schon einmal in den Medien oder an anderer Stelle gesehen?"

$\left.{ }^{2}\right)$ Kleinst-/Kleinbetriebe: Unternehmen mit 1-49 Beschäftigten, Mittelbetriebe 50-249 Beschäftigte

Quelle: KMU FORSCHUNG AUSTRIA, KMU-Befragung 1. Quartal 2006 / 4. Quartal 2006

Wird bezüglich der Bekanntheitswerte zwischen innovationsorientierten und nicht-innovativen KMU unterschieden ${ }^{25}$, ergibt sich ein durchaus erwarteter Befund (Grafik 26): Innovationsorientierten Unternehmen, also solchen Unternehmen, die in den letzten drei Jahren vor der Befragung Produkt- und/oder Prozessinnovationen durchgeführt hatten, ist sowohl die Kampagne als auch das Logo häufiger bekannt als ihren nicht-innovationsaktiven Pendants; dieser angesichts der eher auf Innovation ausgerichteten Geschäftsaktivitäten naheliegender Vorsprung an Informiertheit beläuft sich bei den einzelnen Bekanntheitswerten von Kampagne, Logo und Lange Nacht der Forschung auf 6 bzw. 8 Prozentpunkte.

25 Eine solche Unterscheidung ist nur auf Basis der Werte der 1. Befragungswelle möglich. 

„innovatives-oesterreich.at“ und der „Langen Nacht der Forschung“ (1. Quartal 2006)

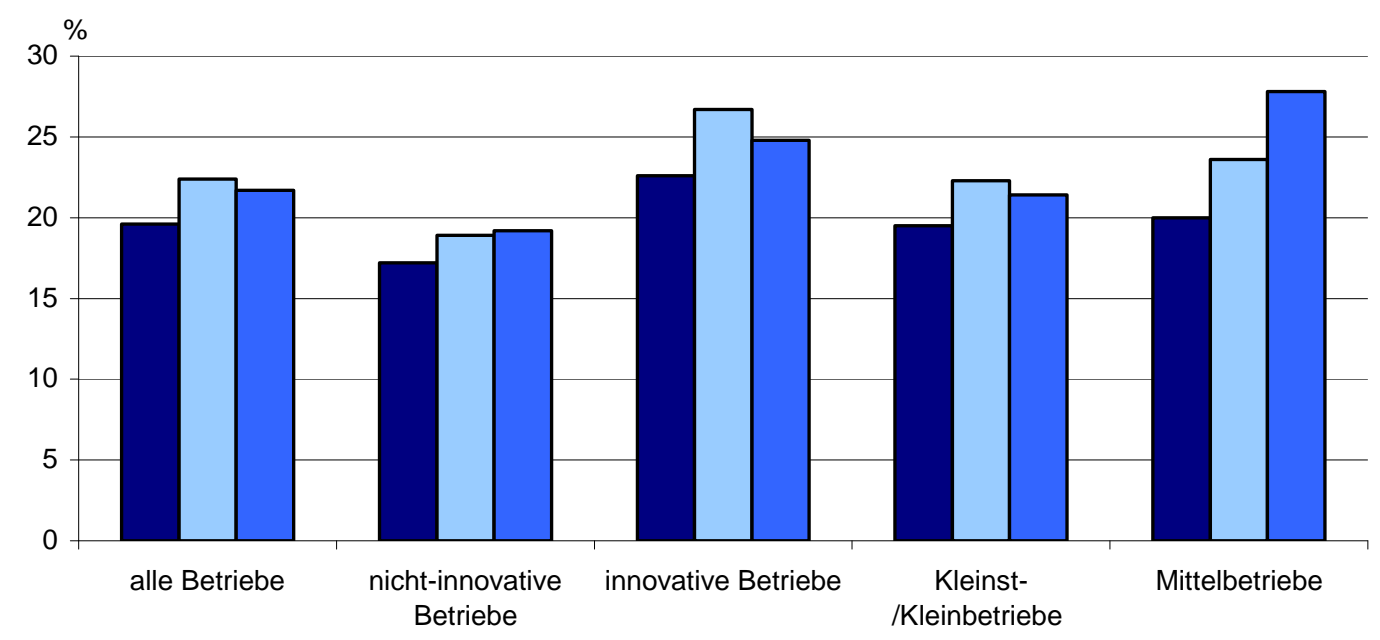

Q Logo (Ticket) $\quad$ KKampagne $\quad$ LLNDF

Anmerkungen: $\mathrm{N}=3.682,3.552,3.496$

1) Prozentanteil der Ja-Antworten auf die Fragen: „Kennen Sie die Kampagne innovatives-oesterreich.at?“ bzw. „Kennen Sie die Lange Nacht der Forschung?“, „Haben Sie das folgende Logo schon einmal in den Medien oder an anderer Stelle gesehen?"

$\left.{ }^{2}\right)$ „nicht-innovative Betriebe“: Unternehmen, die in den letzten drei Jahren keine Produkt- und/oder Prozessinnovationen durchgeführt haben; „innovative Betriebe“: Unternehmen, die in den letzten drei Jahren Produkt- und/oder Prozessinnovationen durchgeführt haben

Quelle: KMU FORSCHUNG AUSTRIA, KMU-Befragung 1. Quartal 2006

Der Hälfte der befragten KMU gefällt die Kampagnenidee „gut“ oder „sehr gut“; dies gilt sowohl für das erste als auch für das vierte Quartal 2006 (Grafik 27). Weitere knapp $40 \%$ benoteten die Kampagne mit „befriedigend“.

\section{Grafik 27 KMU: Kampagnenbeurteilung global (1. Quartal 2006 / 4. Quartal 2006)}

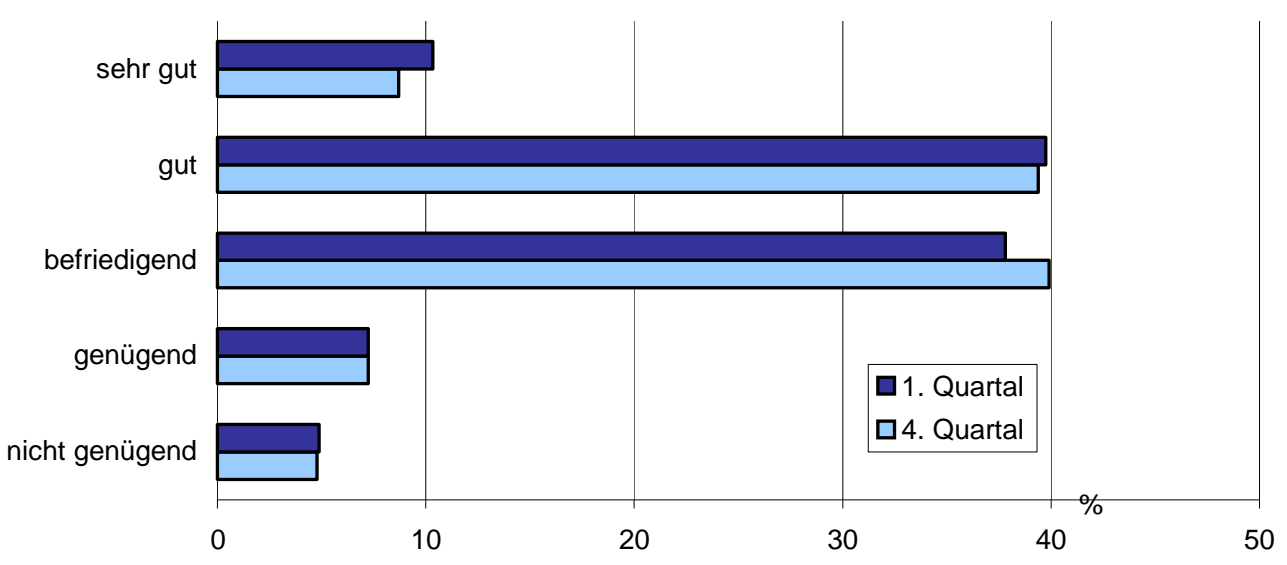

Anmerkungen: $\mathrm{N} 1=2.156 ; \mathrm{N} 4=2.739$

Prozentanteile der Bewertungen anhand der Schulnotenskala von $1=$ sehr gut bis $5=$ nicht genügend, bezogen auf die Frage: „Die Kampagne innovatives-oesterreich.at soll das Verständnis für Innovation und Forschung erhöhen. Sie soll Innovations- und Forschungsaktivitäten anregen und eine Plattform für den Dialog zu Innovation, Forschung und Technologieentwicklung darstellen. Wie gefällt Ihnen die Kampagne bzw. die Idee?"

Quelle: KMU FORSCHUNG AUSTRIA, KMU-Befragung 1. Quartal 2006; 4. Quartal 2006 
Auch bei der Bewertung von Detailaspekten der Kampagne zeigt sich kein gravierender Unterschied zwischen erstem und letztem Quartal 2006 (Grafik 28): Jeweils etwa $80 \%$ der befragten KMU gaben an, dass aus ihrer Sicht die Kampagne innovatives-oesterreich.at zum Nachdenken über Innovationen anregt, junge Menschen anspricht und insgesamt einen wichtigen Schritt für die Zukunft Österreichs darstellt. Etwas weniger häufig verbreitet ist - ähnlich wie bei den entsprechenden Bewertungen der Jugendlichen - die Ansicht, die Kampagne motiviere zum Besuch der Homepage, um dort weitere Informationen zu erhalten.

Grafik 28 KMU: Kampagnenbeurteilung im Detail (1. Quartal 2006 / 4. Quartal 2006)

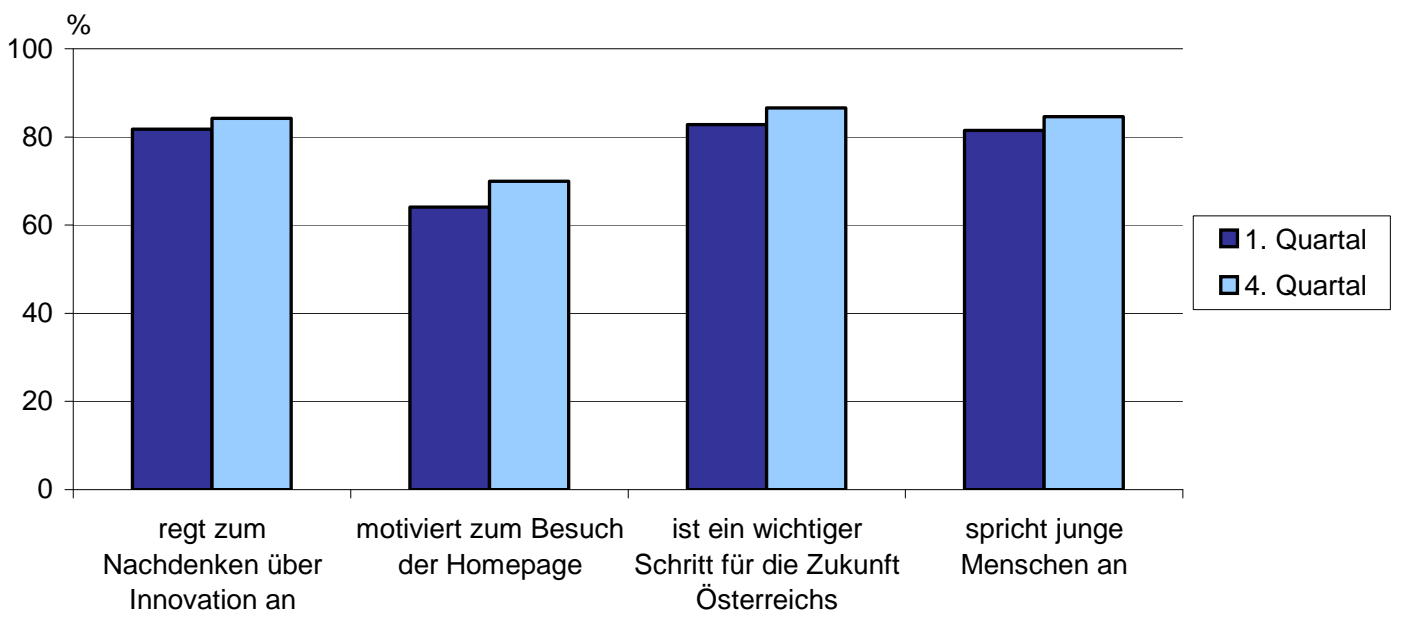

Anmerkungen:

$\mathrm{N} 1=2.020,1.988,2.009,2.006 ; \mathrm{N} 4=480,489,478,483$

Prozentanteile der bezogen auf die Frage: „Bitte kreuzen Sie zu jedem der genannten Teilaspekte an, ob er aus Ihrer Sicht auf die Kampagne innovatives-oesterreich.at zutrifft ...". Antworten „trifft voll zu“ und "trifft teilweise zu“

Quelle: KMU FORSCHUNG AUSTRIA, KMU-Befragung 1. Quartal 2006 / 4. Quartal 2006

\subsection{Das „Vorzeigeprojekt“ Lange Nacht der Forschung 2005}

Im Rahmen der begleitenden Evaluierung wurde auch eine Wirkungsanalyse der ersten Langen Nacht der Forschung in Österreich durchgeführt; dieses „Großereignis“ im Rahmen der zweiten Kampagnenphase hat am 1. Oktober 2005 an den drei Standorten Wien, Linz und Innsbruck stattgefunden und nach Zählung der Veranstalter etwa 48.000 Besucher/innen gehabt. Übergeordnetes Thema der Veranstaltung war „Alles im Griff? Wissenschaft und Sicherheit“. Es beteiligten sich Universitäten, außeruniversitäre Forschungseinrichtungen und Unternehmen, die einzelne Stationen an den drei Standorten betreuten.

Die Ergebnisse der Wirkungsanalyse der Langen Nacht der Forschung 2005 werden hier etwas ausführlicher dargestellt. Dies erscheint insbesondere deshalb sinnvoll zu sein, da für die kommenden Jahre ähnliche Veranstaltungen geplant sind. Die hier vorgestellten Ergebnisse zur Wirkung der Langen Nacht der Forschung basieren auf der Befragung von Besucher/innen und Veranstalter/innen sowie auf den Ergebnissen der teilnehmenden Beobachtung (vgl. zur Vorgehensweise im Detail Kapitel 1).

\subsubsection{Ergebnisse der Besucher/innenbefragung}

Gegenstand der Besucher/innenbefragung waren neben der Aufnahme von soziodemografischen Merkmalen Fragen zu Informationswegen, Motivation, Interessenschwerpunkten, Zufriedenheit und Bewertungen sowie allgemeine Einschätzungen zu Forschung und Innovation in Österreich. 
Die Auswertung nach soziodemografischen Merkmalen zeigt: Die Besucher/innen der Langen Nacht der Forschung 2005 waren jung und gut ausgebildet. Das Durchschnittsalter der befragten Besucher/innen lag bei 31 Jahren $^{26}$, der Anteil von Kindern und Jugendlichen (bis 21 Jahre) betrug $32 \%$. Neben einer ausgewogenen Geschlechterverteilung (49\% weibliche und $51 \%$ männliche Besucher) fällt auf, dass hinsichtlich des Ausbildungshintergrundes überwiegend Personen mit einem höheren Bildungsabschluss bzw. Ausbildungsgang vertreten waren: Der am stärksten vertretene Ausbildungshintergrund der Besucher/innen wurde mit Matura (40\%) angegeben, gefolgt von Personen mit einem Hochschul- oder Fachhochschulabschluss (33 \%); einen Pflichtschulabschluss hatten $12 \%$, eine duale Berufsausbildung $10 \%$ der Besucher/innen abgeschlossen. $43 \%$ der Befragten waren zum Zeitpunkt der Erhebung Arbeitnehmer/innen, $25 \%$ Student/innen, $17 \%$ Schüler/innen und $10 \%$ Selbständige. Im Durchschnitt haben die Besucher/innen an fünf Veranstaltungen der Langen Nacht der Forschung teilgenommen.

Die Besucher/innen der Langen Nacht der Forschung 2005 sind generell sehr positiv zu Aktivitäten der Öffentlichkeitsarbeit für die Bereiche Wissenschaft und Forschung eingestellt; so halten $86 \%$ der Befragten eine diesbezogene Öffentlichkeitsarbeit für notwendig. Auf den ersten Blick naheliegend, mit Blick auf die oben skizzierten Probleme bei der Verwendung der Dachmarke gerade bei der Langen Nacht der Forschung 2005 (vgl. Kapitel 4.5) aber doch bemerkenswert ist der Umstand, dass $86 \%$ der befragten Besucher/innen der Langen Nacht der Forschung die Kampagne innovatives-oesterreich.at bekannt war - ein hoher Wert, selbst wenn der bei solchen Umfragen generell in Rechnung zu stellende Bias der sozialen Erwünschtheit im Antworthalten berücksichtigt wird.

Betrachtet man die Informationswege, über die Besucher/innen von der Langen Nacht der Forschung erfahren haben, so lässt sich aus den Antworten eine gewisse Dominanz sozialer Kontakte und eher traditioneller Informationswege herauslesen: Ein Drittel der Besucher/innen gibt an, dass Freunde und Bekannte sie auf die Veranstaltung hingewiesen haben; $28 \%$ sind über TV und Radio, $24 \%$ über Plakate und $21 \%$ über Zeitungsartikel auf die Veranstaltung aufmerksam geworden (Mehrfachnennungen waren möglich). Erst danach rangiert mit $18 \%$ der Nennungen das Internet. Auffällig ist zudem, dass lediglich $5 \%$ der Besucher/innen angeben, über die Schule von der Langen Nacht der Forschung erfahren zu haben - angesichts des relativ hohen Anteils an Schüler/innen an den Besucher/innen eine recht niedrige (und verbesserungsfähige) Quote.

Die vorrangige Motivation für den Besuch der Langen Nacht der Forschung 2005 war offenkundig eher allgemeiner Natur (Grafik 29): $74 \%$ der Besucher/innen geben an, aus Neugierde gekommen zu sein. Bemerkenswert ist allerdings, dass etwa $55 \%$ der Besucher/innen ein Interesse an neuen Trends in Forschung und Technologie als Grund für den Besuch der Langen Nacht nennen. Der Anteil der Besucher/innen, die ihren Besuch mit einem gezielten Interesse begründen, liegt deutlich niedriger: Als Motiv für den Besuch geben $22 \%$ der Besucher/innen berufliches Interesse an, $13 \%$ Informationsbedarf zu möglichen Ausbildungswegen und $11 \%$ einen anderen speziellen Informationsbedarf.

26 Und schwankte dabei zwischen 9 und 73 Jahren. 


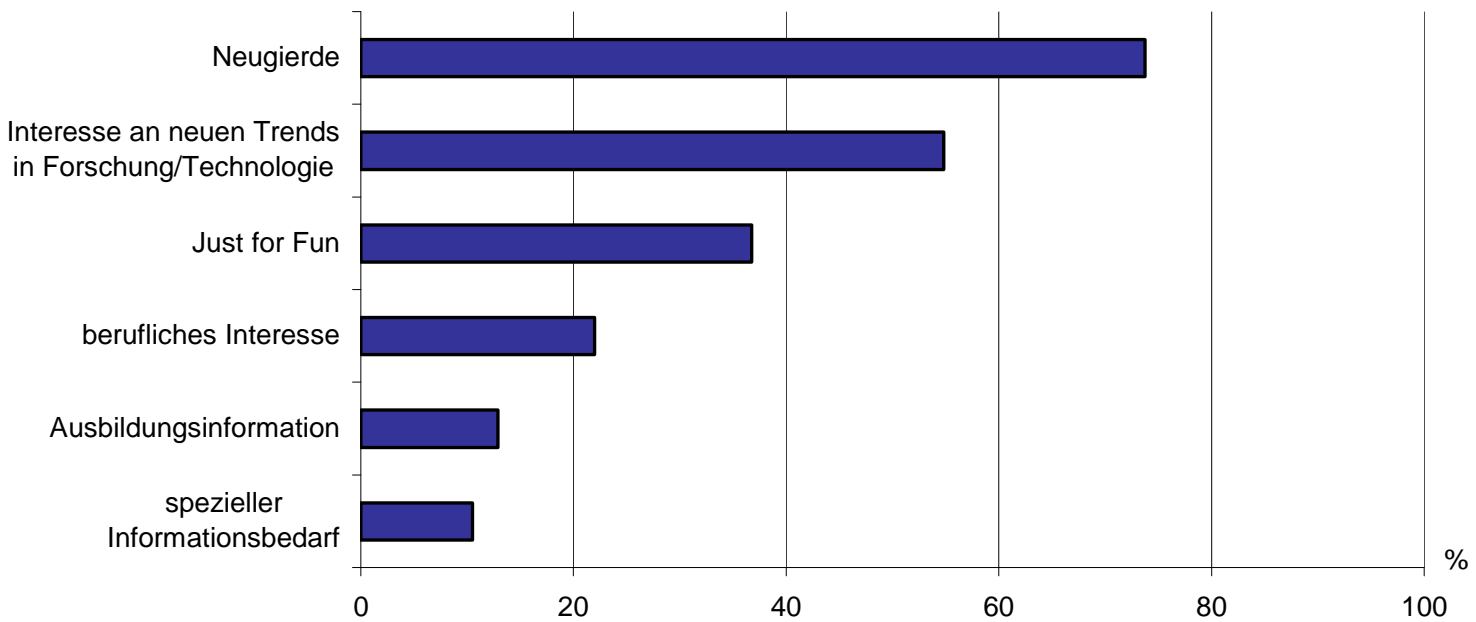

Anmerkungen:

$\mathrm{N}=914$

*) Mehrfachantworten möglich

Quelle: KMU FORSCHUNG AUSTRIA, Besucher/innenbefragung (LNdF 2005)

Das Hauptinteresse des überwiegenden Teils der Besucher/innen liegt in den ingenieurwissenschaftlichen und naturwissenschaftlichen Bereichen (Grafik 30): An erster Stelle steht das Interesse an den Ingenieurwissenschaften und Neuen Technologien (45\%); Je etwa $40 \%$ der Besucher/innen äußern Interesse an den Bereichen Geo-, Weltraum- und Verkehrsforschung, Mathematik, Informatik, Physik, Chemie und Biologie, $37 \%$ Interesse an medizinischer Forschung. Interesse an den Sozial- und Geisteswissenschaften äußern 29 \% der Besucher/innen.

Grafik $30 \quad$ Lange Nacht der Forschung 2005: Besonderes Interesse der Besucher/innen an wissenschaftlichen Bereichen

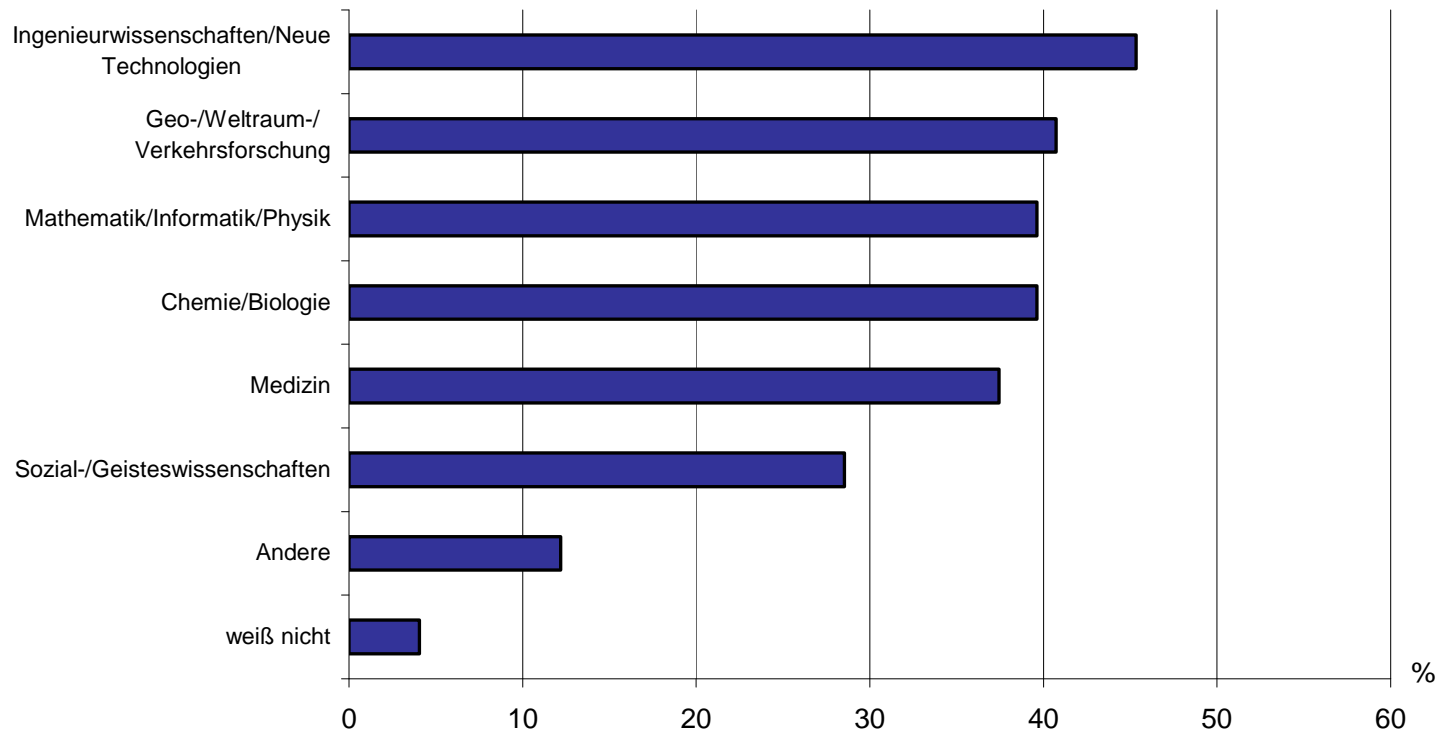

Anmerkungen: $\mathrm{N}=911$

Mehrfachantworten möglich

Quelle: KMU FORSCHUNG AUSTRIA, Besucher/innenbefragung (LNdF 2005) 
Wird die Zufriedenheit der Besucher/innen mit verschiedenen Aspekten der Langen Nacht der Forschung 2005 betrachtet, so fällt die außerordentlich positive Bewertung auf (Grafik 31): Jeweils über $90 \%$ der Besucher/innen haben einen positiven Gesamteindruck bzw. sind mit der Anzahl der Veranstaltungen, dem Neuigkeitswert der Inhalte und der Verständlichkeit der Präsentationen „zufrieden“ oder gar „sehr zufrieden“. Bemerkenswert ist dabei auch, dass die Kommunikation zwischen Wissenschaftler/innen und Besucher/innen offenkundig "funktioniert" hat: Hierauf deutet neben der positiven Bewertung der Verständlichkeit der Präsentationen durch die Wissenschaftler/innen auch die Tatsache hin, dass $86 \%$ der Besucher/innen auch mit Möglichkeit, mit Expert/innen zu diskutieren, „zufrieden“ oder „sehr zufrieden“ waren - angesichts häufig erwähnter Schwierigkeiten der Wissenschaftskommunikation erstaunlich hohe Werte, die noch einer differenzierteren Analyse bedürfen. $80 \%$ der Befragten sehen zudem auch die Angebote für Jugendliche und Kinder positiv, was insbesondere mit Blick auf die Zielgruppe „Jugendliche“ der Kampagne www.innovatives-oesterreich.at von Bedeutung ist. Auch die Organisation der Langen Nacht der Forschung, die insbesondere bei den Veranstalter/innen auf Kritik stieß, erreicht bei den Besucher/innen noch einen "Zufriedenheitsgrad“ von $70 \%$. Betrachtet man die real existierenden organisatorischen Probleme, die vor Ort insbesondere mit dem Shuttle-Pendelverkehr existierten, so kann dieser Wert als eine gewisse „Robustheit“ der am Thema interessierten Öffentlichkeit gegenüber organisatorischer bedingter "Unbequemlichkeit" ausgelegt werden.

Alles in allem sind dies, selbst wenn man von einem gewissen Bias dahingehend ausgeht, dass die Antwortbereitschaft bei den „positiv gestimmte Personen“ überdurchschnittlich hoch ausgeprägt war, beachtlich hohe Zufriedenheitswerte. Dieser Eindruck wird dadurch bestätigt, dass $55 \%$ der Besucher/innen eine Lange Nacht der Forschung auf jeden Fall wiederbesuchen würden, und $73 \%$ auf jeden Fall eine Weiterempfehlung zum Besuch dieser Veranstaltung aussprechen.

Grafik $31 \quad$ Lange Nacht der Forschung 2005: Zufriedenheit der Besucher/innen, Global und Detailaspekte

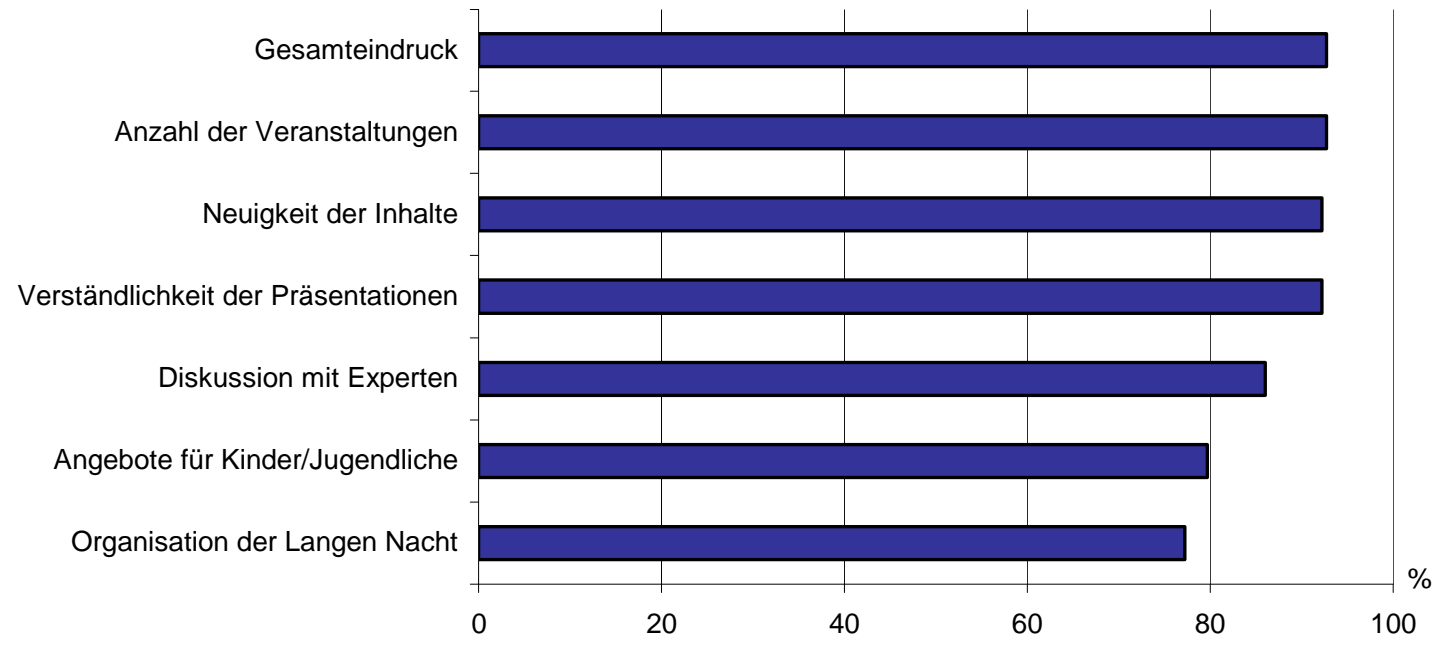

Anmerkungen:

$\mathrm{N}=695$ bis 893

Antwortkategorien „sehr zufrieden“ und „zufrieden“

Quelle: KMU FORSCHUNG AUSTRIA, Besucher/innenbefragung (LNdF 2005)

\subsubsection{Ergebnisse der Veranstalter/innenbefragung}

Die Veranstalter/innen wurden nach ihrem Zugang zur Teilnahme an der Langen Nacht der Forschung, ihren Motiven und Erwartungen, dem Veranstaltungstyp, dem erforderlichen Aufwand sowie nach ihrer Zufriedenheit mit Vorbereitung, Betreuung und Durchführung befragt. Darüber 
hinaus wurden sie um eine Einschätzung gebeten, welchen Nutzen ihre Institution aus der Teilnahme möglicherweise ziehen kann und welche mittelfristigen Effekten sie erwarten.

Vorrangige Motive für die Teilnahme an der Langen Nacht der Forschung 2005 waren für die Veranstalter/innen und Betreuer/innen Verbesserungen in der Kommunikation ihrer Arbeit gegenüber der Öffentlichkeit (Grafik 32). Für je $68 \%$ der Befragten war die Kommunikation von Wissenschaft und Forschung nach außen und die Verbesserung der Sichtbarkeit der eigenen Forschungsarbeit in der Öffentlichkeit ein als „sehr wichtig“ eingestuftes Motiv. Einen Beitrag zur Steigerung der gesellschaftlichen Akzeptanz von Wissenschaft und Forschung zu leisten, war für über die Hälfte der Befragten sehr wichtig. Die Rekrutierung von potenziellem Forschungsnachwuchs wurde von $4 \%$ als „sehr wichtig“, von $29 \%$ als zumindest „wichtig“ eingestuft; die monetäre Förderung war für $9 \%$ „sehr wichtig“ und für $21 \%$ immerhin „wichtig“ für die Teilnahme. $5 \%$ der befragten Veranstalter/innen sahen sich in irgendeiner Form verpflichtet, an der Langen Nacht der Forschung teilzunehmen.

Grafik 32

Lange Nacht der Forschung 2005: Motive der Veranstalter/innen für die Teilnahme

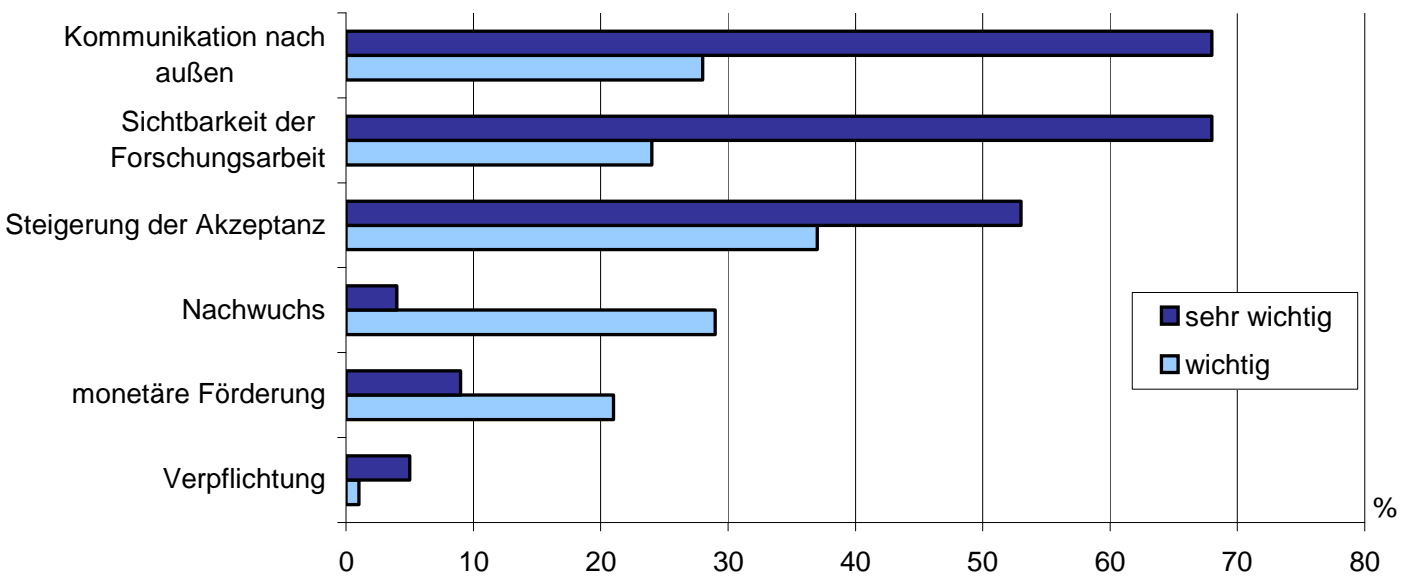

Anmerkungen:

$\mathrm{N}=75$ (111 Stationen)

Quelle: KMU FORSCHUNG AUSTRIA, Veranstalter/innenbefragung (LNdF 2005)

Den Gesamtaufwand, den die Veranstalter/innen für die Teilnahme an der Langen Nacht der Forschung treiben mussten, wird von $67 \%$ der befragten Veranstalter/innen als angemessen beurteilt; etwa $25 \%$ empfanden den Aufwand als zu hoch, $5 \%$ als deutlich zu hoch.

Die Arbeit der Organisatoren bei der Vorbereitung der Langen Nacht der Forschung wird wie folgt beurteilt (Grafik 33): Auf einer Skala von 1 („,sehr zufrieden“) bis 4 („gar nicht zufrieden“) werden die Aspekte Erreichbarkeit der Organisatoren und Auswahl der Medienpartner leicht überdurchschnittlich gut bewertet. Im Bereich einer durchschnittlichen bzw. befriedigenden Bewertung bewegen sich, in abnehmendem Grad, die Kompetenz der Organisatoren, der administrative Aufwand für die Antragstellung und auch noch die 


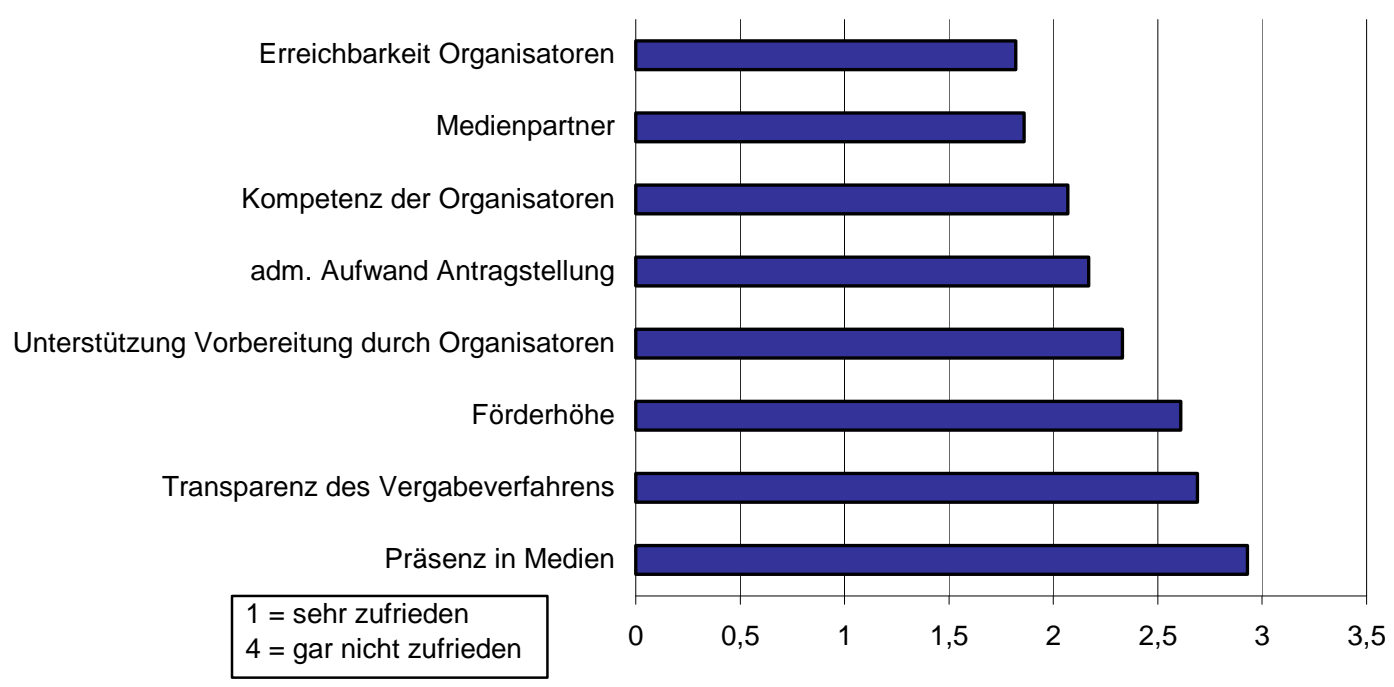

Anmerkungen:

$\mathrm{N}=75$ (111 Stationen)

Quelle: KMU FORSCHUNG AUSTRIA, Veranstalter/innenbefragung (LNdF 2005)

Unterstützung bei der Vorbereitung der Langen Nacht durch die Organisatoren. Kritischer werden hingegen die Aspekte Höhe der Förderung, Transparenz des Vergabeverfahrens sowie insbesondere die Präsenz der Langen Nacht der Forschung in den Medien gesehen, mit der man sich „eher wenig zufrieden“ zeigte.

Als primären Nutzen und wichtige weitere Effekte der Teilnahme erwarten sich die Veranstalter/innen, dass sie auf diesem Wege eine Verbesserung der Sichtbarkeit der eigenen Forschungsarbeit in der Öffentlichkeit erreichen (Grafik 34); $92 \%$ der Veranstalter/innen betrachten dies als einen „wichtigen“ oder "sehr wichtigen“ Nutzen der Teilnahme für ihre Institution. Knapp 80 \% erwarten eine Erhöhung der Akzeptanz ihrer Forschungsarbeit, 64 \% gehen davon aus, dass sie mit ihrer Präsentation das Interesse von Jugendlichen wecken.

Immerhin mehr als die Hälfte der Veranstalter/innen erwartet offenkundig, dass ihre Teilnahme einen Kompetenzaufbau im Bereich der Vermittlung von Forschungsthemen für die Öffentlichkeit bewirkt: So geben $55 \%$ der Veranstalter/innen an, dass sie anlässlich der Teilnahme an der Langen Nacht der Forschung ihre Präsentationsunterlagen weiterentwickelt haben. In diesem Zusammenhang bekunden $21 \%$ der Veranstalter/innen, dass ihre Teilnahme an der Langen Nacht der Forschung den Einstieg ihrer Institution in die systematische Öffentlichkeit bedeutet.

Gegenüber diesen kommunikativen Aspekten sind die Erwartungen hinsichtlich eines Nutzen der Teilnahme an der Langen Nacht der Forschung für die wissenschaftliche Arbeit eher begrenzt: $37 \%$ der Veranstalter/innen geben an, dass die Teilnahme die Möglichkeit zu neuen und interessanten Kontakten bietet, $28 \%$ der Befragten erhoffen sich eine verbesserte Kooperation mit anderen Veranstalter/innen am Standort. Angesichts der Zielsetzung der Langen Nacht der Forschung erscheinen diese Werte bzw. die darin zum Ausdruck kommenden Erwartungen eines „Teilnahme-Nutzens“ für die wissenschaftliche bzw. Forschungsarbeit eher als überraschend hoch. 

Institutionen aus Sicht der Veranstalter/innen

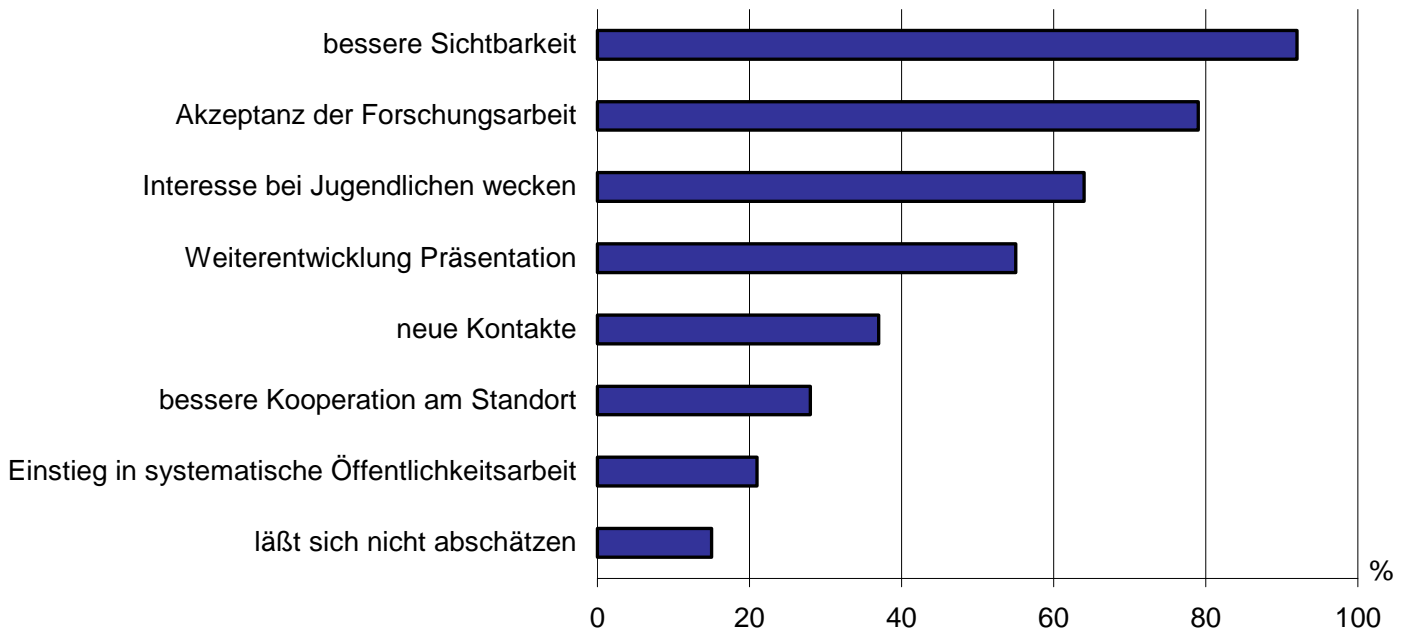

Anmerkungen:

$\mathrm{N}=75$ (111 Stationen)

Quelle: KMU FORSCHUNG AUSTRIA, Veranstalter/innenbefragung (LNdF 2005)

Insgesamt betrachtet fällt die Bewertung der Langen Nacht der Forschung 2005 durch die Veranstalter/innen eher positiv aus (Grafik 35): $32 \%$ der Befragten sehen im Nachhinein ihre Erwartungen an die Lange Nacht der Forschung deutlich übertroffen, mehr als die Hälfte der Veranstalter/innen sieht die Erwartungen als erfüllt an. $56 \%$ der Veranstalter/innen würden im nächsten Jahr „auf jeden Fall“ nochmals eine Station im Rahmen einer neu aufgelegten Langen der Forschung betreuen. Weitere $28 \%$ der Veranstalter/innen bejahen dies ebenfalls, fügen aber hinzu, dass dies nur unter anderen Bedingungen vorstellbar sei. Es liegt nahe, in diesem letztgenannten Wert noch erhebliche Verbesserungspotenziale bei der Vorbereitung, Betreuung und Durchführung zu vermuten.

Sehr gut fällt auch die Bewertung der Langen Nacht der Forschung 2005 durch die Besucher/innen aus. $55 \%$ der Befragten können sich auf jeden Fall einen erneuten Besuch einer Langen Nacht der Forschung vorstellen und nahezu Dreiviertel der befragten Besucher/innen wollten die Lange Nacht auf jeden Fall weiterempfehlen. 

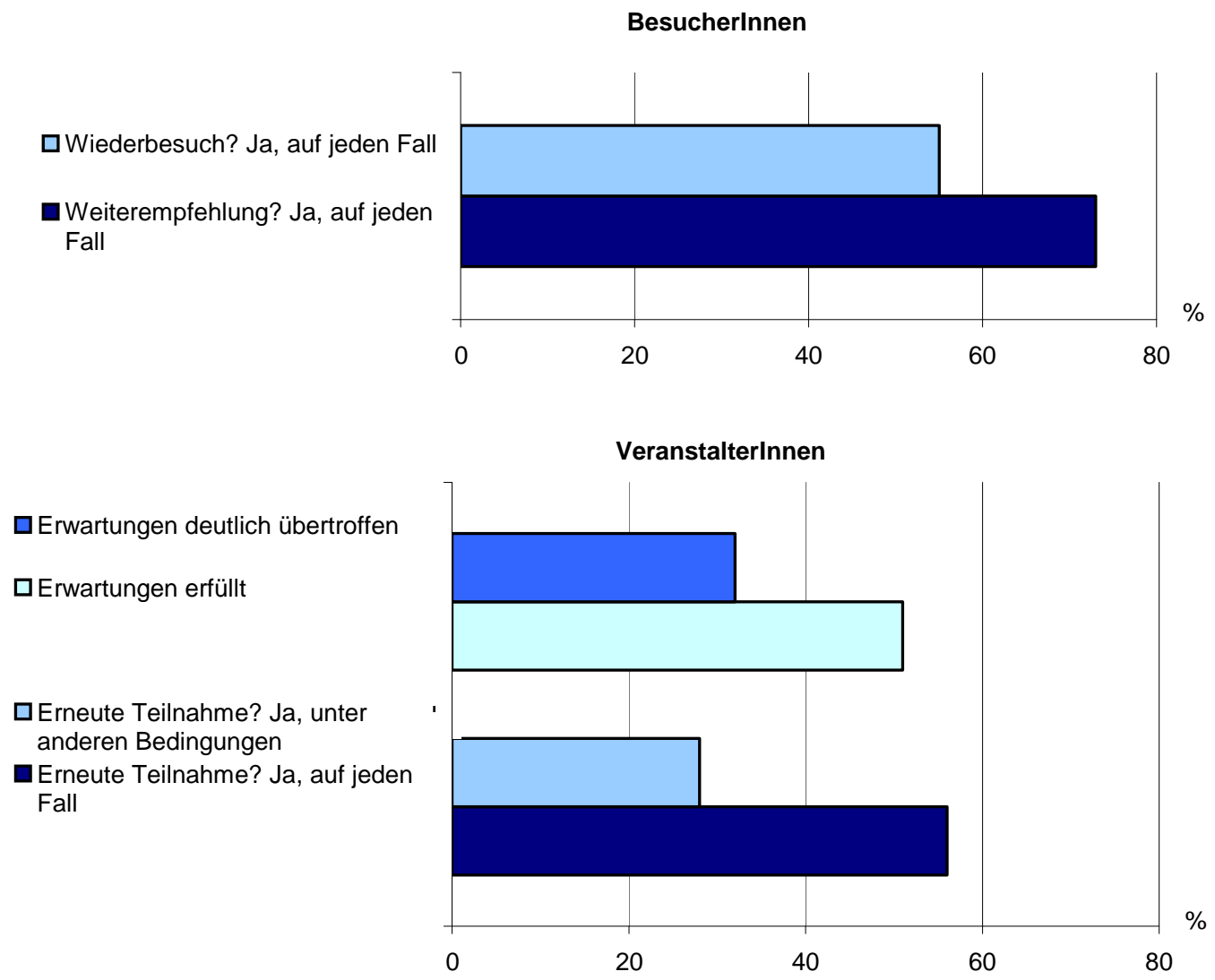

Anmerkungen:

N (Besucher/innen)=870; 867; N (Veranstalter/innen)=75 (111 Stationen)

Quelle: KMU FORSCHUNG AUSTRIA, Besucher/innenbefragung; Veranstalter/innenbefragung (LNdF 2005)

\subsubsection{Verbesserungspotenziale}

Folgende Anmerkungen beziehen sich im Wesentlichen auf die Vor- und Nachbereitung einer solchen Veranstaltung, darüber hinaus auch auf die praktisch-organisatorische Gestaltung. Grundlage ist eine Auswertung der abschließenden offenen Frage, die den Veranstalter/innen Gelegenheit zu kritischen Kommentaren, Verbesserungsvorschlägen u.ä. gab.

Verbesserungspotenziale hinsichtlich der Vorbereitung:

- kritisiert wurde von den Veranstalter/innen bzw. Betreuer/innen eine mangelnde Betreuung durch die Organisatoren der Langen Nacht der Forschung;

- bemängelt wurde - insbesondere von Seiten universitärer Veranstalter/innen - eine fehlende Einbindung der Veranstalter/innen in die Vorbereitung; in diesem Zusammenhang gibt es Vorschläge, die Organisation einer solchen Veranstaltung dezentral durchzuführen

- kritisiert wurde die mangelnde Transparenz im Vergabeverfahren

- die Vorbereitungszeit wurde vielfach als zu knapp angesehen;

- relativ häufig wurde eine unzureichende PR im Vorfeld der Langen Nacht der Forschung kritisiert; 
- vorgeschlagen wird, die Terminwahl so vorzunehmen, dass einerseits Student/innen besser einbezogen werden können, andererseits keine zeitliche Nähe zu ähnlich gelagerten Veranstaltungen mit entsprechenden Demobilisierungseffekten gegeben ist;

Verbesserungspotenziale hinsichtlich der praktisch-organisatorischen Seite:

- an prominenter Stelle wurde der Bustransfer als völlig unzureichend kritisiert; gefordert werden knappere Intervalle und bessere Ausschilderungen - angesichts des unerwartet hohen Besucherandrangs eine offenkundig weitgehend unstrittige Verbesserungsnotwendigkeit;

- gewünscht wird ein besseres Briefing der Helfer vor Ort;

- selbstkritisch wurde von nicht wenigen Veranstalter/innen darauf hingewiesen, dass sie die Notwendigkeit der Überarbeitung ihrer Präsentationsunterlagen / -methoden sehen;

Verbesserungspotenziale in der Nachbereitung:

- vermisst wurde vielfach eine bessere Nachbereitung der Veranstaltung, die etwa im Rahmen eines entsprechend Meetings der Veranstalter/innen an den einzelnen Standorten stattfinden könnte

\subsection{Die Einzelprojekte}

Die Befragung der Projektträger/innen vermittelt den Eindruck, dass die unter dem Dach von innovatives-oesterreich.at durchgeführten Projekte die Zielgruppen offenkundig insgesamt gut erreichen; relevante Probleme werden diesbezüglich auch im Rahmen der qualitativen Erhebungen nicht genannt. 15 Projektträger/innen von zum Zeitpunkt der Online-Befragung, im Frühjahr 2006, bereits „absolvierten“ Projektaktivitäten geben an, ihr Zielpublikum „auf jeden Fall“ erreicht zu haben, die restlichen 5 beantworten diese Frage zumindest mit „eher ja“.

Bei der Einschätzung über die Erwartungen von Teilnehmer/innen einzelner Projektaktivitäten steht offensichtlich der Aspekt des Dialogs im Vordergrund. Besucher/inne und Teilnehmer/innen von Projektaktivitäten im Rahmen der erwarteten sich offenkundig vorrangig Diskussionsmöglichkeiten und Kontakt zu Expert/innen sowie spezielle und fachorientierte Informationen womit der Grundcharakter der zweiten Kampagne, den Dialog zwischen Forschung und Öffentlichkeit zu forcieren, unterstrichen wird. Für 15 bzw. 14 Projektträger/innen trifft die Erwartung seitens der Besucher/innen mit Expert/innen Kontakt aufzunehmen oder zu diskutieren bzw. spezielle/fachorientierte Informationen zu erfahren, sehr zu (Grafik 36). Der Besuch bzw. die Teilnahme an durchgeführten Projektaktivitäten aus allgemeinem Interesse an Wissenschaft und Forschung trifft für sieben Projektträger/innen „sehr“ zu, für weitere acht trifft dies „eher" zu. Informationen zu Ausbildungsmöglichkeiten und reine Unterhaltung sind nur für jeweils vier Projektträger/innen primäre Erwartungen an Projektaktivitäten seitens der $\mathrm{Be}$ sucher/innen. 


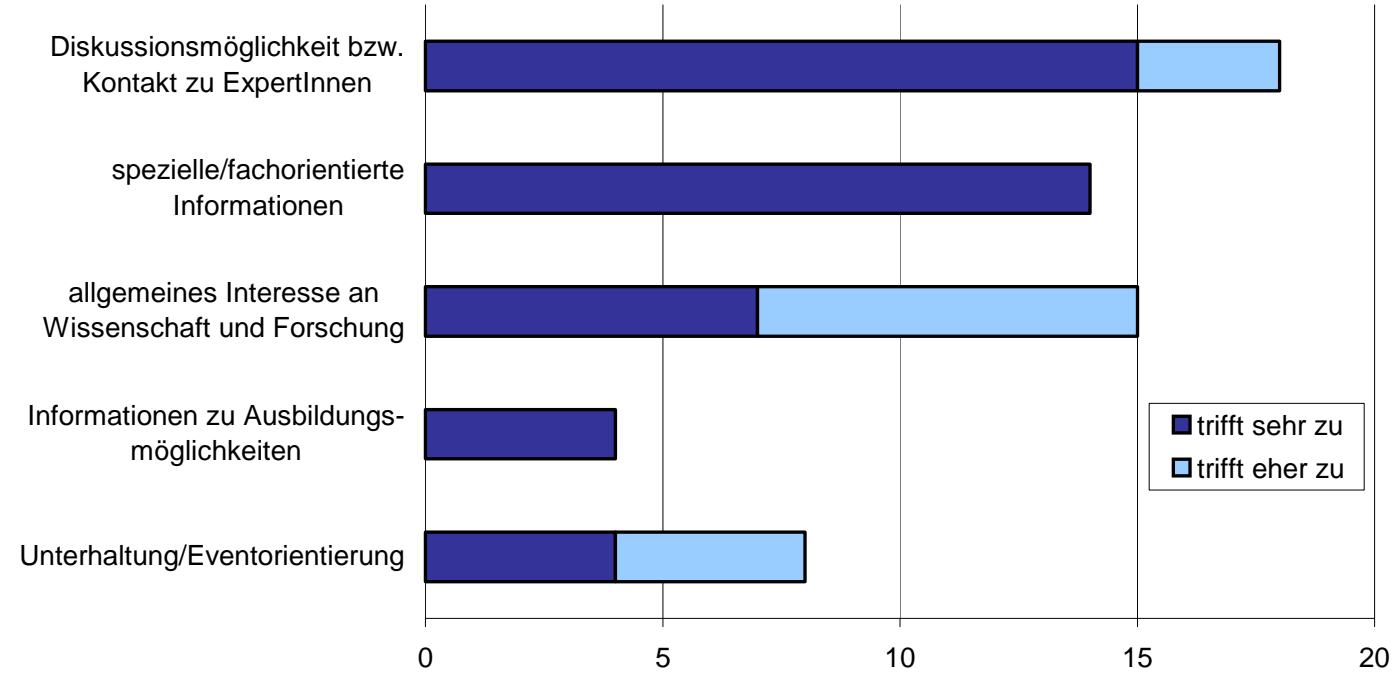

Anmerkungen:

$\mathrm{N}=16-19$

Anzahl der Antworten der Projektträger von zum Zeitpunkt der Befragung bereits stattgefundenen Projektaktivitäten; „trifft sehr zu“ bzw. „trifft eher zu“ auf die Frage: „Mit welchen Erwartungen sind aus Ihrer Sicht die Besucherlnnen bzw. Teilnehmerlnnen zu Ihren Projektaktivitäten gekommen?"

Quelle: KMU FORSCHUNG AUSTRIA, Online-Befragung Frühjahr 2006

Als primäre Effekte durch Projektaktivitäten erwarten sich die Projektträger/innen, wie aus Grafik 37 ersichtlich, einen Abbau von Hemmschwellen gegenüber Wissenschaft, Forschung und Technik und die Vermittlung des Nutzens der Forschung.

Jeweils 18 Projektträger/innen sehen die Forcierung der Berichterstattung über Forschung und Innovation und die Weckung von Interesse bzw. Neugier an Innovationsaktivitäten bei Jugendlichen als denkbare Effekte der Projektaktivitäten. Immerhin die Hälfte der Projektträger/innen gibt an, sich eine Weckung des Interesses/der Neugier an Innovationsaktivitäten bei der breiteren Öffentlichkeit zu erwarten, etwas weniger als ein Drittel können sich als Effekt die Weckung von Interesse/Neugier an Innovationsaktivitäten speziell bei Frauen vorstellen.

Die Förderung direkter Begegnungen zwischen Forscher/innen und Öffentlichkeit erwarten sich 16 Projektträger/innen als Wirkung ihrer Projektaktivitäten; 13 können sich eine Verbesserung der Kommunikationskompetenzen von FTI-Institutionen zur Öffentlichkeit als Nachwirkung vorstellen. Die Demonstration und Diskussion von Methoden und Vorgangsweisen der Forschung wird von 10 Projektträger/innen als möglicher Effekt der Projektaktivitäten gesehen. 
Grafik 37

Erwartete Effekte durch Projektaktivitäten im Rahmen der Kampagne - die Sicht der Projektträger/innen

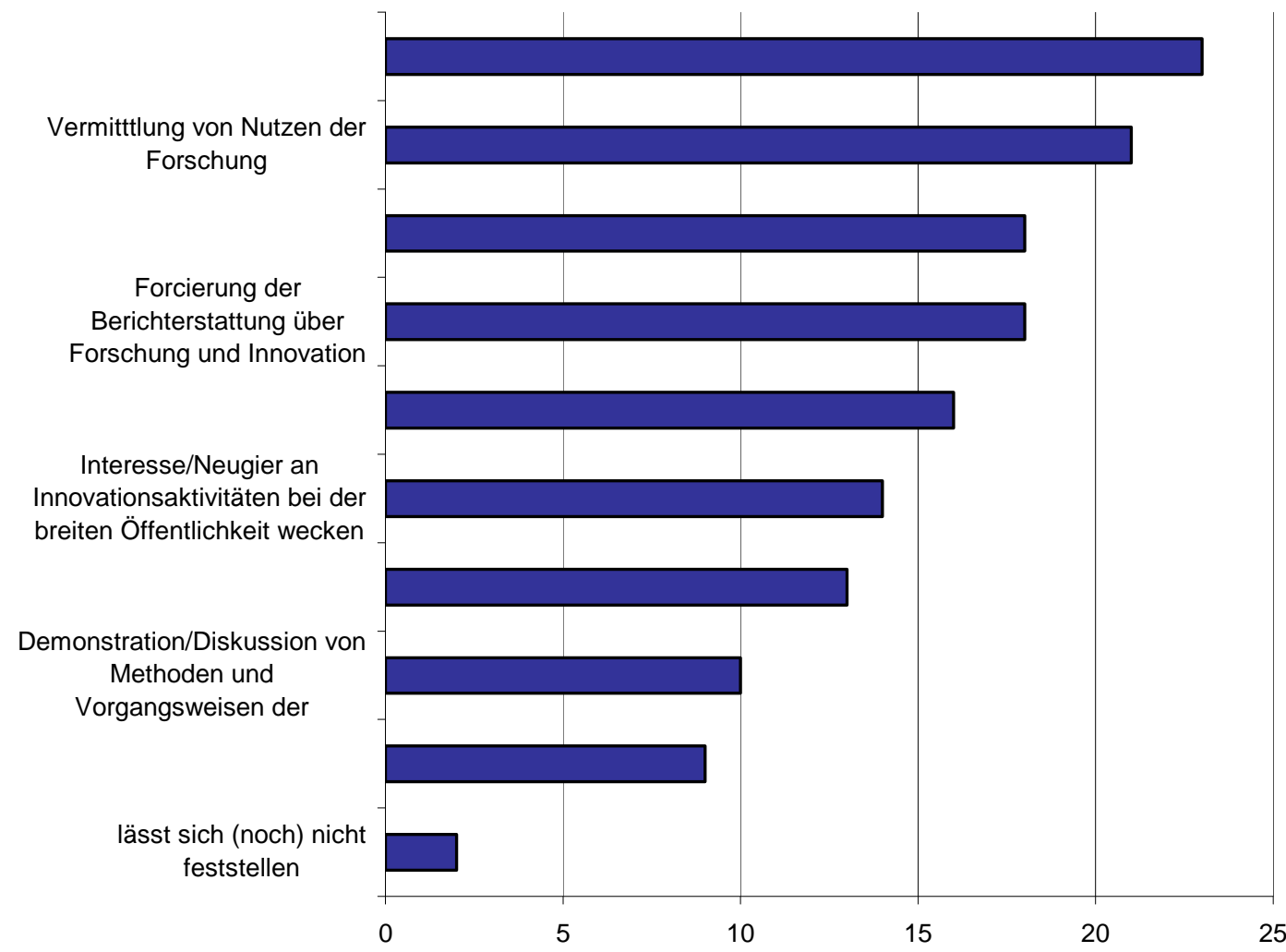

Anmerkungen:

$\mathrm{N}=28$, Mehrfachantworten möglich

Anzahl der Nennungen auf die Frage: „Welche Effekte erwarten Sie sich durch Ihre Aktivitäten im Rahmen der Kampagne innovatives-oesterreich.at?"

Quelle: KMU FORSCHUNG AUSTRIA, Online-Befragung Frühjahr 2006 


\section{Literatur}

Bauer, Martin W., Allum, Nick, Miller, Steve (2007): What can we learn from 25 years of PUS survey research? Liberating and expanding the agenda. Public Understanding of Science, Vol.16, 79-95

Besson, Nanette Aimée (2004): Strategische PR-Evaluation. Erfassung, Bewertung und Kontrolle von Öffentlichkeitsarbeit. 2.Aufl., Wiesbaden

Burns, T.W., O'Connor, D.J., Stocklmayer, S.M. (2003): Science Communication: a contemporary definition. In: Public Understanding of Science, Vol.12, 183-202

Eurobarometer (2005): Eurobarometer-Doppelerhebung „Bürger, Wissenschaft und Technologie“. FTEinfo - Magazin über europäische Forschung, Sonderausgabe November 2005

Felt, Ulrike (2000): Why Should the Public „Understand“ science? A Historical Perspective on Aspects of the Public Understanding of Science. In: M. Dierkes, C. von Grote (eds): Between Understanding and Trust: The Public, Science and Technology. Berkshire u.a., 7-38

Felt, Ulrike, gem. mit Müller, Annina und Schober, Sophie (2001): Evaluierung der Science Week@Austria. Analyse eines Experiments der Wissenschaftskommunikation im österreichischen Kontext. Im Auftrag des bm:bwk. Wien

Hampel, Jürgen, Renn, Ortwin (Hg.) (1999): Gentechnik in der Öffentlichkeit. Frankfurt a.M.

Leggewie, Claus (2006): Kampagnenpolitik. Eine nicht ganz neue Form politischer Mobilisierung. In: Ulrike Röttger (Hg.): PR-Kampagnen. Über die Inszenierung von Öffentlichkeit. 3. Aufl. Wiesbaden, 105-122

Miller, Steve (2001): Public understanding of science at the crossroads. Public Understanding of Science, Vol. 10, 115-120

Röttger, Ulrike (1998): Kampagnen. In: O. Jarren, U. Sarcinelli, U. Saxer (Hg.): Politische Kommunikation in der demokratischen Gesellschaft. Ein Handbuch mit Lexikonteil. Opladen, Wiesbaden 1998, 667

Röttger, Ulrike (Hg.) (2006): PR-Kampagnen. Über die Inszenierung von Öffentlichkeit. 3.Aufl. Wiesbaden

Schweiger, Günther et al. (2003): Wirkungsmessung einer Kampagne zur Verbesserung der Einstellungen der Österreicher gegenüber Ausgaben für Forschung und Entwicklung. www.innovatives-oesterreich.at. WU Wien. Wien

Weingart, Peter (2004): Welche Öffentlichkeit hat die Wissenschaft? In: Indre Zetsche (Hg): Wissenschaftskommunikation. Streifzug durch ein „neues“ Feld. Bonn, 15-21

Wynne, Brian (1992): Misunderstood misunderstanding: Social identities and public uptake of science. Public Understanding of Science, Vol.1, 281-304

Zetsche, Indre ( $\mathrm{Hg}$ ) (2004): Wissenschaftskommunikation. Streifzug durch ein „neues“ Feld. Bonn 



\section{Anhang I: Projekte der Kampagne innovatives-oesterreich.at}

\begin{tabular}{l|l}
\hline Projekttitel & Age of Simulation - Forschen und Lernen im 21. Jahrhundert \\
\hline $\begin{array}{l}\text { Projektträger } \\
\text { Projekttyp }\end{array}$ & $\begin{array}{l}\text { AEC ARS Ars Electronica Center Linz } \\
\text { Kurzbeschreibung des Projekts }\end{array}$ \\
$\begin{array}{l}\text { Ausstllung, Konferenz, Workshop } \\
\text { Ausgehend von Simulationen wurden in einer internationalen, trans- } \\
\text { disziplinären Konferenz und Ausstellung, das Spektrum der An- } \\
\text { wendungen von Simulationen dargestellt und die gesamtgesellschaft- } \\
\text { liche Relevanz von Simulation und ihre Grundlagen einer breiteren } \\
\text { Öffentlichkeit kommuniziert. } \\
\text { Jugendliche, Kinder, Frauen, Unternehmer/innen, } \\
\text { Wissenschaftler/innen, Studierende } \\
\text { K.A. }\end{array}$ \\
Besucher/Teilnehmerzahlen \\
Laufzeit
\end{tabular}

\begin{tabular}{l|l}
\hline Projekttitel & Applied Research in a box \\
\hline Projektträger & communication matters \\
Projekttyp & Web-Katalog \\
Kurzbeschreibung des Projekts & $\begin{array}{l}\text { Mit www.appliedresearch.at ist ein Katalog zu außeruniversitären } \\
\text { F\&E-Einrichtungen in Österreich, Dachorganisationen, Förderein- } \\
\text { richtungen sowie zu Programmen der Ministerien geschaffen worden. } \\
\text { www.appliedresearch.at soll einen Überblick über den Forschungs- } \\
\text { und Entwicklungsstandort Österreich geben und bei der Suche nach } \\
\text { Forschungspartnern und forschungsrelevanten Institutionen unter- } \\
\text { stützen. }\end{array}$ \\
$\begin{array}{l}\text { Zielgruppen } \\
\text { Besucher/Teilnehmerzahlen } \\
\text { Laufzeit }\end{array}$ & $\begin{array}{l}\text { krauen, Unternehmer/innen, Wissenschaftler/innen, Studierende } \\
\text { k.A. }\end{array}$ \\
\hline
\end{tabular}

\begin{tabular}{l|l}
\hline Projekttitel & Arbeitsplätze durch Innovation \\
\hline $\begin{array}{l}\text { Projektträger } \\
\text { Projekttyp }\end{array}$ & Arbeiterkammer Wien \\
Kurzbeschreibung des Projekts & $\begin{array}{l}\text { Wettbewerb } \\
\text { Firmen, die im Rahmen ihrer Forschungs- und Entwicklungsarbeit Ar- } \\
\text { beitsplätze schaffen und sichern konnten, wurden von BAK und FFG } \\
\text { ausgezeichnet. Kriterien für die Auszeichnung waren die Steigerung } \\
\text { der Mitarbeiterzahlen, aber auch die Arbeitsplatzqualität, Frauen- } \\
\text { förderungsmaßnahmen oder das Engagement bei der Aus- und } \\
\text { Weiterbildung der Mitarbeiter. Insgesamt gab es zehn Veran- } \\
\text { staltungen: acht regionale Landessiegerehrungen vor Ort, eine }\end{array}$ \\
$\begin{array}{l}\text { Jurysitzung zur Wahl der Bundessieger aus dem Kreis der Landes- } \\
\text { sieger in Wien und eine Bundessiegerveranstaltung in Wien. }\end{array}$ \\
$\begin{array}{l}\text { Zntelgrnehmer/innen } \\
\text { Besucher/Teilnehmerzahlen } \\
\text { Laufzeit }\end{array}$ & $\begin{array}{l}110 \text { (Bundessiegerehrung) } \\
01 / 2005-05 / 2006\end{array}$ \\
\hline
\end{tabular}




\begin{tabular}{l|l}
\hline Projekttitel & $\begin{array}{l}\text { Dachkampagne und Webplattform von } \\
\text { innovatives-oesterreich.at }\end{array}$ \\
\hline $\begin{array}{l}\text { Projektträger } \\
\text { Projekttyp } \\
\text { Kurzbeschreibung des Projekts }\end{array}$ & $\begin{array}{l}\text { Trimedia Communications Austria; uma information technology } \\
\text { Dachkampagne } \\
\text { Mit der Umsetzung der Kampagne und der Webplattform waren } \\
\text { Trimedia und uma als Leadagenturen beauftragt. Trimedia hat die } \\
\text { Dachmarke, klassische Werbung, Medienkooperationen und Medien- } \\
\text { auftritte verantwortet, die Projektträger auf Wunsch bei ihrer Medien- } \\
\text { arbeit unterstützt und Vernetzungstreffen aller Projektträger (Kick-off- } \\
\text { und Meet-up-Treffen) organisiert. Von der zweiten Leadagentur uma } \\
\text { stammte das Konzept der Fragenkampagne, die v.a. über die Website } \\
\text { umgesetzt wurde, das Frageteam für Veranstaltungen sowie Ge- } \\
\text { staltung und Content Management der Website. }\end{array}$ \\
$\begin{array}{l}\text { Zielgruppen } \\
\text { Besucher/Teilnehmerzahlen } \\
\text { Laufzeit }\end{array}$ & \begin{tabular}{l} 
ab/2005-12/2006 \\
\hline
\end{tabular}
\end{tabular}

\begin{tabular}{|c|c|}
\hline Projekttitel & Forschung für Uns \\
\hline Projektträger & PR\&D Public Relations for Research and Development \\
\hline Projekttyp & Diskussionsveranstaltungen \\
\hline Kurzbeschreibung des Projekts & $\begin{array}{l}\text { Forschung für Uns stand für Aktivitäten am Campus Vienna Biocenter } \\
\text { mit der involvierten Öffentlichkeit. Dabei stand der persönliche Nutzen } \\
\text { der Life Science-Forschung in Österreich - und damit von Bio- und } \\
\text { Gentechnologie allgemein - für individuelle Bürger/innen im Mittel- } \\
\text { punkt. Forschung für Uns umfasste folgende Maßnahmen: } \\
\text { - Medi Talks: Back-to-Back-Seminare von Wissenschaftler/innen- } \\
\text { und Mediziner/innen unter Teilnahme von Patient/innen und } \\
\text { Selbsthilfegruppen } \\
\text { Busi Talks: Internationale Seminare zu wirtschaftlichen Aspekten } \\
\text { der Life Science unter Teilnahme von Entscheidungsträger/innen, } \\
\text { Patient/innen und Selbsthilfegruppen } \\
\text { Sci News: Regelmäßige und kontinuierliche Medienbetreuung } \\
\text { vom Campus die sich inhaltlich auch an Entscheidungs- } \\
\text { träger/innen, Patient/innen und Selbsthilfegruppen wendet }\end{array}$ \\
\hline Zielgruppen & Jugendliche, Kinder, Frauen, Wissenschafter/innen, Studierende \\
\hline Besucher/Teilnehmerzahlen & 250 \\
\hline Laufzeit & 08/2005-12/2006 \\
\hline
\end{tabular}




\begin{tabular}{|c|c|}
\hline Projekttitel & Forschung mit Fun \\
\hline Projektträger & PR\&D Public Relations for Research and Development \\
\hline Projekttyp & Interaktive Veranstaltungen \\
\hline Kurzbeschreibung des Projekts & $\begin{array}{l}\text { Forschung mit Fun positionierte Life Science in der jugendlichen und } \\
\text { gering involvierten Öffentlichkeit. Wissenschaft wurde über die } \\
\text { emotionale Ebene mit Freizeitgestaltung verbunden: } \\
\text { - } \quad \text { Cine Talks: Events, bei denen ein auf wissenschaftlichen Inhalten } \\
\text { basierender Spielfilm gezeigt wurde. Eine lockere Diskussion mit } \\
\text { Expert/innen zu den molekularbiologischen Inhalten, deren Um- } \\
\text { setzbarkeit und Realitätsgehalt folgte im Anschluss. } \\
\text { - Sci ArtJunior: Kindergartengruppen erhielten Anregung und Mög- } \\
\text { lichkeiten zum Basteln durch Demonstrationen des Forschungs- } \\
\text { betriebes und Bereitstellung authentischer, ungefährlicher Ma- } \\
\text { terialien. } \\
\text { - Sci ArtSenior: Junge Künstler/innen erhielten "Inspirationen" } \\
\text { durch ein persönliches Gespräch über experimentelle Arbeiten. In } \\
\text { Reaktion darauf führten sie ihre künstlerischen Semesterarbeiten } \\
\text { aus. } \\
\text { Vernissage: Die "Kunstwerke" der beiden Maßnahmen Sci Art } \\
\text { wurden gemeinsam ausgestellt. Eine Einführung in das inspi- } \\
\text { rierende Experiment und eine "Ausstellung" der zur Verfügung } \\
\text { gestellten Materialien begleiteten die Vernissage. }\end{array}$ \\
\hline Zielgruppen & Jugendliche, Kinder, Frauen, Wissenschafter/innen, Studierende \\
\hline Besucher/Teilnehmerzahlen & 466 \\
\hline Laufzeit & $10 / 2005-12 / 2006$ \\
\hline
\end{tabular}

\begin{tabular}{l|l}
\hline Projekttitel & Forschungstage 2006 \\
\hline Projektträger & BMVIT \\
Projekttyp & Diverse Veranstaltungen \\
Kurzbeschreibung des Projekts & $\begin{array}{l}\text { Die Forschungstage 2006 umfassten unterschiedliche Veranstal- } \\
\text { tungen: vom Radioquiz bis zur feierlichen Eröffnung waren die For- } \\
\text { schungstage ein Angebot für die wachsende "scientific community" } \\
\text { genauso wie für die breite Öffentlichkeit. }\end{array}$ \\
Zielgruppen & $\begin{array}{l}\text { Jugendliche, Frauen, Unternehmer/innen, Wissenschafter/innen, } \\
\text { Studierende } \\
\text { Besucher/Teilnehmerzahlen } \\
\text { Laufzeit }\end{array}$ \\
\hline
\end{tabular}




\begin{tabular}{l|l}
\hline Projekttitel & Fotowettbewerb - Innovative Motive \\
\hline Projektträger & doppio espresso \\
Kurzbeschreibung des Projekts & Wettbewerb \\
Mit Unterstützung der Bundesinnung der Fotografen wurden alle in \\
Österreich professionell arbeitenden Fotograf/innen eingeladen, in 45 \\
Einrichtungen zu fotografieren. 59 Fotograf/innen nahmen an dem \\
Wettbewerb teil. Die Verleihung der Preise in vier thematischen, zwei \\
Herkunftskategorien sowie für den Gesamtsieger fand am 1. Februar \\
2006 im Museum der modernen Kunst Stiftung Ludwig in Wien vor \\
rd. 250 Besucher/innen statt. Die Preisverleihung war Startschuss für \\
die Wanderausstellung „Innovative Motive“. Siegerfotos und die \\
besten Motive wurden in von der Künstlergruppe „walking chair“ \\
gestalteten hintergrundbeleuchteten Schaukästen an insgesamt elf \\
Orten in Österreich bei 12 verschiedenen Veranstaltungen gezeigt. \\
Der Aufbau eines Bildarchivs gemeinsam mit der Partnerbildagentur \\
Buenos Dias zur Nutzung der Fotos für die Kommunikations- und \\
Verlagsbranche, sowie die Gestaltung des Bildbandes „Exploration F \\
- Österreichs Forschung im Fokus“ im Verlag Christian Brandstätter - \\
Gastgeschenk des BMVIT bei den vom Ministerium organisierten \\
Konferenzen während der EU-Ratspräsidentschaft Österreichs - \\
bildeten zwei weitere Schienen der Verwertung des im Wettbewerb \\
gewonnenen Fotomaterials. \\
Unternehmer/innen \\
Zielgruppen \\
Besucher/Teilnehmerzahlen \\
$\begin{array}{l}\text { Laufzeit } \\
\text { 250 (mind. 2.500 inkl. weiterer Ausstellungen der Bilder) } \\
11 / 2005-02 / 2006\end{array}$ \\
\hline
\end{tabular}

\begin{tabular}{l|l}
\hline Projekttitel & Frauen und Technik \\
\hline $\begin{array}{l}\text { Projektträger } \\
\text { Kurzbeschreibung des Projekts }\end{array}$ & $\begin{array}{l}\text { IV Salzburg } \\
\text { Workshops, Beratung } \\
\text { Ziel der Veranstaltungen in Kooperation mit Mädchen-/Frauen- } \\
\text { organisationen war es, mittels Beratungs-, Informations- und } \\
\text { Offentlichkeitsmaßnahmen und Aktionen mit Betrieben und Schulen } \\
\text { den Zugang zur Technik fördern und unterstützen: } \\
\text { Workshops zum Thema "Innovationen" und "Kreativität" an Salz- } \\
\text { burger Schulen. Als Trainer/innen wurden einerseits Mitar- } \\
\text { beiter/innen aus Betrieben (idealerweise Frauen in technischen } \\
\text { Berufen), andererseits Frauen aus einem der genannten Vereine } \\
\text { angesprochen. } \\
\text { Berufsinformationsmesse: Präsentation und Beratung }\end{array}$ \\
$\begin{array}{l}\text { Zielgruppen } \\
\text { Besucher/Teilnehmerzahlen } \\
\text { Laufzeit }\end{array}$ & $\begin{array}{l}\text { Frauen } \\
\text { k.A. } \\
\text { k.A. }\end{array}$ \\
\hline
\end{tabular}




\begin{tabular}{l|l}
\hline Projekttitel & Gender\&Science \\
\hline Projektträger & Science Communications \\
Projekttyp & Netzwerkevent, Symposium \\
Kurzbeschreibung des Projekts & $\begin{array}{l}\text { Im Vordergrund der Veranstaltungen stand die Vernetzung von Nach- } \\
\text { wuchswissenschafter/innen. } \\
\text { Wielgruppen }\end{array}$ \\
Besucher/Teilnehmerzahlen & 100 \\
Laufzeit & $02 / 2005-03 / 2005$ \\
\hline
\end{tabular}

\begin{tabular}{l|l}
\hline Projekttitel & Gödels Jahrhundert \\
\hline Projektträger & Österreichische Mathematische Gesellschaft \\
Projekttyp & Ausstellung \\
Kurzbeschreibung des Projekts & $\begin{array}{l}\text { Die Ausstellung präsentierte den weitgehend unbekannten Wiener } \\
\text { Mathematiker Kurt Gödel, der 2006 seinen 100. Geburtstag gefeiert } \\
\text { hätte, einer größeren Öffentlichkeit }\end{array}$ \\
Zielgruppen & Frauen, Unternehmer/innen, Wissenschafter/innen, Studierende \\
Besucher/Teilnehmerzahlen & 3.500 \\
Laufzeit & $04 / 2006-08 / 2006$ \\
\hline
\end{tabular}

\begin{tabular}{l|l}
\hline Projekttitel & High Tech - Low Quality \\
\hline Projektträger & $\begin{array}{l}\text { Science Communications } \\
\text { Projekttyp }\end{array}$ \\
Kurzbeschreibung des Projekts & $\begin{array}{l}\text { Diskussionsforum } \\
\text { Das Projekt hatte zum Ziel, einen nicht nur funktionalen Qualitäts- } \\
\text { begriff zu diskutieren. Die Veranstaltungsreihe war als Diskussions- } \\
\text { forum für ein möglichst breites Publikum konzipiert. }\end{array}$ \\
Zielgruppen & $\begin{array}{l}\text { Unternehmer/innen, Forscher/innen und Entwickler/innen, Studieren- } \\
\text { de, Lehrende und alle an Technik- und Wissenschaft Inter- }\end{array}$ \\
essent/innen \\
Besucher/Teilnehmerzahlen \\
Laufzeit
\end{tabular}




\begin{tabular}{l|l}
\hline Projekttitel & ideenreich.at \\
\hline Projektträger & $\begin{array}{l}\text { Dr. Hochegger Kommunikationsberatung } \\
\text { Projekttyp } \\
\text { Kurzbeschreibung des Projekts } \\
\text { Diese Innovationsplattform bot kreativen Bürger/innen, innovativen } \\
\text { Unternehmen, Lieferanten und Kunden die Möglichkeit, sich virtuell } \\
\text { am Innovationsprozess zu beteiligen. Unter www.ideenreich.at wur- } \\
\text { den 2005 innerhalb von zwei Monaten rd. 780 Ideen eingereicht. } \\
\text { Gemeinsam mit den Ideengebern wurde die aktive Vermarktung von } \\
20 \text { erfolgversprechenden Ideen gestartet. Mit www.ideenreich.at hat } \\
\text { das BMVIT in Österreich erstmalig eine permanente Ideenplattform } \\
\text { geschaffen. Dieser Ideenpool unterscheidet sich von anderen Ideen- } \\
\text { pools nicht nur durch die thematische Breite, sondern auch dadurch, } \\
\text { dass alle Bevölkerungsgruppen eingeladen sind sich am Innovations- } \\
\text { prozess zu beteiligen. } \\
\text { Jugendliche, Kinder, Frauen, Unternehmer/innen, } \\
\text { schafter/innen, Studierende } \\
780 \quad \text { Wissen- } \\
\text { Zielgruppen }\end{array}$ \\
$\begin{array}{l}\text { Besucher/Teilnehmerzahlen } \\
\text { Laufzeit }\end{array}$ & \begin{tabular}{l} 
2003-2005 \\
\hline
\end{tabular}
\end{tabular}

\begin{tabular}{l|l}
\hline Projekttitel & Innovation V \\
\hline Projektträger & IV-Vorarlberg \\
Projekttyp & Diskussionsveranstaltungsreihe \\
Kurzbeschreibung des Projekts & Die einzelnen Veranstaltungen zum Thema Innovation, gekoppelt mit \\
der jeweils sehr umfangreichen Vor- und Nachberichterstattung in den \\
Vorarlberger Nachrichten, trugen dazu bei, die Wichtigkeit von laufen- \\
der Innovation sowohl für den langfristigen Unternehmenserfolg als \\
auch für die zukünftige Wettbewerbsähigkeit der Region Vorarlberg \\
zu verdeutlichen und Unternehmen und Institutionen zu motivieren, \\
sich vermehrt mit Innovation und der Entwicklung von F\&E-Aktivitäten \\
auseinander zu setzen. \\
Ziele des Projekts: Abbau von Vorurteilen, Etablierung von Netz- \\
werken, ganzheitliche Kommunikation von Innovation, Herbeiführung \\
einer Bewusstseinsänderung \\
Unternehmer/innen, Wissenschafter/innen \\
Zielgruppen \\
Besucher/Teilnehmerzahlen \\
Laufzeit
\end{tabular}




\begin{tabular}{l|l}
\hline Projekttitel & Innovationsgespräche \\
\hline $\begin{array}{l}\text { Projektträger } \\
\text { Kurzbesttyp }\end{array}$ & IV-Steiermark / innoregio \\
& $\begin{array}{l}\text { Ziele der Steirischen Innovationsgespräche: die Analyse der steiri- } \\
\text { schen Innovationsaktivitäten, Einbindung der politischen Entschei- } \\
\text { dungsträger, Steigerung der public awareness für Innovation und } \\
\text { F\&E, Abstimmung von Strategien der Landes- und Bundespolitik, } \\
\text { Vergleich und Einbindung internationaler Benchmarkregionen und } \\
\text { Netzwerkaufbau im Bereich F\&E in der "EU-Zukunftsregion Südost". } \\
\text { Im Rahmen der Steirsichen Innovationsgespräche wurde das } \\
\text {,innobooklet styria“, der Informationsfolder ,Innovation in steirischen } \\
\text { Betrieben“ präsentiert. Das innobooklet dient als Informations- und } \\
\text { Bewusstseinsbildungsinstrument, um die steirischen Unternehmen } \\
\text { über alle Größen und Branchen hinweg, aber auch Meinungsbildner } \\
\text { und die Öffentlichkeit über die Bedeutung von Innovation für die } \\
\text { Steiermark noch stärker zu sensibilisieren und eine Verbreiterung der } \\
\text { Innovationsspitze zu forcieren. Das innobooklet wurde an alle Be- } \\
\text { triebe >10 Mitarbeiter in der Steiermark (ca. 3500 Betriebe) versandt, } \\
\text { mit der Zielsetzung noch nicht innovierende Betriebe zu ermutigen } \\
\text { und so eine langfristige Verbreiterung der steirischen Innovations- } \\
\text { spitze zu erwirken. } \\
\text { Unternehmer/innen, Wissenschafter/innen } \\
150\end{array}$ \\
$\begin{array}{l}\text { Zielgruppen } \\
\text { Lesucher/Teilnehmerzahlen } \\
\text { Laufzeit }\end{array}$ & \begin{tabular}{l} 
2005 \\
\hline
\end{tabular}
\end{tabular}

\begin{tabular}{|c|c|}
\hline Projekttitel & Innovationstag \\
\hline Projektträger & IV-Salzburg \\
\hline Projekttyp & Informations- und Diskussionsveranstaltung \\
\hline Kurzbeschreibung des Projekts & $\begin{array}{l}\text { Im Rahmen des Innovationstages präsentierten hochinnovative Unter- } \\
\text { nehmen, wie Innovation in der Praxis erfolgreich funktioniert. Zwi- } \\
\text { schen den Vorträgen bestand die Möglichkeit, mit Expert/innen Er- } \\
\text { fahrungen zum Thema Innovation auszutauschen. Im Rahmen des } \\
\text { Innovationstages erhalten Unternehmen die Möglichkeit, von den } \\
\text { Top-Managern erfolgreicher Unternehmen zu erfahren, was in der } \\
\text { betrieblichen Praxis tatsächlich entscheidend ist. }\end{array}$ \\
\hline Zielgruppen & Unternehmer/innen \\
\hline Besucher/Teilnehmerzahlen & k.A. \\
\hline Laufzeit & $11 / 2005$ \\
\hline
\end{tabular}

\begin{tabular}{l|l}
\hline Projekttitel & Innovations-Updates \\
\hline Projektträger & IV-Salzburg \\
Projekttyp & Veranstaltungsreihe \\
Kurzbeschreibung des Projekts & $\begin{array}{l}\text { Insgesamt fanden acht Vorträge und Workshops statt. Eingeladen } \\
\text { wurden Expert/innen aus den Bereichen F\&E und Innovations- } \\
\text { management in Salzburger Unternehmen. } \\
\text { Unternehmer/innen, Wissenschafter/innen }\end{array}$ \\
Zielgruppen & $\begin{array}{l}\text { k.A. } \\
\text { Besucher/Teilnehmerzahlen }\end{array}$ \\
\hline
\end{tabular}




\begin{tabular}{l|l}
\hline Projekttitel & innovatives-unternehmen \\
\hline $\begin{array}{l}\text { Projektträger } \\
\text { Projekttyp } \\
\text { Kurzbeschreibung des Projekts }\end{array}$ & $\begin{array}{l}\text { WIFI } \\
\text { Veranstaltungen, Plattform } \\
\text { Das ganze Jahr 2006 über fanden in allen Bundesländern Veran- } \\
\text { staltungsreihen statt, bei denen das Thema "innovatives-unter- } \\
\text { nehmen" aus neuen Blickwinkeln betrachtet wurden. Mit einer } \\
\text { Partner-Plattform von österreichischen Spitzenunternehmen war be- } \\
\text { absichtigt, gemeinsam Innovation bei österreichischen KMU zu } \\
\text { fördern und voranzutreiben, Beispiele erfolgreicher innovativer Unter- } \\
\text { nehmen aus Österreich aufzuzeigen und somit mehr Bewusstsein für } \\
\text { die Bedeutung von Innovation in den Betrieben zu schaffen. } \\
\text { Unternehmer/innen }\end{array}$ \\
$\begin{array}{l}\text { Zielgruppen } \\
\text { Besucher/Teilnehmerzahlen } \\
\text { Laufzeit }\end{array}$ & $\begin{array}{l}5.000 \\
01 / 2006-12 / 2006\end{array}$ \\
\hline
\end{tabular}

\begin{tabular}{l|l}
\hline Projekttitel & International Networking for Young Scientists \\
\hline Projektträger & British Council Austria \\
Projekttyp & Workshop \\
Kurzbeschreibung des Projekts & $\begin{array}{l}\text { Gegenstand des Projekts waren persönliche Zusammentreffen von } \\
\text { jungen Wissenschafter/innen und Forscher/innen aus unterschied- }\end{array}$ \\
$\begin{array}{l}\text { lichen Staaten zwecks des Austauschs von Ideen, Wissen und } \\
\text { Informationen sowie Netzwerkaufbau. } \\
\text { Zielgruppen } \\
\text { Besucher/Teilnehmerzahlen } \\
\text { Laufzeit }\end{array}$ & $\begin{array}{l}\text { k.A. } \\
02 / 2005\end{array}$ \\
\hline
\end{tabular}

\begin{tabular}{l|l}
\hline Projekttitel & iScan \\
\hline Projektträger & IV-Steiermark (innoregio) \\
Projekttyp & Web-Tool \\
Kurzbeschreibung des Projekts & $\begin{array}{l}\text { Der iScan ist ein Diagnoseinstrument im Internet, welches Unter- } \\
\text { nehmen eine erste Einschätzung ihrer Innovationskraft gibt. Sie } \\
\text { gehen durch sechs unterschiedliche "Räume" im "Haus der Inno- } \\
\text { vation" und erhalten ein Innovationsprofil ihres Unternehmens sowie } \\
\text { eine Benchmarkanalyse. } \\
\text { Unternehmer/innen }\end{array}$ \\
Zielgruppen & k.A. \\
Besucher/Teilnehmerzahlen & $10 / 2005$
\end{tabular}




\begin{tabular}{l|l}
\hline Projekttitel & Jobmotor Forschung \\
\hline Projektträger & $\begin{array}{l}\text { Gewerkschaft der Privatangestellten (GPA) } \\
\text { Projekttyp }\end{array}$ \\
Kurzbeschreibung des Projekts & $\begin{array}{l}\text { Mit der Konferenzreihe Jobmotor Forschung wurden betriebliche } \\
\text { Interessenvertretungen und Führungskräfte mit Expert/innen aus } \\
\text { Forschungseinrichtungen und Förderstrukturen zusammengeführt. } \\
\text { Zweistündige Konferenzen bildeten den Rahmen zum Wissensaus- } \\
\text { tausch und Netzwerken. }\end{array}$ \\
Zielgruppen & $\begin{array}{l}\text { Frauen, Unternehmer/innen, Wissenschaftler/innen, Studierende } \\
\text { Besucher/Teilnehmerzahlen } \\
\text { Laufzeit }\end{array}$ \\
\hline
\end{tabular}

\begin{tabular}{|c|c|}
\hline Projekttitel & Jugend Innovativ \\
\hline Projektträger & aws - Austria Wirtschaftsservice \\
\hline Projekttyp & Wettbewerb \\
\hline Kurzbeschreibung des Projekts & $\begin{array}{l}\text { Jugend Innovativ ist der Schulwettbewerb für innovative Ideen aus } \\
\text { den Bereichen Business, Design, Engineering und Science. Seit } 20 \\
\text { Jahren beweisen Österreichs Schülerinnen und Schüler, dass Inno- } \\
\text { vation nicht nur eine Sache der "Erwachsenen" ist, indem sie geniale } \\
\text { Ideen und Entwicklungen in Form von Projekten ausarbeiten und im } \\
\text { Rahmen von Jugend Innovativ der Öffentlichkeit präsentieren. }\end{array}$ \\
\hline Zielgruppen & Jugendliche \\
\hline Besucher/Teilnehmerzahlen & 1.069 \\
\hline Laufzeit & 2005,2006 \\
\hline
\end{tabular}

\begin{tabular}{l|l}
\hline Projekttitel & Junge Uni Innsbruck \\
\hline Projektträger & Universität Innsbruck \\
Projekttyp & Kinderuniversität \\
Kurzbeschreibung des Projekts & Ziel ist es, Kinder und Jugendliche für Wissenschaft und Forschung \\
& zu begeistern. \\
Zielgruppen & Kinder, Jugendliche \\
Besucher/Teilnehmerzahlen & 15.000 \\
Laufzeit & $2004-2006$ \\
\hline
\end{tabular}

\begin{tabular}{|c|c|}
\hline Projekttitel & Junge Uni Krems \\
\hline Projektträger & IMC FH Krems \\
\hline Projekttyp & Kinderuniversität \\
\hline Kurzbeschreibung des Projekts & $\begin{array}{l}\text { Unter dem Titel "Junge Uni der FH Krems" veranstaltete die IMC } \\
\text { Fachhochschule von 10. bis } 14 \text {. Juli } 2006 \text { eine Woche lang für } 11- \\
14 \text { Jährige richtigen Uni-Betrieb. }\end{array}$ \\
\hline Zielgruppen & Jugendliche, Kinder \\
\hline Besucher/Teilnehmerzahlen & 200 \\
\hline Laufzeit & 2006 \\
\hline
\end{tabular}




\begin{tabular}{l|l}
\hline Projekttitel & Kinderuni Steyr \\
\hline Projektträger & IFAU Institut für Angewandte Umweltbildung \\
Projekttyp & Kinderuniversität \\
Kurzbeschreibung des Projekts & $\begin{array}{l}\text { Die Kinderuni Steyr ermöglicht Kindern einen Blick in die Welt der } \\
\text { Forschung. Die Kinder sollen mit renommierten Wissenschaftler/innen } \\
\text { und Expert/innen aus der Praxis in Kontakt kommen, die ihr Leben } \\
\text { einem Fach, Thema oder Spezialaufgaben widmen. }\end{array}$ \\
Zielgruppen & $\begin{array}{l}\text { Jugendliche, Kinder } \\
\text { Besucher/Teilnehmerzahlen } \\
\text { Laufzeit }\end{array}$ \\
\hline
\end{tabular}

\begin{tabular}{l|l}
\hline Projekttitel & Kinderuni Wien \\
\hline $\begin{array}{l}\text { Projektträger } \\
\text { Projekttyp } \\
\text { Kurzbeschreibung des Projekts }\end{array}$ & $\begin{array}{l}\text { Universität Wien } \\
\text { Kinderuniversität } \\
\text { Im Rahmen des Projekts Kinderuni Wien, das vom Kinderbüro bereits } \\
\text { zum vierten Mal organisiert wurde, lernen Kinder die Universität } \\
\text { kennen und sind für zwei Wochen Studentin oder Student. Angeboten } \\
\text { werden Vorlesungsbesuche, Workshops und Ateliers an der Kinder- } \\
\text { uni-Kunst, der Kinderuni-Wissenschaft und der Kinderuni-Medizin, } \\
\text { inklusive Studienausweis, Studienbuch und Sponsion. } \\
\text { Jugendliche, Kinder } \\
\text { Zielgruppen } \\
\text { Besucher/Teilnehmerzahlen } \\
\text { Laufzeit }\end{array}$ \\
\hline
\end{tabular}

\begin{tabular}{l|l}
\hline Projekttitel & Kommunikationswissenschaftliche Tage \\
\hline $\begin{array}{l}\text { Projektträger } \\
\text { Projekttyp } \\
\text { Kurzbeschreibung des Projekts }\end{array}$ & $\begin{array}{l}\text { ITA - Institut für Technikfolgenabschätzung } \\
\text { Informations- und Diskussionsveranstaltung } \\
\text { An zwei Konferenztagen diskutierten internationale Expert/innen Kon- } \\
\text { zepte, Ausmaß, Strukturbedingungen und Veränderungen europäi- } \\
\text { scher Öffentlichkeit unter dem Einfluss des medialen und gesell- } \\
\text { schaftlichen Wandels in den Räumlichkeiten der Vertretung der } \\
\text { Europäischen Kommission in Österreich. Die Konferenzbeiträge boten } \\
\text { einen Überblick zum aktuellen Stand der transdisziplinären euro- } \\
\text { päischen Öffentlichkeitsforschung aus kommunikationswissenschaft- }\end{array}$ \\
licher, soziologischer, politikwissenschaftlicher und historischer \\
Perspektive. Ebenso wurde eine Podiumsdiskussion mit Journa-
\end{tabular}




\begin{tabular}{l|l}
\hline Projekttitel & Kreatives Kärten \\
\hline Projektträger & IV-Kärnten \\
Projekttyp & Wettbewerb \\
Kurzbeschreibung des Projekts & $\begin{array}{l}\text { Projektziel: Schulen für das Innovationsthema zu begeistern. Im } \\
\text { Rahmen dieses Wettbewerbs waren eigene Produkt- oder Dienst- } \\
\text { leistungsideen zu entwickeln sowie ein Vermarktungskonzept zu } \\
\text { entwerfen. }\end{array}$ \\
$\begin{array}{l}\text { Zielgruppen } \\
\text { Besucher/Teilnehmerzahlen } \\
\text { Laufzeit }\end{array}$ & $\begin{array}{l}\text { J.A. } \\
09 / 2005-06 / 2006\end{array}$ \\
\hline
\end{tabular}

\begin{tabular}{|c|c|}
\hline Projekttitel & Lange Nacht der Forschung 2005 \\
\hline Projektträger & Science Communications \\
\hline \multicolumn{2}{|l|}{ Projekttyp } \\
\hline Kurzbeschreibung des Projekts & $\begin{array}{l}\text { Von drei "Treffpunkten Forschung" im jeweiligen Stadtzentrum (Wien, } \\
\text { Linz, Innsbruck) führten } 13 \text { Busrouten zu "Stationen der Forschung". } \\
\text { Insgesamt } 150 \text { solcher Stationen zeigten unterschiedlichste Positio- } \\
\text { nen der zeitgenössischen Forschung aus vielen Disziplinen - wie } \\
\text { Architektur, IT, Medizin, Technik oder Umwelt - zum Thema Sicher- } \\
\text { heit. Sie zeigten, wo Forschung passiert, wie Forschungsfragen ent- } \\
\text { stehen und wie die Gesellschaft von aktuellen Forschungen beein- } \\
\text { flusst wird. Besucher/innen hatten dabei die Möglichkeit, an For- } \\
\text { schungsprozessen aktiv teil zu haben, Forscher/innen ganz persön- } \\
\text { lich zu begegnen und mit ihnen und anderen Interessierten aktuelle } \\
\text { Fragen und Entwicklungen in Forschung sowie Gesellschaft zu disku- } \\
\text { tieren. }\end{array}$ \\
\hline Zielgruppen & $\begin{array}{l}\text { Jugendliche, Kinder, Frauen, Unternehmer/innen, Wissen- } \\
\text { schafter/innen, Studierende }\end{array}$ \\
\hline Besucher/Teilnehmerzahlen & 48.000 \\
\hline Laufzeit & $10 / 2005$ \\
\hline
\end{tabular}

\begin{tabular}{l|l}
\hline Projekttitel & Language of Networks \\
\hline Projektträger & AEC ARS Electronica \\
Projekttyp & $\begin{array}{l}\text { Symposium und Ausstellung } \\
\text { Kurzbeschreibung des Projekts } \\
\text { Bei dem internationalen Symposium "Language of Networks" disku- } \\
\text { tierten Expert/innen aus sieben Ländern die Methode der "Sozialen }\end{array}$ \\
& $\begin{array}{l}\text { Netzwerkanalyse" und ihre vielfältigen Anwendungen. Eine Aus- } \\
\text { stellung zum selben Thema ermöglichte Einblicke in Möglichkeiten } \\
\text { der Netzwerkvisualisierung. }\end{array}$ \\
Zielgruppen & $\begin{array}{l}\text { Frauen, Unternehmer/innen, Wissenschafter/innen, Studierende } \\
\text { Besucher/Teilnehmerzahlen } \\
\text { Laufzeit }\end{array}$ \\
\hline
\end{tabular}




\begin{tabular}{l|l}
\hline Projekttitel & Lehre und Forschung \\
\hline Projektträger & Plansinn \\
Projekttyp & Workshops, Diskussionsveranstaltungen \\
Kurzbeschreibung des Projekts & $\begin{array}{l}\text { Ziel des Projekts war die Herstellung einer Annäherung zwischen } \\
\text { wissenschaftlichen Diskussionen und dem Alltag von jugendlichen } \\
\text { Berufsschüler/innen. In den zentralen sechs Projektworkshops ent- } \\
\text { wickelten Lehrlinge unterschiedlicher Branchen gemeinsam mit } \\
\text { Wissenschaftler/innen Produkte (Texte, Installationen, Präsentationen } \\
\text { etc.), die jeweils bei einem Event öffentlich präsentiert wurden. }\end{array}$ \\
Zielgruppen \\
Besucher/Teilnehmerzahlen \\
Laufzeit
\end{tabular}

\begin{tabular}{l|l}
\hline Projekttitel & Mapping Controversities \\
\hline Projektträger & ÖAW \\
Projekttyp & Diskussionsveranstaltung \\
Kurzbeschreibung des Projekts & $\begin{array}{l}\text { Im Rahmen der Veranstaltung diskutierten Wissenschafter/innen und } \\
\text { Künstler/innen aus Österreich und dem Ausland über alternative For- } \\
\text { men der Vermittlung wissenschaftlicher Ergebnisse und Forschungs- } \\
\text { prozesse. Das Publikum war zu einer visuellen Erkundung der } \\
\text { aktuellen Diskussion über gentechnisch modifizierte Nahrungsmittel } \\
\text { eingeladen. Zu Anwendung kamen dabei auch neue Techniken dyna- } \\
\text { mischer Kartographie. Darüber hinaus veranschaulichte eine spezielle } \\
\text { multimediale Installation den Besuchern die ethischen, sozialen und } \\
\text { politischen Facetten dieser Debatte. }\end{array}$ \\
Zielgruppen & $\begin{array}{l}\text { Jugendliche, Frauen, Unternehmer/innen, Wissenschafter/innen, } \\
\text { Studierende } \\
\text { Besucher/Teilnehmerzahlen } \\
\text { Laufzeit }\end{array}$ \\
\hline
\end{tabular}

\begin{tabular}{|c|c|}
\hline Projekttitel & Medienkooperation im Wettbewerb \\
\hline Projektträger & doppio espresso \\
\hline Projekttyp & Wettbewerb \\
\hline Kurzbeschreibung des Projekts & $\begin{array}{l}\text { Das Projekt zielte auf die Etablierung einer neuen, umfassenden } \\
\text { österreichischen Medienplattform für den Diskurs zu Forschung, } \\
\text { Technologie und Innovation ab. Insgesamt bewarben sich } 11 \text { Verlage; } \\
\text { den Zuschlag erhielt der Verlag LW-Media mit dem Format } \\
\text { „at.venture“. Das Magazin „at.venture“ ist umfassend in dem Sinn, als } \\
\text { es dem gesamten Innovationssystem und seinen Akteuren verpflichtet } \\
\text { ist: von der Grundlagenforschung bis zur Innovation auf dem Markt, } \\
\text { von den Hochschulen und den Institutionen der außeruniversitären } \\
\text { Forschung bis zu den Forschungs- und Entwicklungsabteilungen der } \\
\text { Unternehmen. Ein besonderer Fokus wird dabei auf die Verwertungs- } \\
\text { perspektive und die Umsetzung in Innovationen auf dem Markt gelegt. }\end{array}$ \\
\hline Zielgruppen & Frauen, Unternehmer/innen, Wissenschafter/innen, Studierende \\
\hline Besucher/Teilnehmerzahlen & 150 \\
\hline Laufzeit & $12 / 2005-09 / 2006$ \\
\hline
\end{tabular}




\begin{tabular}{l|l}
\hline Projekttitel & Meet the Media \\
\hline Projektträger & $\begin{array}{l}\text { communication matters } \\
\text { Diskussionsveranstaltungen } \\
\text { Kurzbeschreibung des Projekts } \\
\text { Öffentlichkeitsarbeiter/innen im Bereich FTE und Wissenschaft- } \\
\text { ler/innen kennen oftmals die Gesetzmäßigkeiten des Medienmarktes } \\
\text { nur unzureichend. Wissenschafts-Journalist/innen klagen ihrerseits } \\
\text { darüber, dass sie oft schlecht aufbereitete bis inhaltich unverständ- } \\
\text { liche Texte aus dem Bereich der Forschung und Technologieent- } \\
\text { wicklung bekommen. Im Abstand von zwei Monaten fanden insge- } \\
\text { samt fünf Veranstaltungen statt, die jeweils einem anderen Medientyp } \\
\text { gewidmet waren. Die vortragenden Journalist/innen führten in die } \\
\text { Besonderheiten ihres Mediums ein und erklärten die Kriterien, die } \\
\text { darüber entscheiden, ob Beiträge angenommen werden. } \\
\text { Unternehmer/innen, Wissenschafter/innen } \\
\text { Zielgruppen } \\
\text { Besucher/Teilnehmerzahlen } \\
\text { Laufzeit }\end{array}$ \\
\hline $\begin{array}{l}250 \\
11 / 2005-12 / 2006\end{array}$ \\
\hline
\end{tabular}

\begin{tabular}{|c|c|}
\hline Projekttitel & Open House der Forschung - Club Research \\
\hline Projektträger & doppio espresso \\
\hline Projekttyp & Diskussionsveranstaltungen \\
\hline \multirow[t]{3}{*}{ Kurzbeschreibung des Projekts } & $\begin{array}{l}\text { Mit dem Projekt wurde die Etablierung des Hauses der Forschung } \\
\text { zum Anlass genommen, Chance und Verpflichtung, eine gemein- } \\
\text { same, übergreifende Kommunikation für das gesamte Spektrum der } \\
\text { Forschung zu entwickeln, die alte, nicht mehr den Anforderungen } \\
\text { moderner Innovationssysteme entsprechenden Trennungen über- } \\
\text { windet. Insbesondere ging es um die Überwindung alter Dichotomien } \\
\text { von Grundlagen- und angewandter Forschung, die sich auch in dicho- } \\
\text { tomisch organisierte Kommunikationsstrukturen - bis hinein in die } \\
\text { Redaktionen der Medien selbst - überträgt. }\end{array}$ \\
\hline & $\begin{array}{l}\text { Das Veranstaltungsmodul „Club Research“ nutzte öffentliche } \\
\text { Räume für eine offene Diskursplattform, die aktuelle und } \\
\text { grundsätzliche Fragestellungen und Themen von Forschung } \\
\text { und Innovation aufgreift - unter Berücksichtigung eines um- } \\
\text { fassenden Begriffs von Forschung und Innovation, eines multi- } \\
\text { disziplinären Ansatzes und im Besonderen eines Austausches } \\
\text { der Forschungscommunity mit den Vertreter/innen der Medien. }\end{array}$ \\
\hline & $\begin{array}{l}\text { - } \quad \text { Vas Veranstaltungsmodul "Open House: Gesellschaft fragt, } \\
\text { Forschung antwortet" inszenierte Öffentlichkeit im Haus der } \\
\text { Forschung selbst. Vermittelt durch die Erhebung von Markt- } \\
\text { forschungsinstituten wurden Ansprüche, Erwartungshaltungen } \\
\text { und/oder Fragen der Gesellschaft an die Forschung formuliert } \\
\text { und in das Forum im Haus eingebracht, wo sie den Input und } \\
\text { Strukturrahmen für moderierte Diskussionen bildeten. }\end{array}$ \\
\hline Zielgruppen & Frauen, Unternehmer/innen, Wissenschafter/innen, Studierende \\
\hline Besucher/Teilnehmerzahlen & 180 \\
\hline Laufzeit & 02/2006-12/2006 \\
\hline
\end{tabular}




\begin{tabular}{|c|c|}
\hline Projekttitel & Pressereise Schweden \\
\hline Projektträger & communication matters \\
\hline Projekttyp & Exkursion \\
\hline Kurzbeschreibung des Projekts & $\begin{array}{l}\text { Schweden gilt in vielerlei Hinsicht als positives Beispiel für gelungene } \\
\text { FTI-Politik. Das gilt für das Schulsystem, bei dem Schweden in den } \\
\text { Naturwissenschaften einen Spitzenplatz belegt, weiters für das } \\
\text { universitäre und außeruniversitäre System und auch für die For- } \\
\text { schung der Unternehmen. Schweden gilt auch als Vorbild in Fragen } \\
\text { des Gender-Mainstreaming. Eine organisierte Reise konnte öster- } \\
\text { reichischen Journalist/innen und Expert/innen aus dem FTI-System } \\
\text { Aufschluss darüber geben, was aus dem System des nordischen } \\
\text { Landes als Benchmark für Österreich dienen kann. }\end{array}$ \\
\hline Zielgruppen & Unternehmer/innen, Wissenschafter/innen \\
\hline Besucher/Teilnehmerzahlen & k.A. \\
\hline Laufzeit & 09/2005-06/2006 \\
\hline
\end{tabular}

\begin{tabular}{l|l}
\hline Projekttitel & Public Understanding of Life Sciences \\
\hline $\begin{array}{l}\text { Projektträger } \\
\text { Projekttyp } \\
\text { Kurzbeschreibung des Projekts }\end{array}$ & $\begin{array}{l}\text { dialog gentechnik } \\
\text { diverse Veranstaltungen } \\
\text { Die Basisaktivitäten des Vereins dialog gentechnik umfassen den } \\
\text { Betrieb einer Informationsstelle für Anfragen zum Thema Gentechnik, } \\
\text { laufende fachliche Unterstützung von Journalist/innen und Wissen- } \\
\text { schafter/innen bei ihrer Öffentlichkeitsarbeit sowie verständliche Auf- } \\
\text { bereitung biowissenschaftlicher Themen. Die Palette erstreckt sich } \\
\text { von Artikeln auf der dialog gentechnik-Homepage über aktuelle Inter- } \\
\text { views mit Wissenschafter/innen bis zur Beteiligung an Projek- } \\
\text { ten/Veranstaltungen im Bereich Wissenschaftskommunikation. }\end{array}$ \\
$\begin{array}{l}\text { Zielgruppen } \\
\text { Jugendliche, Kinder, Frauen, Unternehmer/innen, Wissen- } \\
\text { schafter/innen, Studierende } \\
\text { k.A. } \\
\text { Laufzeit }\end{array}$ & 2005,2006
\end{tabular}

\begin{tabular}{|c|c|}
\hline Projekttitel & Quintessenz \\
\hline Projektträger & Z.ONE media \\
\hline Projekttyp & Produktion von Videodokumentationen \\
\hline Kurzbeschreibung des Projekts & $\begin{array}{l}\text { Im Rahmen des Projekts wurden fünf Videodokumentationen über die } \\
\text { Forschungstätigkeit an österreichischen Museen produziert. Die } \\
\text { grundlegende Forschungsfrage, die dabei geklärt werden sollte war: } \\
\text { Welchen Objektbegriff und welchen Umgang mit Objekten muss ein } \\
\text { Museum entwickeln, um spezifische Themen darstellen und vermitteln } \\
\text { zu können? }\end{array}$ \\
\hline Zielgruppen & $\begin{array}{l}\text { Jugendliche, Kinder, Frauen, Unternehmer/innen, Wissen- } \\
\text { schafter/innen, Studierende }\end{array}$ \\
\hline Besucher/Teilnehmerzahlen & k.A. \\
\hline Laufzeit & 08/2005-10/2006 \\
\hline
\end{tabular}




\begin{tabular}{l|l}
\hline Projekttitel & Schlaufuchsakademie Ennstal \\
\hline Projektträger & IFAU Institut für Angewandte Umweltforschung \\
Projekttyp & Kinderakademie \\
Kurzbeschreibung des Projekts & Sommerakademie der Kinderuni Steyr \\
Zielgruppen & Kinder, Jugendliche \\
Besucher/Teilnehmerzahlen & k.A. \\
Laufzeit & $2004-2006$ \\
\hline
\end{tabular}

\begin{tabular}{|c|c|}
\hline Projekttitel & SciMedia - Universitätslehrgang \\
\hline Projektträger & IFF - Fakultät für Interdisziplinäre Forschung und Fortbildung \\
\hline Projekttyp & Qualifizierungsmaßnahme \\
\hline Kurzbeschreibung des Projekts & $\begin{array}{l}\text { Ziel des Lehrgangs ist eine nachhaltige Stärkung der Kompetenz im } \\
\text { Bereich Wissenschaftsvermittlung in Österreich. Durch Ausbildung } \\
\text { von Personen soll das Verhältnis zwischen Wissenschaft und Öffent- } \\
\text { lichkeit, zwischen den Universitäten und den Medien verbessert und } \\
\text { weiter professionalisiert werden - zum Nutzen der Gesellschaft wie } \\
\text { auch der Wissenschaft. Den Teilnehmer/innen des Lehrgangs wurden } \\
\text { einerseits die wichtigsten Fähigkeiten für journalistische Wissen- } \\
\text { schaftsvermittlung bzw. Wissenschaftskommunikation im PR-Bereich } \\
\text { vermittelt. Andererseits erhielten die Absolvent/innen auch die not- } \\
\text { wendige Sozial-, Beurteilungs- und Reflexionskompetenz, um das } \\
\text { eigene professionelle Handeln in diesen Bereichen kritisch hinter- } \\
\text { fragen zu können. Ein weiteres Ziel des Lehrgangs ist die Bildung } \\
\text { eines Netzwerks, das über die Dauer der Ausbildung hinaus eine } \\
\text { Plattform für Wissenschaftskommunikator/innen in Österreich bietet. }\end{array}$ \\
\hline Zielgruppen & Wissenschafter/innen, Studierende \\
\hline Besucher/Teilnehmerzahlen & 16 \\
\hline Laufzeit & $2005-2006$ \\
\hline
\end{tabular}




\begin{tabular}{l|l}
\hline Projekttitel & sclQ - Wissen für junge Leute \\
\hline Projektträger & sclQ \\
Projekttyp & Magazin \\
Kurzbeschreibung des Projekts & $\begin{array}{l}\text { Das Magazin hat sich auf Wissenschaftskommunikation fokussiert. } \\
\text { Ziel ist es, Jugendlichen, vor allem Mädchen, Lust auf Wissenschaft } \\
\text { und Forschung zu machen, stereotypen Bildern über Wissenschaft } \\
\text { und Forschung entgegen zu wirken sowie spezifische Berufsinforma- } \\
\text { tionen zu liefern. sclQ erscheint sechsmal jährlich und wird in einer } \\
\text { Auflage von zwischen 13.000 und 20.000 Stück an Schulen in Ost- } \\
\text { Österreich (Wien, Niederösterreich, Burgenland) verteilt. sclQ ist } \\
\text { Österreichs einziges Jugend-Medium an der Schnittstelle zwischen } \\
\text { Schule und Wissenschaft/Hochschulen. } \\
\text { Das Themenspektrum reicht von Sex, Simulation, Sonnenenergie, } \\
\text { Sicherheit, Film, Liebe, bis hin zur Angst, Haaren und weiteren The- } \\
\text { men, die den Lebenswelten Jugendlicher entsprechen. Das Ressort } \\
\text { Bildung und Karriere stellt schließlich Berufsbilder vor, die besonders } \\
\text { Mädchen ermutigen sollen, bislang nicht-traditionelle (naturwissen- } \\
\text { schaftliche) Studien zu ergreifen. } \\
\text { Jugendliche } \\
\text { Zielgruppen } \\
\text { Besucher/Teilnehmerzahlen } \\
\text { Laufzeit }\end{array}$ \\
\hline
\end{tabular}

\begin{tabular}{l|l}
\hline Projekttitel & $\begin{array}{l}\text { SCOPE II - Konferenz Zukunftspotenziale der } \\
\text { Kulturerbeforschung }\end{array}$ \\
\hline Projektträger & uma information technology \\
Projekttyp & Konferenz \\
Kurzbeschreibung des Projekts & Interdisziplinäre Konferenz über Strategien für das kulturelle Erbe \\
Zielgruppen & Frauen, Unternehmer/innen, Wissenschafter/innen, Studierende \\
Besucher/Teilnehmerzahlen & 113 \\
Laufzeit & $09 / 2006$ \\
\hline
\end{tabular}

\begin{tabular}{l|l}
\hline Projekttitel & Staatspreis Innovation \\
\hline Projektträger & aws - Austria Wirtschaftsservice \\
Projekttyp & Preis \\
Kurzbeschreibung des Projekts & $\begin{array}{l}\text { Österreichs Betriebe sind innovativ, die besten sollen prämiert } \\
\text { werden, um einer breiten Öffentlichkeit bewusst zu machen, was } \\
\text { heimische Unternehmer und ihre Mitarbeiter/innen leisten. } \\
\text { Unternehmer/innen }\end{array}$ \\
Besucher/Teilnehmerzahlen & $2004: 480 ; 2005: 582 ; 2006:$ k.A. \\
Laufzeit & $2004-2006$ \\
\hline
\end{tabular}




\begin{tabular}{l|l}
\hline Projekttitel & Staatspreis Multimedia \\
\hline $\begin{array}{l}\text { Projektträger } \\
\text { Krojekttyp }\end{array}$ & ICNM - International Center for New Media \\
Kurzbeschreibung des Projekts & Preis \\
& $\begin{array}{l}\text { Der Staatspreis Multimedia und eBusiness zeichnet herausragende } \\
\text { Produkte, Lösungen und Anwendungen mit besonderem Nutzen und } \\
\text { hohem Geschäftswert aus. Seit 1997 ist der Staatspreis der } \\
\text { Branchen-Benchmark. Er bietet umfassenden Überblick über aktuelle } \\
\text { Trends und Marktchancen und ist eine wichtige Promotionsplattform } \\
\text { für alle Einreicher und Gewinner }\end{array}$ \\
Zielgruppen & Frauen, Unternehmer/innen, Wissenschafter/innen, Studierende \\
Besucher/Teilnehmerzahlen & 2006: rd. 600 (Veranstaltung), 206 Teilnehmer/innen \\
Laufzeit & 2004-2005 \\
\hline
\end{tabular}

\begin{tabular}{l|l}
\hline Projekttitel & Stand des Wissens \\
\hline Projektträger & Plansinn \\
Projekttyp & $\begin{array}{l}\text { Informations- und Diskussionsveranstaltungen } \\
\text { Kurzbeschreibung des Projekts }\end{array}$ \\
& $\begin{array}{l}\text { Junge Wissenschafter/innen traten am „Stand des Wissens“ auf } \\
\text { Wochenmärkten in Wien, Linz, Klagenfurt, Oberwart und Steyr mit } \\
\text { Marktbesucher/innen in Dialoge und Diskussionen über aktuelle } \\
\text { Wissenschaft. } \\
\text { Jugendliche, Kinder, Frauen, Unternehmer/innen, Wissen- } \\
\text { schafter/innen, Studierende } \\
\text { Zielgruppen }\end{array}$ \\
$\begin{array}{l}\text { Besucher/Teilnehmerzahlen } \\
\text { Laufzeit }\end{array}$ & $05 / 2006-10 / 2006$ \\
\hline
\end{tabular}

\begin{tabular}{l|l}
\hline Projekttitel & Tage der jungen Forschung - open space for open minds \\
\hline Projektträger & $\begin{array}{l}\text { communication matters } \\
\text { Projekttyp }\end{array}$ \\
Kurzbeschreibung des Projekts & $\begin{array}{l}\text { Projektziel war es, etwa 100 Schüler/innen der vorletzten Klassen } \\
\text { allgemein bildender höherer Schulen in Wien mit 10 jungen Wissen- } \\
\text { schafter/innen zusammenzubringen, um sich kennenzulernen und } \\
\text { realitätsnah über Forschung zu informieren. Auch wurde offene } \\
\text { Diskussion zwischen Politiker/innen (BMVIT), Journalist/innen und } \\
\text { Schüler/innen unter Leitung von Moderatoren veranstaltet. } \\
\text { Jugendliche, Studierende }\end{array}$ \\
$\begin{array}{l}\text { Zielgruppen } \\
\text { Besucher/Teilnehmerzahlen } \\
\text { Laufzeit }\end{array}$ & $\begin{array}{l}\text { 83 } \\
11 / 2005\end{array}$ \\
\hline
\end{tabular}




\begin{tabular}{l|l}
\hline Projekttitel & technology log \\
\hline Projektträger & $\begin{array}{l}\text { Permalink } \\
\text { Web-basiertes Informations- und Wissensmanagement } \\
\text { Krojekttyp } \\
\text { Kurzbeschreibung des Projekts } \\
\text { Technology log ist eine Extranet (bzw. Intranet)-Plattform für Wissens- } \\
\text { und Informationsaustausch verschiedener Organisationen im Feld der } \\
\text { Forschungs- und Innovationsförderung. } \\
\text { Jugendliche, Kinder, Frauen, Unternehmer/innen, Wissen- } \\
\text { schafter/innen, Studierende } \\
\text { k.A. } \\
\text { Besucher/Teilnehmerzahlen } \\
\text { Laufzeit }\end{array}$ \\
\hline
\end{tabular}

\begin{tabular}{|c|c|}
\hline Projekttitel & $\begin{array}{l}\text { Techpol } 2.0 \\
\text { Awareness - Partizipation - Legitimität }\end{array}$ \\
\hline Projektträger & ITA (Institut für Technikfolgenabschätzung) \\
\hline Projekttyp & Veranstaltungsreihe, Analyse \\
\hline \multirow[t]{2}{*}{ Kurzbeschreibung des Projekts } & $\begin{array}{l}\text { Aktuelle forschungs- oder technologiepolitische Fragestellungen mit } \\
\text { Entscheidungsrelevanz wurden mit Bürger/innen diskutiert. }\end{array}$ \\
\hline & $\begin{array}{l}\text { Das primäre Ziel liegt in der legitimationserhöhenden Bereitstellung } \\
\text { von Input für die (forschungs-)politische Entscheidungsfindung. Das } \\
\text { Projekt hat zum Ziel, aus dem Kanon partizipativer Verfahren und } \\
\text { möglicher Themen jene auszuwählen, die für die spezifische Situation } \\
\text { des Rats für Forschung und Technologieentwicklung am besten } \\
\text { geeignet sind und diese für Österreich zu adaptieren. }\end{array}$ \\
\hline Zielgruppen & $\begin{array}{l}\text { Jugendliche, Frauen, Unternehmer/innen, Wissenschafter/innen, } \\
\text { Studierende }\end{array}$ \\
\hline Besucher/Teilnehmerzahlen & k.A. \\
\hline Laufzeit & 09/2005-06/2006 \\
\hline
\end{tabular}




\begin{tabular}{l|l}
\hline Projekttitel & $\begin{array}{l}\text { Transatlantic Discord. Combating Terrorism and Proliferation, } \\
\text { Preventing Crisis }\end{array}$ \\
\hline Projektträger & $\begin{array}{l}\text { Österreichische Gesellschaft für Politikwissenschaft } \\
\text { Konferenz }\end{array}$ \\
Projekttyp & $\begin{array}{l}\text { Anlässlich der zweitägigen Konferenz wurden transatlantische Ge- } \\
\text { meinsamkeiten und Differenzen in der Wahrnehmung von Sicher- } \\
\text { Kurzbeschreibung des Projekts } \\
\text { heitsbedrohungen, vor allem aber im Hinblick auf deren Bewältigung }\end{array}$ \\
Zielgruppen & k.A. \\
Besucher/Teilnehmerzahlen & 80 \\
Laufzeit & $12 / 2005$ \\
\hline
\end{tabular}

\begin{tabular}{l|l}
\hline Projekttitel & Die WAHR/FALSCH Inc. \\
\hline $\begin{array}{l}\text { Projektträger } \\
\text { Projekttyp } \\
\text { Kurzbeschreibung des Projekts }\end{array}$ & $\begin{array}{l}\text { Science Communications } \\
\text { Ausstellung } \\
\text { Die Wissenschaftsausstellung erkundete, wie Forschung Menschen in } \\
\text { ihrem Alltag berührt: Elf thematische Ausstellungsmodule zu unter- } \\
\text { schiedlichen Themen verließen Museum oder Universität. Die Aus- } \\
\text { stellungs-Module entlang der U1 wurden von einem wissenschaft- } \\
\text { lichen "Rechercheteam" entwickelt und zeigen Arbeiten wie Hörspiele, } \\
\text { Videos, oder Installationen zu Themen wie Doping, Allergien, außer- } \\
\text { irdischem Leben, dem Ende des Erdöls oder der Artenvielfalt von } \\
\text { Pflanzen und Tieren in Europa. Dabei lieferte „die wahr/falsch inc.“ } \\
\text { keine Antworten, weil die Wissenschaft - wie die Kunst - immer nur } \\
\text { neue Fragen stellt. Wesentlich war die Rolle der Besucher/innen, die } \\
\text { mit ihrem Wissen und ihren Fragen Teil der „wahr/falsch inc.“ wurden. }\end{array}$ \\
$\begin{array}{l}\text { Jugendliche, Kinder, Frauen, Unternehmer/innen, Wissenschafter/- } \\
\text { innen, Studierende }\end{array}$ \\
$\begin{array}{l}\text { Zielgruppen } \\
\text { Besucher/Teilnehmerzahlen } \\
\text { Laufzeit }\end{array}$ & $06 / 2006-07 / 2006$
\end{tabular}

\begin{tabular}{l|l}
\hline Projekttitel & ZOOM Science \\
\hline $\begin{array}{l}\text { Projektträger } \\
\text { Projekttyp } \\
\text { Kurzbeschreibung des Projekts }\end{array}$ & $\begin{array}{l}\text { ZOOM Kindermuseum } \\
\text { Ausstellung } \\
\text { Das ZOOM Kindermuseum hat bereits in seinem bisherigen Pro- } \\
\text { gramm - Ausstellungen, ZOOM Lab, Atelier, Kindervorlesungen - } \\
\text { einen Schwerpunkt auf die spielerische Vermittlung von Wissen } \\
\text { gelegt. Ziel war es stets, Kindern schon in sehr frühem Alter Zugänge } \\
\text { zur Forschung zu eröffnen und ihre Neugier an wissenschaftlichen }\end{array}$ \\
$\begin{array}{l}\text { Fragestellungen und Methoden zu fördern. Mit der neuen Initiative } \\
\text { ZOOM Science, die ganz im Zeichen der Wissenschaftsvermittlung } \\
\text { für Kinder steht, sollen die bisherigen Aktivitäten gebündelt, verstärkt } \\
\text { und ausgebaut werden. Um selbständiges Lernen zu unterstützen, } \\
\text { steht außerdem die Weblog-Plattform ZOOMblox zur Verfügung: Hier } \\
\text { können die Kinder selbst Weblogs anlegen oder im ZOOM Science- } \\
\text { Weblog ihre Fragen an Expert/innen stellen. } \\
\text { Kinder } \\
\text { Zielgruppen } \\
\text { Besucher/Teilnehmerzahlen } \\
\text { Laufzeit }\end{array}$ & $\begin{array}{l}\text { 25.700 } \\
09 / 2005-03 / 2006\end{array}$ \\
\hline
\end{tabular}





\section{Anhang II: AdEval: Zu Vorgehensweise und Ergebnissen}

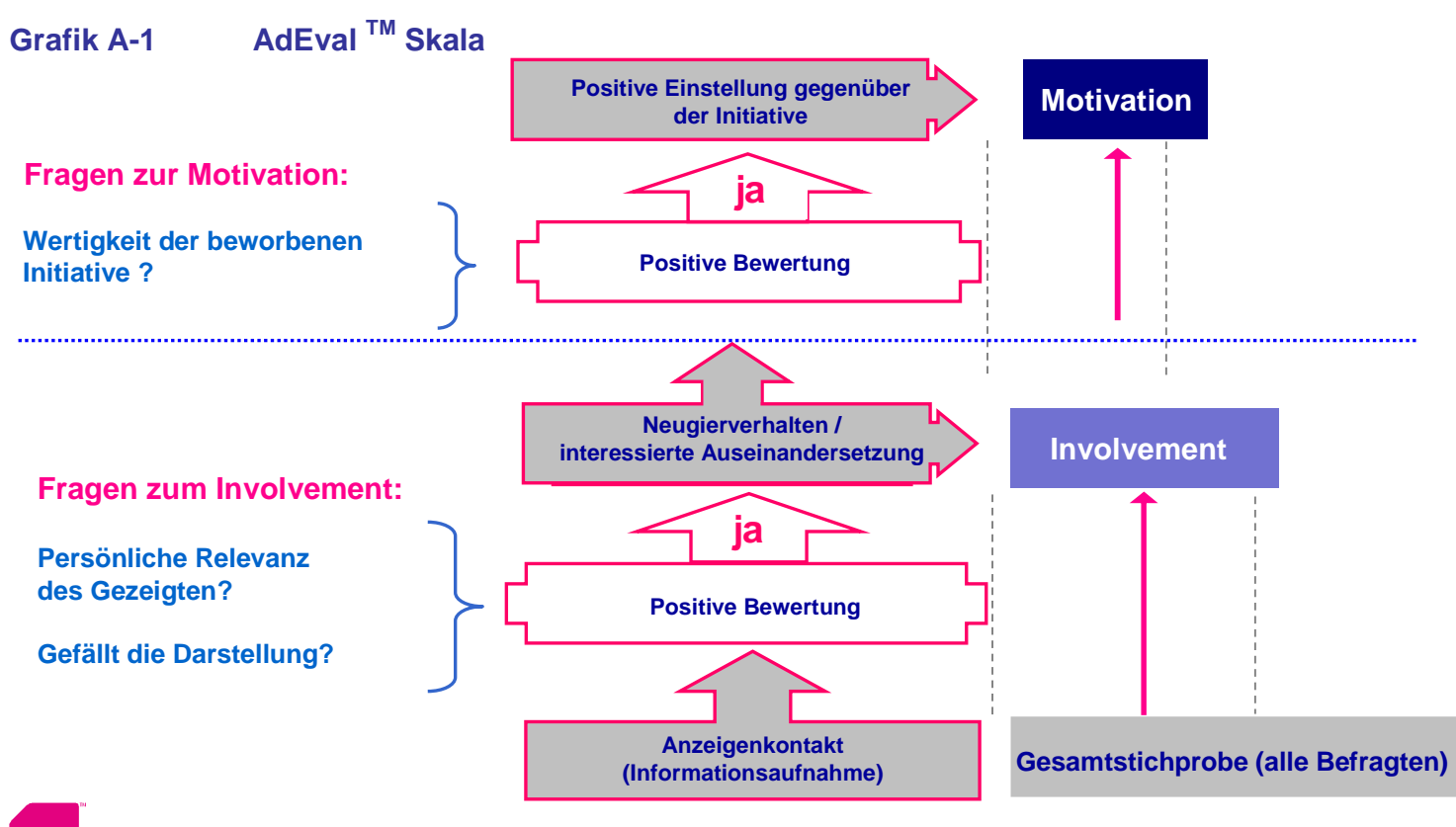

tns infratest

Tabelle A-1: $\quad$ Motivation und Involvement der Kampagne innovatives-oesterreich.at AdEval-Fragestellungen

\begin{tabular}{|c|c|c|c|}
\hline & AdEval-Fragestellungen & 1. Qu. & 4. Qu. \\
\hline \multirow[t]{3}{*}{$\begin{array}{l}\text { Überzeugungs- } \\
\text { leistung } \\
\text { (Involvement) }\end{array}$} & $\begin{array}{l}\text { 1. Hatten Sie beim Ansehen der Anzeige innovatives- } \\
\text { oesterreich den Eindruck, dass sich andere Leute die } \\
\text { Anzeige gern ansehen würden? }\end{array}$ & $63 \%$ & $55 \%$ \\
\hline & $\begin{array}{l}\text { 2. Werbung beachtet man ja normalerweise nicht bewusst oder } \\
\text { gezielt. Aber dennoch gibt es ja die ein oder andere Anzeige, } \\
\text { die man sich gerne anschaut. Wie ist das mit der Anzeige für } \\
\text { die Kampagne innovatives-oesterreich, ist das die Art } \\
\text { Werbung, die Sie sich gerne anschauen würden? }\end{array}$ & $53 \%$ & $46 \%$ \\
\hline & $\begin{array}{l}\text { 3. Hatten Sie bei der Anzeige das Gefühl, dass das, was über } \\
\text { die Kampagne innovatives-oesterreich gezeigt oder gesagt } \\
\text { wurde, sehenswert war? }\end{array}$ & $56 \%$ & $56 \%$ \\
\hline $\begin{array}{l}\text { Motivations- } \\
\text { leistung }\end{array}$ & $\begin{array}{l}\text { 4. Haben Sie jetzt aufgrund dieser Anzeige einen besseren } \\
\text { Eindruck von der Kampagne innovatives-oesterreich? }\end{array}$ & $52 \%$ & $48 \%$ \\
\hline
\end{tabular}

5. Nachfrage „Nein“-Antworten: Und können Sie mir noch einmal sagen, warum Sie keinen besseren Eindruck haben?

$\Rightarrow 24 \%$ bzw. $21 \%$ hatten vorher schon einen guten Eindruck

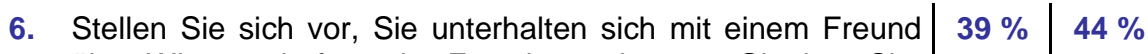
über Wissenschafts- oder Forschungsthemen: Glauben Sie, dass Sie irgendwelche Aussagen, die in der Anzeige über innovatives-oesterreich gemacht wurden, erwähnen würden? Oder dass Sie über bestimmte Eindrücke, die die Anzeige hinterließ, sprechen würden?

\begin{tabular}{l|l}
\hline $\begin{array}{l}\text { Einstellungs- } \\
\text { änderung }\end{array}$ & $\begin{array}{l}\text { Wie würden Sie jetzt - aufgrund dieser Anzeige - Ihre Meinung über die Kampagne } \\
\text { innovatives-oesterreich beschreiben? (viel bessere ... viel schlechtere Meinung) } \\
\end{array}$ \\
\hline $\mathbf{4 9} \%$ bzw. $\mathbf{4 3} \%$ hatten eine bessere Meinung
\end{tabular}

Quelle: TNS Infratest

Prozentangaben bezogen auf Ja-Antworten 